\title{
A new model for quantum dot light emitting-absorbing devices
}

\author{
Dedicated to the memory of Pierre Duclos \\ Hagen Neidhardt \\ Lukas Wilhelm \\ WIAS Berlin, Mohrenstr. 39, 10117 Berlin, Germany \\ E-mail: hagen.neidhardt@wias-berlin.de \\ Valentin A. Zagrebnov \\ Laboratoire d'Analyse, Topologie, Probabilités - UMR 7353 \\ CMI - Technopôle Château-Gombert \\ 39, rue F. Joliot Curie, 13453 Marseille Cedex 13, France \\ E-mail: Valentin.Zagrebnov@latp.univ-mrs.fr
}

July 10, 2021

\begin{abstract}
Motivated by the Jaynes-Cummings (JC) model, we consider here a quantum dot coupled simultaneously to a reservoir of photons and to two electric leads (freefermion reservoirs). This Jaynes-Cummings-Leads (JCL) model makes possible that the fermion current through the dot creates a photon flux, which describes a light-emitting device. The same model is also describe a transformation of the photon flux into current of fermions, i.e. a quantum dot light-absorbing device. The key tool to obtain these results is an abstract Landauer-Büttiker formula.
\end{abstract}

Keywords: Landauer-Büttiker formula, Jaynes-Cummings model, coupling to leads, light emission, solar cells

Mathematics Subject Classification 2000: 47A40, 47A55, 81Q37, 81V80 


\section{Contents}

1 Introduction 2

2 Jaynes-Cummings quantum dot coupled to leads

2.1 Jaynes-Cummings model . . . . . . . . . . . . . . . . . . . 5

2.2 The JCL-model . . . . . . . . . . . . . . . . . . . . . . . 8

2.3 Time reversible symmetric systems . . . . . . . . . . . . . . 11

2.4 Mirror symmetric systems . . . . . . . . . . . . . . . . . . 13

2.5 Spectral properties of $H$ : first part . . . . . . . . . . . . 14

2.6 Spectral representation ........................ 18

2.7 Spectral properties of $H$ : second part . . . . . . . . . 22

3 Landauer-Büttiker formula and applications 26

3.1 Landauer-Büttiker formula . . . . . . . . . . . . . . . . . 26

3.2 Application to the $J C L$-model . . . . . . . . . . . . . . 31

4 Analysis of currents

4.1 Contact induced current . . . . . . . . . . . . . . . . . . . 36

4.2 Photon induced current . . . . . . . . . . . . . 38

5 Electron and photon currents 41

5.1 Electron current . . . . . . . . . . . . . . . . 41

5.1.1 Contact induced electron current . . . . . . . . . . 41

5.1.2 Photon induced electron current . . . . . . . . . . . 42

5.2 Photon current . . . . . . . . . . . . . . . . . 47

5.2.1 Contact induced photon current . . . . . . . . . . . . 47

5.2.2 Photon current ................... 47

\section{Introduction}

The Landauer-Büttiker formula is widely used for the analysis of the steady state current flowing trough a quantum device. It goes back to [18] and [7] and was initially developed based on phenomenological arguments for non-interacting electrons (freefermions). The essential idea was to describe a quantum system as an inner or sample system (dot) with left and right leads attached to it, i.e. free-fermion reservoirs with two different electro-chemical potentials. The goal was to calculate the steady electron current going from one lead through the dot to another one.

It was Landauer and Büttiker who found that this current is directly related to the transmission coefficients of some natural scattering system related to this particle transport problem. The phenomenological approach of Landauer and Büttiker later has been justified in several papers by deriving the formula from fundamental concepts of the Quantum Mechanics, see the series of papers [1, 5, 8, 9, 10, 11, 12, 13, 14] and [19].

Note that this quantum mechanical approach is possible since for the case of freefermion reservoirs the corresponding transport problem reduces to study the Hamiltonian dynamics of extended "one-particle" system. During last decade there has been an 
important progress in rigorous development of the Quantum Statistical Mechanics of Open Systems [2, 3, 4]. This is a many-body approach adapted for interacting systems. It also allows, besides the Hamiltonian [2], to develop a Markovian description of effective microscopic dynamics of the sample system (dot) connected to environment of external reservoirs [3]. Then evolution the sample system is governed by a quantum Master Equation. Although powerful and useful the Markovian approach needs a microscopic Hamiltonian justification, which is a nontrivial problem [3].

In the present paper we follow the one-particle quantum mechanical Hamiltonian approach. Motivated by the quantum optics Jaynes-Cummings (JC) model, we consider here a two-level quantum dot coupled simultaneously to environment of three external reservoirs. The first is the standard JC one-mode photon resonator, which makes the JC quantum dot an open system [16]. Two others are free-fermion reservoirs coupled to the quantum dot. They mimic two electric leads. This new JaynesCummings-Leads ( $J C L-)$ model makes possible that the fermion current through the dot creates a photon flux into the resonator, i.e. it describes a light-emitting device. The same model is also able to describe a transformation of the external photon flux into a current of fermions, which corresponds to a quantum dot light-absorbing device.

The aim of the paper is to analyze the fermion current going through the dot as a function of electro-chemical potentials on leads and the contact with the photon reservoir. Although the latter is the canonical $J C$-interaction, the coupling of the JC model with leads needs certain precautions, if we like to stay in the framework of one-particle quantum mechanical Hamiltonian approach and the scattering theory.

We discuss the construction of our $J C L$-model in Sections 2.2.2.7. For simplicity, we choose for the leads Hamiltonians the one-particle discrete Schrödinger operators with constant one-site (electric) potentials on each of leads. Notice that these Hamiltonians are one band bounded self-adjoint operators. The advantage is that one can easily adjust the leads band spectra positions (and consequently the dot-leads transmission coefficients) shifting them with respect to the two-point quantum dot spectrum by varying the one-site electric potentials (voltage). In Section 2.5 we show that the our model fits into framework of trace-class scattering and in Section 2.7 we verify the important property that the coupled Hamiltonian has no singular continuous spectrum.

Our main tool is an abstract Landauer-Büttiker-type formula applied in Sections 3.1 and 3.2 to the case of the $J C L$-model. Note that this abstract formula allows to calculate not only the electron current but also fluxes for other quantities, such as photon or energy/entropy currents. In particular, we calculate the outgoing flux of photons induced by electric current via leads. This corresponds to a light-emitting device. We also found that pumping the JCL quantum dot by photon flux from resonator may induce current of fermions into leads. This reversing imitates a quantum light-absorbing cell device. These are the main properties of our model and the main application of the Landauer-Büttiker-type formula of Sections 3.1 and 3.2 They are presented in Sections 4 and 5 where we distinguish contact-induced and photon-induced fermion currents.

To describe the results of Sections 4 and 5 note that in our setup the sample Hamiltonian is a two-level quantum dot decoupled from the one-mode resonator. Then the unperturbed Hamiltonian $H_{0}$ describes is a collection of four totally decoupled subsystems: the sample, the resonator and the two leads. The perturbed Hamiltonian $H$ is 
a fully coupled system and the feature of our model is that it is totally (i.e. including the leads) embedded into the external electromagnetic field of resonator. This allows a systematic application of the abstract Landauer-Büttiker-type formula, c.f. Sections 3.1 and 3.2

As we see there is a variety of possibilities to switch on interactions between subsystems, i.e. to produce intermediate Hamiltonians. We distinguish the following two of them:

(a) First to switch on the coupling between sample and resonator: the standard JC model $H_{J C}$, see e.g. [16]. Then to connect it to leads, which gives the Hamiltonian $H_{J C L}:=H$ of the fully coupled system.

(b) First to couple the sample to leads: the corresponding Hamiltonian $H_{S L}$ is a standard "Black Box" $S L$-model for free-fermion current, see [1], [4]. Then to embed it into resonator and to couple the sample with electromagnetic field by the $J C$-interaction. This again produces our $J C L$-model with $H_{J C L}=H$.

Similar to the $S L$-model $\left\{H_{S L}, H_{0}\right\}$, it turns out that the $J C L$-model also fits into the framework of the abstract Landauer-Büttiker formula, and in particular, is a traceclass scattering system $\left\{H_{J C L}=H, H_{S L}\right\}$. The current in the $S L$-model is called the contact-induced current $J_{e l}^{c}$. It was a subject of numerous papers, see e.g. [1, 5], or [4] and references quoted there. Note that the current $J_{e l}$ is due to the difference of electro-chemical potentials between two leads, but it may be zero even if this difference is not null [12, 13].

The fermion current in the $J C L$-model, takes into account the effect of the electron-photon interaction under the assumption that the leads are already coupled. It is called the photon-induced component $J_{e l}^{p h}$ of the total current. Up to our knowledge the present paper is the first, where it is studied rigorously. We show that the total free-fermion current $J$ in the $J C L$-model decomposes into a sum of the contact- and the photon-induced currents: $J_{e l}:=J_{e l}^{c}+J_{e l}^{p h}$. An extremal case is, when the contactinduced current is zero, but the photon-induced component is not, c.f. Section $5.1 \mathrm{In}$ this case the flux of photons $J_{p h}$ out of the quantum dot (sample) is also non-zero, i.e. the dot serves as the light emitting device, c.f. Section 5.2. In general the $J_{p h} \neq 0$ only when the photon-induced component $J_{e l}^{p h} \neq 0$.

In this paper we derive explicit formulas for these currents in the following three cases which are important for the understanding of the $J C L$-model:

(i) The electro-chemical potentials of fermions in the left and right leads are equal. Note that in this case the (contact-induced) current in the $J C L$-model is zero.

(ii) The spectrum of the left and right lead Hamiltonians do not overlap. Again, in this case the contact-induced electron current $J_{e l}^{c}$ of the current in the $J C L$ model is zero, and only the photon-induced electron current $J_{e l}^{p h}$ of the total current is possible.

(iii) The leads are coupled to the Jaynes-Cummings model such that left and right leads interact only by virtue of the photon interaction in the Jaynes-Cummings model. Then the contact-induced electron current $J_{e l}^{c}$ is also zero. 
For these cases we find that the photon induced electron current $J_{\alpha, e l}^{p h}$ entering the left $(\alpha=l)$ or right $(\alpha=r)$ lead is given by

$$
\begin{aligned}
& J_{\alpha, e l}^{p h}=-\sum_{\substack{m, n \in \mathbb{N}_{0} \\
\varkappa \in\{l, r\}}} \frac{\mathfrak{e}}{2 \pi} \int_{\mathbb{R}} d \lambda \widehat{\sigma}_{n_{\varkappa} m_{\alpha}}^{p h}(\lambda) \times \\
& \left(\rho^{p h}(n) f_{F D}\left(\lambda-\mu_{\alpha}-n \omega\right)-\rho^{p h}(m) f_{F D}\left(\lambda-\mu_{\varkappa}-m \omega\right)\right) .
\end{aligned}
$$

where $\widehat{\sigma}_{n_{\varkappa} m_{\alpha}}^{p h}(\lambda) \geq 0$ is a partial scattering cross-section between the left channel with $m$-photons and the $\varkappa$-channel with $n$-photons at energy $\lambda \in \mathbb{R}$. By $\mathfrak{e}>0$ the magnitude of the electron charge is denoted. The photon current is given by

$$
J_{p h}=\sum_{\substack{m, n \in \mathbb{N}_{0} \\ \alpha, \varkappa \in\{l, r\}}}(n-m) \rho^{p h}(m) \frac{1}{2 \pi} \int_{\mathbb{R}} d \lambda f_{F D}\left(\lambda-\mu_{\alpha}-m \omega\right) \widehat{\sigma}_{n_{\varkappa} m_{\alpha}}^{p h}(\lambda) .
$$

Both formulas become simpler if it is assumed that the $J C L$-model is time reversible symmetric. In this case we get

$$
\begin{aligned}
& J_{l, e l}^{p h}=-\sum_{m, n \in \mathbb{N}_{0}} \frac{\mathfrak{e}}{2 \pi} \int_{\mathbb{R}} d \lambda \widehat{\sigma}_{n_{r} m_{l}}^{p h}(\lambda) \times \\
& \left(\rho^{p h}(n) f_{F D}\left(\lambda-\mu_{l}-n \omega\right)-\rho^{p h}(m) f_{F D}\left(\lambda-\mu_{r}-m \omega\right)\right),
\end{aligned}
$$

and

$$
\begin{aligned}
& J_{p h}=\sum_{\substack{m, n \in \mathbb{N}_{0}, n>m \\
\varkappa, \alpha \in\{l, r\}}} \frac{1}{2 \pi} \int_{\mathbb{R}} d \lambda \widehat{\sigma}_{n_{\varkappa} m_{\alpha}}^{p h}(\lambda) \times \\
& (n-m)\left(\rho^{p h}(m) f_{F D}\left(\lambda-\mu_{\alpha}-m \omega\right)-\rho^{p h}(n) f_{F D}\left(\lambda-\mu_{\varkappa}-n \omega\right)\right) .
\end{aligned}
$$

It turns out that choosing the parameters of the model in an suitable manner one gets either a photon emitting or a photon absorbing system. Hence $J C L$-model can be used either as a light emission device or as a light-cell. Proofs of explicit formulas for fermion and photon currents $J_{l, e l}^{p h}, J_{p h}$ is the contents of Sections 4 and 5

Note that the $J C L$-model is called mirror symmetric if (roughly speaking) one can interchange left and right leads and the $J C L$-model remains unchanged. In Section 5 we discuss a surprising example of a mirror symmetric $J C L$-model such that the free-fermion current is zero but the model is photon emitting. This peculiarity is due to a specific choice of the photon-fermion interaction in our model.

\section{Jaynes-Cummings quantum dot coupled to leads}

\subsection{Jaynes-Cummings model}

The starting point for construction of our $J C L$-model is the quantum optics JaynesCummings Hamiltonian $H^{J C}$. Its simplest version is a two-level system (quantum dot) 
with the energy spacing $\varepsilon$, defined by Hamiltonian $h_{S}$ on the Hilbert space $\mathfrak{h}_{S}=\mathbb{C}^{2}$, see e.g. [16]. It is assumed that this system is "open" and interacts with the one-mode $\omega$ photon resonator with Hamiltonian $h^{p h}$.

Since mathematically $h^{p h}$ coincides with quantum harmonic oscillator, the Hilbert space of the resonator is the boson Fock space $\mathfrak{h}^{p h}=\mathfrak{F}_{+}(\mathbb{C})$ over $\mathbb{C}$ and

$$
h^{p h}=\omega b^{*} b .
$$

Here $b^{*}$ and $b$ are verifying the Canonical Commutation Relations $(C C R)$ creation and annihilation operators with domains in $\mathfrak{F}_{+}(\mathbb{C}) \simeq \ell^{2}\left(\mathbb{N}_{0}\right)$. Operator 2.1) is self-adjoint on its domain

$$
\operatorname{dom}\left(h^{p h}\right)=\left\{\left(k_{0}, k_{1}, k_{2}, \ldots\right) \in \ell^{2}\left(\mathbb{N}_{0}\right): \sum_{n \in \mathbb{N}_{0}} n^{2}\left|k_{n}\right|^{2}<\infty\right\} .
$$

Note that canonical basis $\left\{\phi_{n}:=\left(0,0, \ldots, k_{n}=1,0, \ldots\right)\right\}_{n \in \mathbb{N}_{0}}$ in $\ell^{2}\left(\mathbb{N}_{0}\right)$ consists of eigenvectors of operator 2.1]: $h^{p h} \phi_{n}=n \omega \phi_{n}$.

To model the two-level system with the energy spacing $\varepsilon$, one fixes in $\mathbb{C}^{2}$ two ortho-normal vectors $\left\{e_{0}^{S}, e_{1}^{S}\right\}$, for example

$$
e_{0}^{S}:=\left(\begin{array}{l}
0 \\
1
\end{array}\right) \quad \text { and } \quad e_{1}^{S}:=\left(\begin{array}{l}
1 \\
0
\end{array}\right) \text {, }
$$

which are eigenvectors of Hamiltonian $h_{S}$ with eigenvalues $\left\{\lambda_{0}^{S}=0, \lambda_{1}^{S}=\varepsilon\right\}$. To this end we put

$$
h_{S}:=\varepsilon\left(\begin{array}{ll}
1 & 0 \\
0 & 0
\end{array}\right),
$$

and we introduce two ladder operators:

$$
\sigma^{+}:=\left(\begin{array}{ll}
0 & 1 \\
0 & 0
\end{array}\right) \quad, \quad \sigma^{-}:=\left(\begin{array}{ll}
0 & 0 \\
1 & 0
\end{array}\right) .
$$

Then one gets $h_{S}=\varepsilon \sigma^{+} \sigma^{-}$as well as

$$
e_{1}^{S}=\sigma^{+} e_{0}^{S}, e_{0}^{S}=\sigma^{-} e_{1}^{S} \text { and } \sigma^{-} e_{0}^{S}=\left(\begin{array}{l}
0 \\
0
\end{array}\right)
$$

So, $e_{0}^{S}$ is the ground state of Hamiltonian $h_{S}$. Note that non-interacting JaynesCummings Hamiltonian $H_{0}^{J C}$ lives in the space $\mathfrak{H}^{J C}=\mathfrak{h}_{S} \otimes \mathfrak{h}^{p h}=\mathbb{C}^{2} \otimes \mathfrak{F}_{+}(\mathbb{C})$ and it is defined as the matrix operator

$$
H_{0}^{J C}:=h_{S} \otimes I_{\mathfrak{h}^{p h}}+I_{\mathfrak{h}_{S}} \otimes h^{p h} .
$$

Here $I_{\mathfrak{h}}$ ph denotes the unit operator in the Fock space $\mathfrak{h}^{p h}$, whereas $I_{\mathfrak{h}_{S}}$ stays for the unit matrix in the space $\mathfrak{h}_{S}$.

With operators (2.4) the interaction $V_{S b}$ between quantum dot and photons (bosons) in the resonator is defined (in the rotating-wave approximation [16]) by the operator

$$
V_{S b}:=g_{S b}\left(\sigma^{+} \otimes b+\sigma^{-} \otimes b^{*}\right) .
$$


Operators 2.6 and 2.7 define the Jaynes-Cummings model Hamiltonian

$$
H_{J C}:=H_{0}^{J C}+V_{S b}
$$

which is self-adjoint operator on the common domain $\operatorname{dom}\left(H_{0}^{J C}\right) \cap \operatorname{dom}\left(V_{S b}\right)$. The standard interpretation of $H_{J C}$ is that (2.8) describes an "open" two-level system interacting with external one-mode electromagnetic field [16].

Since the one-mode resonator is able to absorb infinitely many bosons this interpretation sounds reasonable, but one can see that the spectrum $\sigma\left(H^{J C}\right)$ of the JaynesCummings model is discrete. To this end note that the so-called number operator

$$
\mathfrak{N}_{J C}:=\sigma^{+} \sigma^{-} \otimes I_{\mathfrak{h}^{p h}}+I_{\mathfrak{h}_{S}} \otimes b^{*} b
$$

commutes with $H_{J C}$. Then, since for any $n \geq 0$

$$
\mathfrak{H}_{n>0}^{J C}:=\left\{\zeta_{0} e_{0}^{S} \otimes \phi_{n}+\zeta_{1} e_{1}^{S} \otimes \phi_{n-1}\right\}_{\zeta_{0,1} \in \mathbb{C}}, \mathfrak{H}_{n=0}^{J C}:=\left\{\zeta_{0} e_{0}^{S} \otimes \phi_{0}\right\}_{\zeta_{0} \in \mathbb{C}},
$$

are eigenspaces of operator $\mathfrak{N}_{J C}$, they reduce $H_{J C}$, i.e. $H_{J C}: \mathfrak{H}_{n}^{J C} \rightarrow \mathfrak{H}_{n}^{J C}$. Note that $\mathfrak{H}^{J C}=\bigoplus_{n \geq 0} \mathfrak{H}_{n}^{J C}$, where each $\mathfrak{H}_{n}^{J C}$ is invariant subspace of operator (2.8). Therefore, it has the representation

$$
H_{J C}=\bigoplus_{n \in \mathbb{N}_{0}} H_{J C}^{(n)}, n>1, H_{J C}^{(0)}=0 .
$$

Here operators $H_{J C}^{(n)}$ are the restrictions of $H_{J C}$, which act in each $\mathfrak{H}_{n}^{J C}$ as

$$
\begin{aligned}
& H_{J C}^{(n)}\left(\zeta_{0} e_{0}^{S} \otimes \phi_{n}+\zeta_{1} e_{1}^{S} \otimes \phi_{n-1}\right)= \\
& {\left[\zeta_{0} n \omega+\zeta_{1} g_{S b} \sqrt{n}\right] e_{0}^{S} \otimes \phi_{n}+\left[\zeta_{1}(\varepsilon+(n-1) \omega)+\zeta_{0} g_{S b} \sqrt{n}\right] e_{1}^{S} \otimes \phi_{n-1} .}
\end{aligned}
$$

Hence, the spectrum $\sigma\left(H_{J C}\right)=\bigcup_{n \geq 0} \sigma\left(H_{J C}^{(n)}\right)$. By virtue of 2.11 the spectrum $\sigma\left(H_{J C}^{(n)}\right)$ is defined for $n \geq 1$ by eigenvalues $E(n)$ of two-by-two matrix $\widehat{H}_{J C}^{(n)}$ acting on the coefficient space $\left\{\zeta_{0}, \zeta_{1}\right\}$ :

$$
\widehat{H}_{J C}^{(n)}\left(\begin{array}{l}
\zeta_{1} \\
\zeta_{0}
\end{array}\right)=\left(\begin{array}{cc}
\varepsilon+(n-1) \omega & g_{S b} \sqrt{n} \\
g_{S b} \sqrt{n} & n \omega
\end{array}\right)\left(\begin{array}{l}
\zeta_{1} \\
\zeta_{0}
\end{array}\right)=E(n)\left(\begin{array}{l}
\zeta_{1} \\
\zeta_{0}
\end{array}\right) .
$$

Then 2.10) and (2.12) imply that the spectrum of the Jaynes-Cummings model Hamiltonian $H_{J C}$ is pure point:

$$
\begin{aligned}
& \sigma\left(H_{J C}\right)=\sigma_{p . p .}\left(H_{J C}\right)= \\
& \{0\} \cup \bigcup_{n \in \mathbb{N}}\left\{n \omega+\frac{1}{2}(\varepsilon-\omega) \pm \sqrt{(\varepsilon-\omega)^{2} / 4+g_{S b}^{2} n}\right\} .
\end{aligned}
$$

This property is evidently persists for any system Hamiltonian $h_{S}$ with discrete spectrum and linear interaction 2.7 with a finite mode photon resonator [16].

We resume the above observations concerning the Jaynes-Cummings model, which is our starting point, by following remarks: 
(a) The standard Hamiltonian (2.8) describes instead of flux only oscillations of photons between resonator and quantum dot, i.e. the system $h_{S}$ is not "open" enough.

(b) Since one our aim is to model a light-emitting device, the system $h_{S}$ needs an external source of energy to pump it into dot, which then be transformed by interaction 2.78 into the outgoing photon current pumping the resonator.

(c) To reach this aim we extend the standard Jaynes-Cummings model to our $J C L$ model by attaching to the quantum dot $h_{S}$ (2.3) two leads, which are (infinite) reservoirs of free fermions. Manipulating with electro-chemical potentials of fermions in these reservoirs we can force one of them to inject fermions in the quantum dot, whereas another one to absorb the fermions out the quantum dot with the same rate. This current of fermions throughout the dot would pump it and produce the photon current according scenario (b).

(d) The most subtle point is to invent a leads-dot interaction $V_{l S}$, which ensures the above mechanism and which is simple enough that one still be able to treat this $J C L$-model using our extension of the Landauer-Büttiker formalism.

\subsection{The JCL-model}

First let us make some general remarks and formulate certain conditions indispensable when one follows the modeling (d).

(1) Note that since the Landauer-Büttiker formalism [13] is essentially a scattering theory on a contact between two subsystems, it is developed only on a "one-particle" level. This allows to study with this formalism only ideal (noninteracting) many-body systems. This condition we impose on many-body fermion systems (electrons) in two leads. Thus, only direct interaction between different components of the system: dot-photons $V_{S b}$ and electron-dot $V_{l S}$ are allowed.

(2) It is well-known that fermion reservoirs are technically simpler to treat then boson ones [13]. Moreover, in the framework of our model it is also very natural since we study electric current although produced by "non-interacting electrons". So, below we use fermions/electrons as synonymous.

(3) In spite of precautions formulated above, the first difficulty to consider an ideal many-body system interacting with quantized electromagnetic field (photons) is induced indirect interaction. If electrons can emit and absorb photons, it is possible for one electron to emit a photon that another electron absorbs, thus creating the indirect photon-mediated electron-electron interaction. This interaction makes impossible to develop the Landauer-Büttiker formula, which requires non-interacting framework.

Assumption 2.1 To solve this difficulty we forbid in our model the photon-mediated interaction. To this end we suppose that every electron (in leads and in dot) interacts 
with its own distinct copy of the electromagnetic field. So, to consider electrons together with its photon fields as non-interacting "composed particles", which allows to apply the Landauer-Büttiker approach. Formally it corresponds to the "one-electron" Hilbert space $\mathfrak{h}^{e l} \otimes \mathfrak{h}^{p h}$, where $\mathfrak{h}^{p h}$ is the Hilbert space of the individual photon field. The fermion description of composed-particles $\mathfrak{h}^{e l} \otimes \mathfrak{h}^{p h}$ corresponds to the antisymmetric Fock space $\mathfrak{F}_{-}\left(\mathfrak{h}^{e l} \otimes \mathfrak{h}^{p h}\right)$.

The composed-particle assumption 2.1 allows us to use the Landauer-Büttiker formalism developed for ideal many-body fermion systems. Now we come closer to the formal description of our JCL-model with two (infinite) leads and a one-mode quantum resonator.

Recall that the Hilbert space of the Jaynes-Cummings Hamiltonian with two energy levels is $\mathfrak{H}^{J C}=\mathbb{C}^{2} \otimes \mathfrak{F}_{+}(\mathbb{C})$. The boson Fock space is constructed from a onedimensional Hilbert space since we consider only photons of a single fixed frequency. We model the electrons in the leads as free fermions living on a discrete semi-infinite lattices. Thus

$$
\mathfrak{h}^{e l}=\ell^{2}(\mathbb{N}) \oplus \mathbb{C}^{2} \oplus \ell^{2}(\mathbb{N})=\mathfrak{h}_{l}^{e l} \oplus \mathfrak{h}_{S} \oplus \mathfrak{h}_{r}^{e l}
$$

is the one-particle Hilbert space for electrons and for the dot. Here, $\mathfrak{h}_{\alpha}^{e l}, \alpha \in\{l, r\}$, are the Hilbert spaces of the left respectively right lead and $\mathfrak{h}_{S}=\mathbb{C}^{2}$ is the Hilbert space of the quantum dot. We denote by

$$
\left\{\delta_{n}^{\alpha}\right\}_{n \in \mathbb{N}}, \quad\left\{\delta_{n}^{S}\right\}_{j=0}^{1}
$$

the canonical basis consisting of individual lattice sites of $\mathfrak{h}_{\alpha}^{e l}, \alpha \in\{l, r\}$, and of $\mathfrak{h}_{S}$, respectively. With the Hilbert space for photons, $\mathfrak{h}^{p h}=\mathfrak{F}_{+}(\mathbb{C}) \simeq \ell^{2}\left(\mathbb{N}_{0}\right)$, we define the Hilbert space of the full system, i.e. quantum dot with leads and with the photon field, as

$$
\mathfrak{H}=\mathfrak{h}^{e l} \otimes \mathfrak{h}^{p h}=\left(\ell^{2}(\mathbb{N}) \oplus \mathbb{C}^{2} \oplus \ell^{2}(\mathbb{N})\right) \otimes \ell^{2}\left(\mathbb{N}_{0}\right)
$$

Remark 2.2 Note that the structure of full space 2.15 takes into account the condition 2.1 and produces composed fermions via the last tensor product. It also manifests that electrons in the dot as well as those in the leads are composed with photons. This makes difference with the picture imposed by the the Jaynes-Cummings model, when only dot is composed with photons:

$$
\mathfrak{H}=\ell^{2}(\mathbb{N}) \oplus \mathbb{C}^{2} \otimes \ell^{2}\left(\mathbb{N}_{0}\right) \oplus \ell^{2}(\mathbb{N}) \quad, \quad \mathfrak{H}^{J C}=\mathbb{C}^{2} \otimes \ell^{2}\left(\mathbb{N}_{0}\right),
$$

see (2.6), 2.7) and (2.8), where $\mathfrak{H}^{J C}=\mathfrak{h}_{S} \otimes \mathfrak{h}^{p h}$. The next step is a choice of interactions between subsystems: dot-resonator-leads.

According to 2.14) the decoupled leads-dot Hamiltonian is the matrix operator

$$
h_{0}^{e l}=\left(\begin{array}{ccc}
h_{l}^{e l} & 0 & 0 \\
0 & h_{S} & 0 \\
0 & 0 & h_{r}^{e l}
\end{array}\right) \text { on } u=\left(\begin{array}{c}
u_{l} \\
u_{S} \\
u_{r}
\end{array}\right),\left\{u_{\alpha} \in \ell^{2}(\mathbb{N})\right\}_{\alpha \in\{l, r\}}, u_{S} \in \mathbb{C}^{2},
$$


where $h_{\alpha}^{e l}=-\Delta^{D}+v_{\alpha}$ with a constant potential bias $v_{\alpha} \in \mathbb{R}, \alpha \in\{l, r\}$, and $h_{S}$ can be any self-adjoint two-by-two matrix with eigenvalues $\left\{\lambda_{0}^{S}, \lambda_{1}^{S}:=\lambda_{0}^{S}+\varepsilon\right\}, \varepsilon>0$, and eigenvectors $\left\{e_{0}^{S}, e_{1}^{S}\right\}$, cf (2.3). Here, $\Delta^{D}$ denotes the discrete Laplacian on $\ell^{2}(\mathbb{N})$ with homogeneous Dirichlet boundary conditions given by

$$
\begin{aligned}
\left(\Delta^{D} f\right)(x) & :=f(x+1)-2 f(x)+f(x-1), \quad x \in \mathbb{N}, \\
\operatorname{dom}\left(\Delta^{D}\right) & :=\left\{f \in \ell^{2}\left(\mathbb{N}_{0}\right): f(0):=0\right\},
\end{aligned}
$$

which is obviously a bounded self-adjoint operator. Notice that $\sigma\left(\Delta^{D}\right)=[0,4]$.

We define the lead-dot interaction for coupling $g_{e l} \in \mathbb{R}$ by the matrix operator acting in 2.14) as

$$
v_{e l}=g_{e l}\left(\begin{array}{ccc}
0 & \left\langle\cdot, \delta_{0}^{S}\right\rangle \delta_{1}^{l} & 0 \\
\left\langle\cdot, \delta_{1}^{l}\right\rangle \delta_{0}^{S} & 0 & \left\langle\cdot, \delta_{1}^{r}\right\rangle \delta_{1}^{S} \\
0 & \left\langle\cdot, \delta_{1}^{S}\right\rangle \delta_{1}^{r} & 0
\end{array}\right),
$$

where non-trivial off-diagonal entries are projection operators in the Hilbert space 2.14 with the scalar product $u, v \mapsto\langle u, v\rangle$ for $u, v \in \mathfrak{h}^{e l}$. Here $\left\{\delta_{0}^{S}, \delta_{1}^{S}\right\}$ is orthonormal basis in $\mathfrak{h}_{S}^{e l}$, which in general may be different from $\left\{e_{0}^{S}, e_{1}^{S}\right\}$. Hence, interaction 2.17 describes quantum tunneling between leads and the dot via contact sites of the leads, which are supports of $\delta_{1}^{l}$ and $\delta_{1}^{r}$.

Then Hamiltonian for the system of interacting leads and dot we define as $h^{e l}:=$ $h_{0}^{e l}+v_{e l}$. Here both $h_{0}^{e l}$ and $h^{e l}$ are bounded self-adjoint operators on $\mathfrak{h}^{e l}$.

Recall that photon Hamiltonian in the one-mode resonator is defined by operator $h^{p h}=\omega b^{*} b$ with domain in the Fock space $\mathfrak{F}_{+}(\mathbb{C}) \simeq \ell^{2}\left(\mathbb{N}_{0}\right)$, 2.1. We denote the canonical basis in $\ell^{2}\left(\mathbb{N}_{0}\right)$ by $\left\{\Upsilon_{n}\right\}_{n \in \mathbb{N}_{0}}$. Then for the spectrum of $h^{p h}$ one obviously gets

$$
\sigma\left(h^{p h}\right)=\sigma_{p p}\left(h^{p h}\right)=\bigcup_{n \in \mathbb{N}_{0}}\{n \omega\} .
$$

We introduce the following decoupled Hamiltonian $H_{0}$, which describes the system when the leads are decoupled from the quantum dot and the electron does not interact with the photon field.

$$
H_{0}:=H_{0}^{e l}+H^{p h}
$$

where

$$
H_{0}^{e l}:=h_{0}^{e l} \otimes I_{\mathfrak{h}^{p h}} \quad \text { and } \quad H^{p h}:=I_{\mathfrak{h}^{e l}} \otimes h^{p h} .
$$

The operator $H_{0}$ is self-adjoint on $\operatorname{dom}\left(H_{0}\right)=\operatorname{dom}\left(I_{\mathfrak{h}}\right.$ el $\left.\otimes h^{p h}\right)$. Recall that $h_{0}^{e l}$ and $h^{p h}$ are bounded self-adjoint operators. Hence $H_{0}^{e l}$ and $H^{e l}$ are semi-bounded from below which yields that $H_{0}$ is semi-bounded from below.

The interaction of the photons and the electrons in the quantum dot is given by the coupling of the dipole moment of the electrons to the electromagnetic field in the rotating wave approximation. Namely,

$$
V_{p h}=g_{p h}\left(\left(\cdot, e_{0}^{S}\right) e_{1}^{S} \otimes b+\left(\cdot, e_{1}^{S}\right) e_{0}^{S} \otimes b^{*}\right)
$$

for some coupling constant $g_{p h} \in \mathbb{R}$. The total Hamiltonian is given by

$$
H:=H^{e l}+H^{p h}+V_{p h}=H_{0}+V_{e l}+V_{p h},
$$


where $H^{e l}:=h^{e l} \otimes I_{\mathfrak{h}^{p h}}$ and $V_{e l}:=v_{e l} \otimes I_{\mathfrak{h}^{p h}}$.

In the following we call $\mathcal{S}=\left\{H, H_{0}\right\}$ the Jaynes-Cummings-leads system, in short $J C L$-model, which we are going to analyze. In particular, we are interested in the electron and photon currents for that system. The analysis will be based on the abstract Landauer-Büttiker formula, cf. [1, 13].

Lemma 2.3 $H$ is bounded from below self-adjoint such that $\operatorname{dom}(H)=\operatorname{dom}\left(H_{0}\right)$.

Proof. Let $c \geq 2$. Then

$$
\left\|b \Upsilon_{n}\right\|^{2} \leq\left\|b^{*} \Upsilon_{n}\right\|^{2}=n+1 \leq c^{-1} n^{2}+c, \quad n \in \mathbb{N}_{0} .
$$

Consider elements $f \in \mathfrak{h}_{S} \otimes \mathfrak{h}^{p h} \cap \operatorname{dom}\left(I_{\mathfrak{h}^{e l}} \otimes h^{p h}\right)$ with

$$
f=\sum_{j, l} \beta_{j l} e_{j} \otimes \Upsilon_{l}, \quad j \in\{0,1\}, \quad l \in \mathbb{N}_{0},
$$

which are dense in $\mathfrak{H}^{J C}:=\mathfrak{h}_{S}^{e l} \otimes h^{p h}$. Then $\|f\|^{2}=\sum_{j, l}\left|\beta_{j l}\right|^{2}$ and $\left\|\left(I_{\mathfrak{h}^{e l}} \otimes b^{*} b\right) f\right\|^{2}=$ $\sum_{j, l=1}\left|\beta_{j l}\right|^{2} l^{2}$. We obtain

$$
\begin{aligned}
& \left\|\left(\left(\cdot, e_{1}^{S}\right) e_{0}^{S} \otimes b\right) f\right\|^{2} \leq \sum_{j, l}\left|\beta_{j l}\right|^{2}\left\|b \Upsilon_{l}\right\|^{2} \leq \\
& \sum_{j, l}\left|\beta_{j l}\right|^{2}\left(c^{-1} l^{2}+c\right)=c^{-1}\left\|\left(I_{\mathfrak{h}}{ }^{e l} \otimes b^{*} b\right) f\right\|^{2}+c\|f\|^{2}
\end{aligned}
$$

Similarly,

$$
\left\|\left(\left(\cdot, e_{1}^{S}\right) e_{0}^{S} \otimes b^{*}\right) f\right\|^{2} \leq c^{-1}\left\|\left(I_{\mathfrak{h}^{e l}} \otimes b^{*} b\right) f\right\|^{2}+c\|f\|^{2} .
$$

If $c \geq 2$ is large enough, then we obtain that $V_{p h}$ is dominated by $H^{p h}$ with relative bound less than one. Hence $H$ is self-adjoint and $\operatorname{dom}\left(H_{0}\right)=\operatorname{dom}(H)$. Since $H_{0}^{e l}$ and $V_{e l}$ are bounded and $H^{p h}$ is self-adjoint and bounded from below, it follows that $H=H_{0}^{e l}+H^{p h}+V_{e l}+V_{p h}$ is bounded from below [17, Thm. V.4.1].

\subsection{Time reversible symmetric systems}

A system described by the Hamiltonian $H$ is called time reversible symmetric if there is a conjugation $\Gamma$ defined on $\mathfrak{H}$ such that $\Gamma H=H \Gamma$. Recall that $\Gamma$ is a conjugation if the conditions $\Gamma^{2}=I$ and $(\Gamma f, \Gamma g)=\overline{(f, g)}, f, g \in \mathfrak{H}$.

Let $\mathfrak{h}_{n}^{p h}, n \in \mathbb{N}_{0}$, the subspace spanned by the eigenvector $\Upsilon_{n}$ in $\mathfrak{h}^{p h}$. We set

$$
\mathfrak{H}_{n_{\alpha}}:=\mathfrak{h}_{\alpha}^{e l} \otimes \mathfrak{h}_{n}^{p h}, \quad n \in \mathbb{N}_{0}, \quad \alpha \in\{l, r\} .
$$

Notice that

$$
\mathfrak{H}=\bigoplus_{n \in \mathbb{N}_{0}, \alpha \in\{l, r\}} \mathfrak{H}_{n_{\alpha}}
$$


Definition 2.4 The $J C L$-model is called time reversible symmetric if there is a conjugation $\Gamma$ acting on $\mathfrak{H}$ such that $H$ and $H_{0}$ are time reversible symmetric and the subspaces $\mathfrak{H}_{n_{\alpha}}, n \in \mathbb{N}_{0}, \alpha \in\{l, r\}$, reduces $\Gamma$.

Example 2.5 Let $\gamma_{\alpha}^{e l}$ and $\gamma_{S}^{e l}$ be conjugations defined by

$$
\gamma_{\alpha}^{e l} f_{\alpha}:=\overline{f_{\alpha}}:=\left\{\overline{f_{\alpha}(k)}\right\}_{k \in \mathbb{N}}, \quad f_{\alpha} \in \mathfrak{h}_{\alpha}^{e l}, \quad \alpha \in\{l, r\},
$$

and

$$
\gamma_{S}^{e l} f_{S}=\gamma_{S}^{e l}\left(\begin{array}{l}
f_{S}(0) \\
f_{S}(1)
\end{array}\right):=\left(\overline{\frac{f_{S}(0)}{f_{S}(1)}}\right)
$$

We set $\gamma^{e l}:=\gamma_{l}^{e l} \oplus \gamma_{S}^{e l} \oplus \gamma_{r}^{e l}$. Further, we set

$$
\gamma^{p h} \psi:=\bar{\psi}=\{\overline{\psi(n)}\}_{n \in \mathbb{N}_{0}}, \quad \psi \in \mathfrak{h}^{p h} .
$$

We set $\Gamma:=\gamma^{e l} \otimes \gamma^{p h}$. One easily checks that $\Gamma$ is a conjugation on $\mathfrak{H}=\mathfrak{h}^{e l} \otimes \mathfrak{h}^{p h}$.

Lemma 2.6 Let $\gamma_{\alpha}^{e l}, \alpha \in\{S, l, r\}$, and $\gamma^{p h}$ be given by Example 2.5

(i) If the conditions $\gamma_{S}^{e l} e_{0}^{S}=e_{0}^{S}$ and $\gamma_{S}^{e l} e_{1}^{S}=e_{1}^{S}$ are satisfied, then $H_{0}$ is time reversible symmetric with respect to $\Gamma$ and, moreover, the subspaces $\mathfrak{H}_{n_{\alpha}}, n \in$ $\mathbb{N}_{0}, \alpha \in\{l, r\}$, reduces $\Gamma$.

(ii) If in addition the conditions $\gamma_{S}^{e l} \delta_{0}^{S}=\delta_{0}^{S}$ and $\gamma_{S}^{e l} \delta_{1}^{S}=\delta_{1}^{S}$ are satisfied, then $J C L$-model is time reversible symmetric.

Proof. (i) Obviously we have

$$
\gamma_{\alpha}^{e l} h_{\alpha}^{e l}=h_{\alpha}^{e l} \gamma_{\alpha}^{e l}, \quad \alpha \in\{l, r\}, \quad \text { and } \quad \gamma^{p h} h^{p h}=h^{p h} \gamma^{p h} .
$$

If $\gamma_{S}^{e l} e_{0}^{S}=e_{0}^{S}$ and $\gamma_{S}^{e l} e_{1}^{S}=e_{1}^{S}$ is satisfied, then $\gamma_{S}^{e l} h_{S}^{e l}=h_{S}^{e l} \gamma_{S}^{e l}$ which yields $\gamma^{e l} h_{0}^{e l}=$ $h_{0}^{e l} \gamma^{e l}$ and, hence, $\Gamma H_{0}=\Gamma H_{0}$. Since $\gamma^{e l} \mathfrak{h}_{\alpha}^{e l}=\mathfrak{h}_{\alpha}^{e l}$ and $\gamma^{p h} \mathfrak{h}^{p h}=\mathfrak{h}^{p h}$ one gets $\Gamma \mathfrak{H}_{n_{\alpha}}=\mathfrak{H}_{n_{\alpha}}$ which shows that $\mathfrak{H}_{n_{\alpha}}$ reduces $\Gamma$.

(ii) Notice that $\gamma_{\alpha}^{e l} \delta_{1}^{\alpha}=\delta_{1}^{\alpha}, \alpha \in\{l, r\}$. If in addition the conditions $\gamma_{S}^{e l} \delta_{0}^{S}=\delta_{0}^{S}$ and $\gamma_{S}^{e l} \delta_{1}^{S}=\delta_{1}^{S}$ are satisfied, then $\gamma^{e l} v_{e l}=v_{e l} \gamma^{e l}$ is valid which yields $\gamma^{e l} h^{e l}=$ $h^{e l} \gamma^{e l}$. Hence $\Gamma H=H \Gamma$. Together with (i) this proves that the $J C L$-model is time reversible symmetric.

Choosing

$$
e_{0}^{S}:=\left(\begin{array}{l}
1 \\
0
\end{array}\right), \quad e_{1}^{S}:=\left(\begin{array}{l}
0 \\
1
\end{array}\right), \quad \delta_{0}^{S}:=\frac{1}{\sqrt{2}}\left(\begin{array}{l}
1 \\
1
\end{array}\right), \quad \delta_{1}^{S}:=\frac{1}{\sqrt{2}}\left(\begin{array}{c}
1 \\
-1
\end{array}\right)
$$

one satisfies the condition $\gamma_{S}^{e l} e_{0}^{S}=e_{0}^{S}$ and $\gamma_{S}^{e l} e_{1}^{S}=e_{1}^{S}$ as well as $\gamma_{S}^{e l} e_{0}^{S}=e_{0}^{S}$ and $\gamma_{S}^{e l} e_{1}^{S}=e_{1}^{S}$. 


\subsection{Mirror symmetric systems}

A unitary operator $U$ acting on $\mathfrak{H}$ is called a mirror symmetry if the conditions

$$
U \mathfrak{H}_{n_{\alpha}}=\mathfrak{H}_{n_{\alpha^{\prime}}}, \quad \alpha, \alpha^{\prime} \in\{l, r\}, \quad \alpha \neq \alpha^{\prime}
$$

are satisfied. In particular, this yields $U \mathfrak{H}^{J C}=\mathfrak{H}^{J C}, \mathfrak{H}^{J C}:=\mathfrak{h}_{S}^{e l} \otimes \mathfrak{h}^{p h}$.

Definition 2.7 The $J C L$-model is called mirror symmetric if there is a mirror symmetry commuting with $H_{0}$ and $H$.

One easily verifies that if $H_{0}$ is mirror symmetric, then

$$
H_{n_{\alpha^{\prime}}} U=U H_{n_{\alpha}}, \quad n \in \mathbb{N}_{0}, \quad \alpha, \alpha^{\prime} \in\{l, r\}, \quad \alpha \neq \alpha^{\prime},
$$

where

$$
H_{n_{\alpha}}:=h_{\alpha}^{e l} \otimes I_{\mathfrak{h}_{n}^{p h}}+I_{\mathfrak{h}_{\alpha}^{e l}} \otimes h_{n}^{p h}=h_{\alpha}^{e l}+n \omega, \quad n \in \mathbb{N}_{0}, \quad \alpha, \alpha^{\prime} \in\{l, r\}, \quad \alpha \neq \alpha^{\prime} .
$$

In particular, this yields that $v_{\alpha}=v_{\alpha^{\prime}}$. Moreover, one gets $U H_{S}=H_{S} U$ where $H_{S}:=h_{S}^{e l} \otimes I_{\mathfrak{h}^{p h}}+I_{\mathfrak{h}^{e l}} \otimes h^{p h}$.

Notice that if $H$ and $H_{0}$ commute with the same mirror symmetry $U$, then also the operator $H_{c}:=h^{e l} \otimes I_{\mathfrak{h}^{p h}}+I_{\mathfrak{h}^{e l}} \otimes h^{p h}$ commutes with $U$, i.e, is mirror symmetric.

Example 2.8 Let $S=\left\{H, H_{0}\right\}$ be the $J C L$-model. Let $v_{l}=v_{r}$ and let $e_{0}^{S}$ and $e_{1}^{S}$ as well as $\delta_{0}^{S}$ and $\delta_{1}^{S}$ be given by (2.23). We set

$$
u_{S}^{e l} e_{0}^{S}:=e_{0}^{S} \quad \text { and } \quad u_{S}^{e l} e_{1}^{S}=-e_{1}^{S}
$$

as well as

$$
u^{p h} \Upsilon_{n}=e^{-i n \pi} \Upsilon_{n}, \quad n \in \mathbb{N}_{0} .
$$

Obviously, $U_{S}:=u_{S}^{e l} \otimes u^{p h}$ defines a unitary operator on $\mathfrak{H}^{J C}$. A straightforward computation shows that

$$
U_{S} H_{S}=H_{S} U_{S} \quad \text { and } \quad U_{S} V_{p h}=V_{p h} U_{S}
$$

Furthermore, we set

$$
u_{r l}^{e l} \delta_{n}^{l}:=\delta_{n}^{r}, \quad \text { and } \quad u_{l r}^{e l} \delta_{n}^{r}=\delta_{n}^{l}, \quad n \in \mathbb{N},
$$

and

$$
u^{e l}:=\left(\begin{array}{ccc}
0 & 0 & u_{l r}^{e l} \\
0 & u_{S}^{e l} & 0 \\
u_{l r}^{e l} & 0 & 0
\end{array}\right)
$$

We have

$$
v_{e l} u^{e l}\left(\begin{array}{c}
f_{l} \\
f_{S} \\
f_{r}
\end{array}\right)=\left(\begin{array}{c}
<f_{S},\left(u_{S}^{e l}\right)^{*} \delta_{0}^{S}>\delta_{1}^{l} \\
<f_{r},\left(u_{l r}^{e l}\right) * \delta_{1}^{l}>\delta_{0}^{S}+<f_{l},\left(u_{r l}^{e l}\right)^{*} \delta_{1}^{r}>\delta_{1}^{S} \\
<f_{S},\left(u_{S}^{e l}\right)^{*} \delta_{1}^{S}>\delta_{1}^{r}
\end{array}\right)
$$


Since $\delta_{0}^{S}:=\frac{1}{\sqrt{2}}\left(e_{0}^{S}+e_{1}^{S}\right)$ and $\delta_{1}^{S}:=\frac{1}{\sqrt{2}}\left(e_{0}^{S}-e_{1}^{S}\right)$ we get from (2.24)

$$
\left(u_{S}^{e l}\right)^{*} \delta_{0}^{S}=\delta_{1}^{S} \quad \text { and } \quad\left(u_{S}^{e l}\right)^{*} \delta_{1}^{S}=\delta_{0}^{S} .
$$

Obviously we have

$$
\left(u_{l r}^{e l}\right)^{*} \delta_{1}^{l}=\delta_{1}^{r} \quad\left(u_{r l}^{e l}\right)^{*} \delta_{1}^{r}=\delta_{1}^{l} .
$$

Inserting (2.29) and (2.30) into 2.28) we find

$$
v_{e l} u^{e l}\left(\begin{array}{c}
f_{l} \\
f_{S} \\
f_{r}
\end{array}\right)=\left(\begin{array}{c}
<f_{S}, \delta_{1}^{S}>\delta_{1}^{l} \\
<f_{r}, \delta_{1}^{r}>\delta_{0}^{S}+<f_{l}, \delta_{1}^{l}>\delta_{1}^{S} \\
<f_{S}, \delta_{0}^{S}>\delta_{1}^{r}
\end{array}\right)
$$

us Further we have

$$
u^{e l} v_{e l}\left(\begin{array}{c}
f_{l} \\
f_{S} \\
f_{r}
\end{array}\right)=\left(\begin{array}{c}
<f_{S}, \delta_{1}^{S}>\delta_{1}^{l} \\
<f_{l}, \delta_{1}^{l}>\delta_{1}^{S}+<f_{r}, \delta_{1}^{r}>\delta_{0}^{S} \\
<f_{s}, \delta_{0}^{S}>\delta_{1}^{r}
\end{array}\right) .
$$

Comparing (2.31) and 2.32) we get $u^{e l} v_{e l}=v_{e l} u^{e l}$. Setting $U:=u^{e l} \otimes u^{p h}$ one immediately proves that $U H_{0}=H_{0} U$ and $U H=H U$. Since $U \mathfrak{H}_{n_{\alpha}}=\mathfrak{H}_{n_{g a^{\prime}}}$ it is satisfied $\mathcal{S}$ is mirror symmetric.

Notice that in addition the Example $2.8 \mathcal{S}$ is time reversible symmetric.

\subsection{Spectral properties of $H$ : first part}

In the following our goal is to apply the Landauer-Büttiker formula to the $J C L$-model. By $\mathfrak{L}_{p}(\mathfrak{H}), 1 \leq p \leq \infty$, we denote in the following the Schatten-v.Neumann ideals.

Proposition 2.9 If $\mathcal{S}=\left\{H, H_{0}\right\}$ is the JCL-model, then $(H+i)^{-1}-\left(H_{0}+i\right)^{-1} \in$ $\mathfrak{L}_{1}(\mathfrak{H})$. In particular, the absolutely continuous parts $H^{a c}$ and $H_{0}^{a c}$ are unitarily equivalent.

Proof. We have

$$
\begin{aligned}
& (H+i)^{-1}-\left(H_{0}+i\right)^{-1}=\left(H_{0}+i\right)^{-1} V(H+i)^{-1}= \\
& \quad\left(H_{0}+i\right)^{-1} V\left(H_{0}+i\right)^{-1}-\left(H_{0}+i\right)^{-1} V\left(H_{0}+i\right)^{-1} V(H+i)^{-1}
\end{aligned}
$$

where $V=H-H_{0}=V_{e l}+V_{p h}$. Taking into account Lemma2.3 it suffices to prove that $\left(H_{0}+i\right)^{-1} V\left(H_{0}+i\right)^{-1} \in \mathfrak{L}_{1}(\mathfrak{H})$. Using the spectral decomposition of $h^{p h}$ with respect to $\mathfrak{h}^{p h}=\bigoplus_{n \in \mathbb{N}_{0}} \mathfrak{h}_{n}^{p h}$, where $\mathfrak{h}_{n}^{p h}$ are the subspaces spanned by $\Upsilon_{n}$, we obtain

$$
\left(H_{0}+i\right)^{-1}=\bigoplus_{n \in \mathbb{N}_{0}}\left(h_{0}^{e l}+n \omega+i\right)^{-1} \otimes I_{\mathfrak{h}_{n}^{p h}} .
$$

We have $\left(H_{0}+i\right)^{-1} V\left(H_{0}+i\right)^{-1}=\left(H_{0}+i\right)^{-1}\left(V_{e l}+V_{p h}\right)\left(H_{0}+i\right)^{-1}$. Since $v_{e l}$ is a finite rank operator we have $\left\|v_{e l}\right\|_{\mathfrak{L}_{1}}<\infty$. Furthermore, $\mathfrak{h}_{n}^{p h}$ is obviously onedimensional for any $n \in \mathbb{N}_{0}$. Hence $\left\|I_{\mathfrak{h}_{n}^{p h}}\right\|_{\mathfrak{L}_{1}}=1$. From (2.33) and $V_{e l}=v_{e l} \otimes I_{\mathfrak{h}^{p h}}$ 
we obtain

$$
\begin{aligned}
\left\|\left(H_{0}+i\right)^{-1} V_{e l}\left(H_{0}+i\right)^{-1}\right\|_{\mathfrak{L}_{1}} & =\sum_{n \in \mathbb{N}_{0}}\left\|\left(h_{0}^{e l}+n \omega+i\right)^{-1} v_{e l}\left(h_{0}^{e l}+n \omega+i\right)^{-1}\right\|_{\mathfrak{L}_{1}} \\
& \leq \sum_{n \in \mathbb{N}_{0}}\left\|\left(h_{0}^{e l}+n \omega+i\right)^{-2}\right\|\left\|v_{e l}\right\|_{\mathfrak{L}_{1}}
\end{aligned}
$$

Since $h_{0}^{e l}$ is bounded we get

$$
\left\|\left(h_{0}^{e l}+n \omega+i\right)^{-1}\right\|=\sup _{\lambda \in \sigma\left(h_{0}^{e l}\right)}\left(\sqrt{(\lambda+n \omega)^{2}+1}\right)^{-1} \leq c(n+1)^{-1}
$$

for some $c>0$. This immediately implies $\left\|\left(H_{0}+i\right)^{-1} V_{e l}\left(H_{0}+i\right)^{-1}\right\|_{\mathfrak{L}_{1}}<\infty$.

We are going to handle $\left(H_{0}+i\right)^{-1} V_{p h}\left(H_{0}+i\right)^{-1}$. Let $p_{n}^{p h}$ be the projection from $\mathfrak{h}^{p h}$ onto $\mathfrak{h}_{n}^{p h}$. We have

$$
\begin{aligned}
& \left(H_{0}+i\right)^{-1}\left(\cdot, e_{0}^{S}\right) e_{1}^{S} \otimes b\left(H_{0}+i\right)^{-1} \\
& \quad=\sum_{m, n \in \mathbb{N}_{0}}\left(h_{0}^{e l}+m \omega+i\right)^{-1}\left(\cdot, e_{0}^{S}\right) e_{1}^{S}\left(h_{0}^{e l}+n \omega+i\right)^{-1} \otimes p_{m}^{p h} b p_{n}^{p h} \\
& \quad=\sum_{n \in \mathbb{N}}\left(h_{0}^{e l}+(n-1) \omega+i\right)^{-1}\left(\cdot, e_{0}^{S}\right) e_{1}^{S}\left(h_{0}^{e l}+n \omega+i\right)^{-1} \otimes \sqrt{n} \Upsilon_{n-1}\left\langle\cdot, \Upsilon_{n}\right\rangle
\end{aligned}
$$

From 2.34 we get

$$
\begin{aligned}
\left.\|\left(h_{0}^{e l}+(n-1) \omega+i\right)^{-1}\left(\cdot, e_{0}^{S}\right) e_{1}^{S}\left(h_{0}^{e l}+n \omega+i\right)^{-1}\right) & \otimes \sqrt{n} \Upsilon_{n}\left\langle\cdot, \Upsilon_{n}\right\rangle \|_{\mathfrak{L}_{1}} \\
& \leq c^{2} \frac{\sqrt{n}}{n(n+1)},
\end{aligned}
$$

$n \in \mathbb{N}$, which yields

$$
\left\|\left(H_{0}+i\right)^{-1}\left(\cdot, e_{0}^{S}\right) e_{1}^{S} \otimes b\left(H_{0}+i\right)^{-1}\right\|_{\mathfrak{L}_{1}} \leq c^{2} \sum_{n \in \mathbb{N}}^{\infty} \frac{\sqrt{n}}{n(n+1)}<\infty .
$$

Since

$\left\|\left(H_{0}+i\right)^{-1}\left(\cdot, e_{1}^{S}\right) e_{0}^{S} \otimes b^{*}\left(H_{0}+i\right)^{-1}\right\|_{\mathfrak{L}_{1}}=\left\|\left(H_{0}+i\right)^{-1}\left(\cdot, e_{0}^{S}\right) e_{1}^{S} \otimes b\left(H_{0}+i\right)^{-1}\right\|_{\mathfrak{L}_{1}}$ one gets $\left(H_{0}+i\right)^{-1} V_{p h}\left(H_{0}+i\right)^{-1} \in \mathfrak{L}_{1}(\mathfrak{H})$ which completes the proof.

Thus, the $J C L$-model $\mathcal{S}=\left\{H, H_{0}\right\}$ is a $\mathfrak{L}_{1}$-scattering system. Let us recall that $h_{\alpha}^{e l}=-\Delta^{D}+v_{\alpha}, \alpha \in\{l, r\}$, on $\mathfrak{h}_{l}^{e l}=\mathfrak{h}_{r}^{e l}=\ell^{2}(\mathbb{N})$.

Lemma 2.10 Let $\alpha \in\{l, r\}$. We have

$$
\sigma\left(h_{\alpha}^{e l}\right)=\sigma_{a c}\left(h_{\alpha}^{e l}\right)=\left[v_{\alpha}, 4+v_{\alpha}\right] .
$$

The normalized generalized eigenfunctions of $h_{\alpha}^{e l}$ are given by

$$
g_{\alpha}(x, \lambda)=\pi^{-\frac{1}{2}}\left(1-\left(-\lambda+2+v_{\alpha}\right)^{2} / 4\right)^{-\frac{1}{4}} \sin \left(\arccos \left(\left(-\lambda+2+v_{\alpha}\right) / 2\right) x\right)
$$

for $x \in \mathbb{N}, \lambda \in\left(v_{\alpha}, 4+v_{\alpha}\right)$. 
Proof. We prove the absolute continuity of the spectrum by showing that

$$
\left\{g_{\alpha}(x, \lambda) \mid \lambda \in(-2,2)\right\}
$$

is a complete set of generalized eigenfunctions. Note that it suffices to prove the lemma for

$$
\left(\left(\Delta^{D}+2\right) f\right)(x)=f(x+1)+f(x-1), \quad f(0)=0 .
$$

The lemma then follows by replacing $\lambda$ with $-\lambda+2+v_{\alpha}$. Let $\lambda \in(-2,2)$ and

$$
g_{\Delta^{D}}(x, \lambda)=\pi^{-\frac{1}{2}}\left(1-\lambda^{2} / 4\right)^{-\frac{1}{4}} \sin (\arccos (\lambda / 2) x)
$$

Note that $g_{\Delta D}(0, \lambda)=0$, whence the boundary condition is satisfied. We substitute $\mu=\arccos (\lambda / 2) \in(0, \pi)$, i.e. $\lambda=2 \cos (\mu)$ and obtain

$$
\sin (\mu(x+1))+\sin (\mu(x-1))=2 \sin (\mu x) \cos (\mu),
$$

whence $g_{\Delta^{D}}(x, \lambda)$ satisfies the eigenvalue equation. It is obvious that $g_{\Delta^{D}}(\cdot, \lambda) \notin$ $\ell^{2}\left(\mathbb{N}_{0}\right)$ for $\lambda \in(-2,2)$. To complete the proof of the lemma, it remains to show the ortho-normality and the completeness. For the ortho-normality, we have to show that

$$
\sum_{x \in \mathbb{N}} g_{\Delta^{D}}(x, \lambda) g_{\Delta^{D}}(x, \nu)=\delta(\lambda-\nu) .
$$

Let $\psi \in C_{0}^{\infty}((-2,2))$. We use the substitution $\mu=\arccos (\nu / 2)$ and the relation

$$
\sin (\arccos (y))=\left(1-y^{2}\right)^{-\frac{1}{2}}
$$

to obtain

$$
\begin{aligned}
& \int_{-2}^{2} \mathrm{~d} \nu \sum_{x \in \mathbb{N}} g_{\Delta^{D}}(x, \lambda) g_{\Delta^{D}}(x, \nu) \psi(\nu) \\
& =2 \pi^{-1} \int_{0}^{\pi} \mathrm{d} \mu \sum_{x \in \mathbb{N}} \frac{\sin (\mu) \sin (\arccos (\lambda / 2) x) \sin (\mu x)}{(\sin (\mu))^{\frac{1}{2}}(\sin (\arccos (\lambda / 2)))^{\frac{1}{2}}} \psi(2 \cos (\mu)) \\
& =(2 \pi)^{-1} \int_{0}^{\pi} \mathrm{d} \mu \sum_{x \in \mathbb{N}} \frac{(\sin (\mu))^{\frac{1}{2}}}{(\sin (\arccos (\lambda / 2)))^{\frac{1}{2}}}\left(e^{i(\arccos (\lambda / 2)-\mu) x}+\right. \\
& \left.e^{-i(\arccos (\lambda / 2)-\mu) x}-e^{i(\arccos (\lambda / 2)+\mu) x}-e^{-i(\arccos (\lambda / 2)+\mu) x}\right) \psi(2 \cos (\mu))
\end{aligned}
$$

Observe that for the Dirichlet kernel

$$
\sum_{x \in \mathbb{N}_{0}}\left(e^{i x y}+e^{-i x y}\right)-1=2 \pi \delta(y),
$$

whence

$$
\begin{aligned}
\int_{-2}^{2} \mathrm{~d} \nu & \sum_{x \in \mathbb{N}} g_{\Delta D}(x, \lambda) g_{\Delta D}(x, \nu) \psi(\nu) \\
= & \int_{0}^{\pi} \mathrm{d} \mu \frac{(\sin (\mu))^{\frac{1}{2}}}{(\sin (\arccos (\lambda / 2)))^{\frac{1}{2}}}(\delta(\arccos (\lambda / 2)-\mu)+ \\
& \delta(\arccos (\lambda / 2)+\mu)) \psi(2 \cos (\mu))=\psi(\lambda) .
\end{aligned}
$$


In the second equality we use that the summand containing $\delta(\arccos (\lambda / 2)+\mu)$ is zero since both $\arccos (\lambda / 2)>0$ and $\mu>0$. Thus, the generalized eigenfunctions are orthonormal. Finally, using once more the substitution $\mu=\arccos (\nu / 2)$, we get

$$
\begin{aligned}
& \int_{-2}^{2} \mathrm{~d} \nu g_{\Delta^{D}}(x, \nu) g_{\Delta^{D}}(y, \nu) \\
& \quad=\int_{-2}^{2} \mathrm{~d} \nu\left(1-(\nu / 2)^{2}\right)^{-\frac{1}{2}} \sin (\arccos (\nu / 2) x) \sin (\arccos (\nu / 2) y) \\
& =2 \pi^{-1} \int_{0}^{\pi} \mathrm{d} \mu(\sin (\mu))^{-1} \sin (\mu) \sin (\mu x) \sin (\mu y) \\
& \quad=\delta_{x y}
\end{aligned}
$$

for $x, y \in \mathbb{N}$, whence the family of generalized eigenfunctions is also complete.

From these two lemmas we obtain the following corollary that gives us the spectral properties of $H_{0}$.

Proposition 2.11 Let $\mathcal{S}=\left\{H, H_{0}\right\}$ be the JCL-model. Then $\sigma\left(H_{0}\right)=\sigma_{a c}\left(H_{0}\right) \cup$ $\sigma_{p p}\left(H_{0}\right)$, where

$$
\sigma_{a c}\left(H_{0}\right)=\bigcup_{n \in \mathbb{N}_{0}}\left[v_{l}+n \omega, v_{l}+4+n \omega\right] \cup\left[v_{r}+n \omega, v_{r}+4+n \omega\right]
$$

and

$$
\sigma_{p p}\left(H_{0}\right)=\bigcup_{n \in \mathbb{N}_{0}}\left\{\lambda_{j}^{S}+n \omega: j=0,1\right\} .
$$

The eigenvectors are given by $\widetilde{g}(m, n)=e_{m}^{S} \otimes \Upsilon_{n}, m=0,1, n \in \mathbb{N}_{0}$. The generalized eigenfunctions are given by $\widetilde{g}_{\alpha}(\cdot, \lambda, n)=g_{\alpha}(\cdot, \lambda-n \omega) \otimes \Upsilon_{n}$ for $\lambda \in \sigma_{a c}\left(H_{0}\right), n \in \mathbb{N}_{0}$, $\alpha \in\{l, r\}$.

Proof. It is well known (see e.g. [15]) that for two self-adjoint operators $A$ and $B$ with $\sigma_{s c}(A)=\sigma_{s c}(B)=\emptyset$, we have $\sigma_{s c}(A \otimes 1+1 \otimes B)=\emptyset$,

$$
\sigma_{a c}(A \otimes 1+1 \otimes B)=\left(\sigma_{a c}(A)+\sigma(B)\right) \cup\left(\sigma(A)+\sigma_{a c}(B)\right)
$$

and

$$
\sigma_{p p}(A \otimes 1+1 \otimes B)=\sigma_{p p}(A)+\sigma_{p p}(B) .
$$

Furthermore, if $\psi_{A}\left(\lambda_{A}\right)$ and $\psi_{B}\left(\lambda_{B}\right)$ are (generalized) eigenfunctions of $A$ and $B$, respectively, then $\psi_{A}\left(\lambda_{A}\right) \otimes \psi_{B}\left(\lambda_{B}\right)$ is a (generalized) eigenfunction of $A \otimes I+I \otimes B$ for the (generalized) eigenvalue $\lambda_{A}+\lambda_{B}$.

The lemma follows now with $A=h_{0}^{e l}$ and $B=h^{p h}$ using Lemmata2.10 and 2.18) and the fact that $h_{S}$ has eigenvectors $\left\{e_{0}^{S}, e_{1}^{S}\right\}$ with eigenvalues $\left\{\lambda_{0}^{S}, \lambda_{1}^{S}=\lambda_{0}^{S}+\varepsilon\right\}$. 


\subsection{Spectral representation}

For the convenience of the reader we define here what we mean under a spectral representation of the absolutely continuous part $K_{0}^{a c}$ of a self-adjoint operator $K_{0}$ on a separable Hilbert space $\mathfrak{K}$. Let $\mathfrak{k}$ be an auxiliary separable Hilbert space. We consider the Hilbert space $L^{2}(\mathbb{R}, d \lambda, \mathfrak{k})$. By $\mathcal{M}$ we define the multiplication operator induced by the independent variable $\lambda$ in $L^{2}(\mathbb{R}, d \lambda, \mathfrak{k})$. Let $\Phi: \mathfrak{K}^{a c}\left(K_{0}\right) \longrightarrow L^{2}(\mathbb{R}, d \lambda, \mathfrak{k})$ be an isometry acting from $\mathfrak{K}^{a c}\left(K_{0}\right)$ into $L^{2}(\mathbb{R}, d \lambda, \mathfrak{k})$ such that $\Phi \operatorname{dom}\left(K_{0}^{a c}\right) \subseteq \operatorname{dom}(\mathcal{M})$ and

$$
\mathcal{M} \Phi f=\Phi K_{0}^{a c} f, \quad f \in \operatorname{dom}\left(K_{0}^{a c}\right) .
$$

Obviously, the orthogonal projection $P:=\Phi \Phi^{*}$ commutes with $\mathcal{M}$ which yields the existence of a measurable family $\{P(\lambda)\}_{\lambda \in \mathbb{R}}$ such that

$$
(P \widehat{f})(\lambda)=P(\lambda) \widehat{f}(\lambda), \quad \widehat{f} \in L^{2}(\mathbb{R}, \lambda, \mathfrak{k}) .
$$

We set $L^{2}(\mathbb{R}, d \lambda, \mathfrak{k}(\lambda)):=P L^{2}(\mathbb{R}, \lambda, \mathfrak{k}), \mathfrak{k}(\lambda):=P(\lambda) \mathfrak{k}$, and call the triplet

$$
\Pi\left(K_{0}^{a c}\right):=\left\{L^{2}(\mathbb{R}, d \lambda, \mathfrak{k}(\lambda)), \mathcal{M}, \Phi\right\}
$$

a spectral representation of $K_{0}^{a c}$. If $\left\{L^{2}(\mathbb{R}, d \lambda, \mathfrak{k}(\lambda)), \mathcal{M}, \Phi\right\}$ is a spectral representation of $K^{a c}$, then $K^{a c}$ is unitarily equivalent $\mathcal{M}_{0}:=\mathcal{M} \uparrow L^{2}(\mathbb{R}, d \lambda, \mathfrak{k}(\lambda))$. Indeed, one has $\Phi K_{0}^{a c} \Phi^{*}=\mathcal{M}_{0}$. The function $\xi_{K_{0}}^{a c}(\lambda):=\operatorname{dom}(\mathfrak{k}(\lambda)), \lambda \in \mathbb{R}$, is called the spectral multiplicity function of $K_{0}^{a c}$. Notice that $0 \leq \xi_{K_{0}}^{a c}(\lambda) \leq \infty$ for $\lambda \in \mathbb{R}$.

For $\alpha \in\{l, r\}$ the generalized eigenfunctions of $h_{\alpha}^{\text {el }}$ define generalized Fourier transforms by $\phi_{\alpha}^{e l}: \mathfrak{h}_{\alpha}^{e l}=\mathfrak{h}_{\alpha}^{e l, a c}\left(h_{\alpha}^{e l}\right) \rightarrow L^{2}\left(\left[v_{\alpha}, v_{\alpha}+4\right]\right)$ and

$$
\left(\phi_{\alpha}^{e l} f_{\alpha}\right)(\lambda)=\sum_{x \in \mathbb{N}_{0}} g_{\alpha}(x, \lambda) f_{\alpha}(x), \quad f_{\alpha} \in \mathfrak{h}_{\alpha}^{e l} .
$$

Setting

$$
\mathfrak{h}_{\alpha}^{e l}(\lambda):= \begin{cases}\mathbb{C} & \lambda \in\left[v_{\alpha}, v_{\alpha}+4\right] \\ 0 & \lambda \in \mathbb{R} \backslash\left[v_{\alpha}, v_{\alpha}+4\right] .\end{cases}
$$

one easily verifies that $\Pi\left(h_{\alpha}^{e l}\right)=\left\{L^{2}\left(\mathbb{R}, d \lambda, \mathfrak{h}_{\alpha}^{e l}(\lambda)\right), \mathcal{M}, \phi_{\alpha}^{e l}\right\}$ is a spectral representation of $h_{\alpha}^{e l}=h_{\alpha}^{e l, a c}, \alpha=l, r$, where we always assumed implicitly that $\left(\phi_{\alpha}^{e l} f_{\alpha}\right)(\lambda)=0$ for $\lambda \in \mathbb{R} \backslash\left[v_{\alpha}, v_{\alpha}+4\right]$. Setting

$$
\mathfrak{h}^{e l}(\lambda):=\underset{\mathfrak{h}_{r}^{e l}(\lambda)}{\mathfrak{h}_{l}^{e l}(\lambda)} \subseteq \mathbb{C}^{2}, \quad \lambda \in \mathbb{R},
$$

and introducing the map

$$
\phi^{e l}: \mathfrak{h}^{e l, a c}\left(h_{0}^{e l}\right)=\underset{\mathfrak{h}_{r}^{e l}}{\stackrel{\mathfrak{h}_{l}^{e l}}{\bigoplus}} \longrightarrow L^{2}\left(\mathbb{R}, d \lambda, \mathfrak{h}^{e l}(\lambda)\right)
$$


defined by

$$
\phi^{e l} f:=\left(\begin{array}{c}
\phi_{l}^{e l} f_{l} \\
\phi_{r}^{e l} f_{r}
\end{array}\right), \quad \text { where } f:=\left(\begin{array}{c}
f_{l} \\
f_{r}
\end{array}\right)
$$

we obtain a spectral representation $\Pi\left(h_{0}^{e l, a c}\right)=\left\{L^{2}\left(\mathbb{R}, d \lambda, \mathfrak{h}^{e l}(\lambda)\right), \mathcal{M}, \phi^{e l}\right\}$ of the absolutely continuous part $h_{0}^{e l, a c}=h_{l}^{e l} \oplus h_{r}^{e l}$ of $h_{0}^{e l}$. One easily verifies that $0 \leq$ $\xi_{h_{0}^{e l}}^{a c}(\lambda) \leq 2$ for $\lambda \in \mathbb{R}$. Introducing

$$
\lambda_{\min }^{e l}:=\min \left\{v_{l}, v_{r}\right\} \quad \text { and } \quad \lambda_{\max }^{e l}:=\max \left\{v_{l}+4, v_{r}+4\right\}
$$

one easily verifies that $\xi_{h_{0}^{a l}}^{a c}(\lambda)=0$ for $\lambda \in \mathbb{R} \backslash\left[\lambda_{\min }^{e l}, \lambda_{\max }^{e l}\right]$.

Notice, if $v_{r}+4 \leq v_{l}$, then

$$
\mathfrak{h}^{e l}(\lambda)= \begin{cases}\mathbb{C}, & \lambda \in\left[v_{r}, v_{r}+4\right] \cup\left[v_{l}, v_{l}+4\right], \\ \{0\}, & \text { otherwise }\end{cases}
$$

which shows that $h_{0}^{e l}$ has simple spectrum. In particular, it holds $\xi_{h_{0}^{e l}}^{a c}(\lambda)=1$ for $\lambda \in\left[v_{r}, v_{r}+4\right] \cup\left[v_{l}, v_{l}+4\right]$ and otherwise $\xi_{h_{0}^{e}}^{a c}(\lambda)=0$.

Let us introduce the Hilbert space $\mathfrak{h}:=l^{2}\left(\mathbb{N}_{0}, \mathbb{C}^{2}\right)=\bigoplus_{n \in \mathbb{N}_{0}} \mathfrak{h}_{n}, \mathfrak{h}_{n}:=\mathbb{C}^{2}$, $n \in \mathbb{N}_{0}$. Regarding $\mathfrak{h}^{e l}(\lambda-n \omega)$ as a subspace of $\mathfrak{h}_{n}$ one regards

$$
\mathfrak{h}(\lambda):=\bigoplus_{n \in \mathbb{N}_{0}} \mathfrak{h}_{n}(\lambda), \quad \mathfrak{h}_{n}(\lambda):=\mathfrak{h}^{e l}(\lambda-n \omega), \quad \lambda \in \mathbb{R},
$$

as a measurable family of subspaces in $\mathfrak{h}$. Notice that $0 \leq \operatorname{dim}(\mathfrak{h}(\lambda))<\infty, \lambda \in \mathbb{R}$. We consider the Hilbert space $L^{2}(\mathbb{R}, d \lambda, \mathfrak{h}(\lambda))$.

Furthermore, we introduce the isometric map $\Phi: \mathfrak{H}\left(H_{0}^{a c}\right) \longrightarrow L^{2}(\mathbb{R}, d \lambda, \mathfrak{h}(\lambda))$ defined by

$$
(\Phi f)(\lambda)=\bigoplus_{n \in \mathbb{N}_{0}}\left(\begin{array}{c}
\left(\phi_{l}^{e l} f_{l}(n)\right)(\lambda-n \omega) \\
\left(\phi_{r}^{e l} f_{r}(n)\right)(\lambda-n \omega)
\end{array}\right), \quad \lambda \in \mathbb{R}
$$

where

$$
\bigoplus_{n \in \mathbb{N}_{0}}\left(\begin{array}{c}
f_{l}(n) \\
f_{r}(n)
\end{array}\right) \in \bigoplus_{n \in \mathbb{N}_{0}} \mathfrak{h}^{e l, a c}\left(h_{0}^{e l}\right) \otimes \mathfrak{h}_{n}^{p h}=\bigoplus_{n \in \mathbb{N}}\left(\begin{array}{c}
\mathfrak{h}_{l}^{e l} \otimes h_{n}^{p h} \\
\oplus \\
\mathfrak{h}_{r}^{e l} \otimes h_{n}^{p h}
\end{array}\right)
$$

where $\mathfrak{h}_{p h}=\bigoplus_{n \in \mathbb{N}_{0}} \mathfrak{h}_{n}^{p h}$ and $\mathfrak{h}_{n}^{p h}$ is the subspace spanned by the eigenvectors $\Upsilon_{n}$ of $h^{p h}$. One easily verifies that $\Phi$ is an isometry acting from $\mathfrak{H}^{a c}\left(H_{0}^{a c}\right)$ onto $L^{2}(\mathbb{R}, d \lambda, \mathfrak{h}(\lambda))$.

Lemma 2.12 The triplet $\left\{L^{2}(\mathbb{R}, d \lambda, \mathfrak{h}(\lambda)), \mathcal{M}, \Phi\right\}$ forms a spectral representation of $H_{0}^{a c}$, that is, $\Pi\left(H_{0}^{a c}\right)=\left\{L^{2}(\mathbb{R}, d \lambda, \mathfrak{h}(\lambda)), \mathcal{M}, \Phi\right\}$ where there is a constant $d \in \mathbb{N}_{0}$ such that $0 \leq \xi_{H_{0}}^{a c}(\lambda) \leq 2 d_{\max }$ for $\lambda \in \mathbb{R}$ where $d_{\max }:=\frac{\lambda_{\max }^{e l}-\lambda_{\min }^{e l}}{\omega}$ and $\lambda_{\max }^{e l}$ and $\lambda_{\min }^{e l}$ are given by $(2.40)$. 
Proof. It remains to show that $\Phi$ transform $H_{0}^{a c}$ into the multiplication operator $\mathcal{M}$. We have

$$
H_{0}^{a c} f=\bigoplus_{n \in \mathbb{N}_{0}}\left(\begin{array}{c}
\left(h_{l}^{e l} f_{l}\right)(n)+n \omega f_{l}(n) \\
\left(h_{r}^{\text {el }} f_{r}\right)(n)+n \omega f_{r}(n)
\end{array}\right)
$$

which yields

$$
\begin{aligned}
& \left(\Phi H_{0}^{a c} f\right)(\lambda) \\
& \quad=\bigoplus_{n \in \mathbb{N}_{0}}\left(\begin{array}{c}
\left(\phi_{l}^{e l}\left(h_{l}^{e l} f_{l}\right)(n)\right)(\lambda-n \omega)+n \omega\left(\phi_{l}^{e l} f_{l}(n)\right)(\lambda-n \omega) \\
\left.\phi_{r}^{\text {el }}\left(h_{r}^{\text {el }} f_{r}\right)(n)\right)(\lambda-n \omega)+n \omega\left(\phi_{r}^{e l} f_{r}(n)\right)(\lambda-n \omega)
\end{array}\right) \\
& \quad=\bigoplus_{n \in \mathbb{N}_{0}}\left(\begin{array}{l}
\lambda\left(\phi_{l}^{e l} f_{l}(n)\right)(\lambda-n \omega) \\
\lambda\left(\phi_{r}^{\text {el }} f_{r}(n)\right)(\lambda-n \omega)
\end{array}\right)=(\mathcal{M} \Phi f)(\lambda), \quad \lambda \in \mathbb{R} .
\end{aligned}
$$

which proves the desired property.

One easily checks that $\mathfrak{h}(\lambda)$ might be only non-trivial if $\lambda-n \omega \in\left[\lambda_{\min }^{e l}, \lambda_{\max }^{e l}\right]$. Hence we get that $\mathfrak{h}(\lambda)$ is non-trivial if the condition

$$
\frac{\lambda-\lambda_{\max }^{e l}}{\omega} \leq n \leq \frac{\lambda-\lambda_{\min }^{e l}}{\omega}
$$

is satisfied. Hence

$$
0 \leq \xi_{H_{0}}^{a c}(\lambda) \leq 2 \operatorname{card}\left\{n \in \mathbb{N}_{0}: \frac{\lambda-\lambda_{\max }^{e l}}{\omega} \leq n \leq \frac{\lambda-\lambda_{\min }^{e l}}{\omega}\right\}, \quad \lambda \in \mathbb{R} .
$$

or

$$
0 \leq \xi_{H_{0}}^{a c}(\lambda) \leq 2 \operatorname{card}\left\{n \in \mathbb{N}_{0}: 0 \leq n \leq \frac{\lambda_{\max }^{e l}-\lambda_{\max }^{e l}}{\omega}\right\}, \quad \lambda \in \mathbb{R} .
$$

Hence $0 \leq \xi_{H_{0}}^{a c}(\lambda) \leq d_{\max }$ for $\lambda \in \mathbb{R}$.

In the following we denote the orthogonal projection from $\mathfrak{h}(\lambda)$ onto $\mathfrak{h}_{n}(\lambda)$ by $P_{n}(\lambda), \lambda \in \mathbb{R}$, cf 2.41). Since $\mathfrak{h}(\lambda)=\bigoplus_{n \in \mathbb{N}_{0}} \mathfrak{h}_{n}(\lambda)$ we have $I_{\mathfrak{h}(\lambda)}=\sum_{n \in \mathbb{N}_{0}} P_{n}(\lambda)$, $\lambda \in \mathbb{R}$. Further, we introduce the subspaces

$$
\mathfrak{h}_{n_{\alpha}}(\lambda):=\mathfrak{h}_{\alpha}^{e l}(\lambda-n \omega), \quad \lambda \in \mathbb{R}, \quad n \in \mathbb{N}_{0} .
$$

Notice that

$$
\mathfrak{h}_{n}(\lambda)=\bigoplus_{\alpha \in\{l, r\}} \mathfrak{h}_{n_{\alpha}}(\lambda), \quad \lambda \in \mathbb{R}, \quad n \in \mathbb{N}_{0}
$$

By $P_{n_{\alpha}}(\lambda)$ we denote the orthogonal projection from $\mathfrak{h}(\lambda)$ onto $\mathfrak{h}_{n_{\alpha}}(\lambda), \lambda \in \mathbb{R}$. Obviously, we have $P_{n}(\lambda)=\sum_{\alpha \in\{l, r\}} P_{n_{\alpha}}(\lambda), \lambda \in \mathbb{R}$.

Example 2.13 In general the direct integral $\Pi\left(H_{0}^{a c}\right)$ can be very complicated, in particular, the structure of $\mathfrak{h}(\lambda)$ given by (2.41) is difficult to analyze. However, there are interesting simple cases: 
(i) Let $v=v_{l}=v_{r}$ and $4 \leq \omega$. In this case we have $\mathfrak{h}^{e l}(\lambda)=\mathbb{C}^{2}$ for $[v, v+4]$ and

$$
\mathfrak{h}(\lambda)= \begin{cases}\mathbb{C}^{2}, & \lambda \in[v+n \omega, v+n \omega+4], \quad n \in \mathbb{N}_{0}, \\ \{0\}, & \text { otherwise. }\end{cases}
$$

(ii) Let $v_{r}=0, v_{l}=4, \omega_{0}=4$. Then

$$
\mathfrak{h}(\lambda)= \begin{cases}\mathfrak{h}_{r}^{e l}(\lambda)=\mathbb{C}, & \lambda \in[0,4), \\ \mathfrak{h}_{l r}^{e l}(\lambda)=\mathbb{C}^{2}, & \lambda \in[4,8), \\ \mathfrak{h}_{r l}^{e l}(\lambda)=\mathbb{C}^{2}, & \lambda \in[8,12), \\ \cdots & \end{cases}
$$

where

$$
\mathfrak{h}_{\alpha \alpha^{\prime}}^{e l}(\lambda)=\underset{\mathfrak{h}_{\alpha^{\prime}}^{e l}(\lambda)}{\mathfrak{h}_{\alpha}^{e l}(\lambda)}, \quad \alpha, \alpha^{\prime} \in\{l, r\}, \quad \alpha \neq \alpha^{\prime}
$$

Hence $\operatorname{dim}(\mathfrak{h}(\lambda))=2$ for $\lambda \geq 4$.

Let $Z$ be a bounded operator acting on $\mathfrak{H}^{a c}\left(H_{0}\right)$ and commuting with $H_{0}^{a c}$. Since $Z$ commutes with $H_{0}^{a c}$ there is a measurable family $\{Z(\lambda)\}_{\lambda \in \mathbb{R}}$ of bounded operators acting on $\mathfrak{h}(\lambda)$ such that $Z$ is unitarily equivalent to the multiplication operator induced by $\{Z(\lambda)\}_{\lambda \in \mathbb{R}}$ in $\Pi\left(H_{0}^{a c}\right)$. We set

$$
Z_{m_{\alpha} n_{\varkappa}}(\lambda):=P_{m_{\alpha}}(\lambda) Z(\lambda) \uparrow \mathfrak{h}_{n_{\varkappa}}(\lambda), \quad \lambda \in \mathbb{R}, \quad m, n \in \mathbb{N}_{0}, \quad \alpha, \varkappa \in\{l, r\} .
$$

Let $Z_{m_{\alpha} n_{\varkappa}}:=P_{m_{\alpha}} Z P_{n_{\varkappa}}$ where $P_{m_{\alpha}}$ is the orthogonal projection from $\mathfrak{H}$ onto $\mathfrak{H}_{m_{\alpha}} \subseteq \mathfrak{H}^{a c}\left(H_{0}\right)$, cf. 2.22). Obviously, the multiplication operator induced $\left\{Z_{m_{\alpha} n_{\varkappa}}(\lambda)\right\}_{\lambda \in \mathbb{R}}$ in $\Pi\left(H_{0}^{a c}\right)$ is unitarily equivalent to $Z_{m_{\alpha} n_{\varkappa}}$.

Since by Lemma $2.12 \mathfrak{h}(\lambda)$ is a finite dimensional space, the operators $Z(\lambda)$ are finite dimensional ones and we can introduce the quantity

$$
\sigma_{m_{\alpha} n_{\varkappa}}(\lambda)=\operatorname{tr}\left(Z_{m_{\alpha} n_{\varkappa}}(\lambda)^{*} Z_{m_{\alpha} n_{\varkappa}}(\lambda)\right), \quad \lambda \in \mathbb{R}, \quad m, n \in \mathbb{N}_{0}, \quad \alpha, \varkappa \in\{l, r\} .
$$

Lemma 2.14 Let $H_{0}$ be the self-adjoint operator defined by 2.19) on $\mathfrak{H}$. Further let $Z$ be a bounded operator on $\mathfrak{H}^{a c}\left(H_{0}\right)$ commuting with $H_{0}^{a c}$

(i) Let $\Gamma$ be a conjugation on $\mathfrak{H}$, cf. Section 2.3 If $\Gamma$ commutes with $H_{0}$ and $P_{n_{\alpha}}$, $n \in \mathbb{N}_{0}, \alpha \in\{l, r\}$ and $\Gamma Z \Gamma=Z^{*}$ holds, then $\sigma_{m_{\alpha} n_{\varkappa}}(\lambda)=\sigma_{n_{\varkappa} m_{\alpha}}(\lambda), \lambda \in \mathbb{R}$.

(ii) Let $U$ be a mirror symmetry on $\mathfrak{H}$. If $U$ commutes with $H_{0}$ and $Z$, then $\sigma_{m_{\alpha} n_{\varkappa}}(\lambda)=\sigma_{m_{\alpha^{\prime}} n_{\varkappa^{\prime}}}(\lambda), \lambda \in \mathbb{R}, m, n \in \mathbb{N}_{0}, \alpha, \alpha^{\prime}, \varkappa, \varkappa^{\prime} \in\{l, r\}, \alpha \neq \alpha^{\prime}, \varkappa \neq \varkappa^{\prime}$.

Proof. (i) Since $\Gamma$ commutes with $H_{0}$ the conjugation $\Gamma$ is reduce by $\mathfrak{H}^{a c}\left(H_{0}\right)$. So without loss of generality we assume that $\Gamma$ acts on $\mathfrak{H}^{a c}\left(H_{0}\right)$. We set $\Gamma_{n_{\alpha}}:=\Gamma\left\lceil\mathfrak{H}_{n_{\alpha}}\right.$. Notice that

$$
\Gamma=\bigoplus_{n \in \mathbb{N}_{0}, \alpha \in\{l, r\}} \Gamma_{n_{\alpha}}
$$


There is a measurable family $\{\Gamma(\lambda)\}_{\lambda \in \mathbb{R}}$ of conjugations such that the multiplication operator induced by $\{\Gamma(\lambda)\}_{\lambda \in \mathbb{R}}$ in $\Pi\left(H_{0}^{a c}\right)$ is unitarily equivalent to $\Gamma$. Moreover, since $\Gamma$ commutes with $P_{n_{\alpha}}$ we get that the multiplication operator induced by the measurable family

$$
\Gamma_{n_{\alpha}}(\lambda):=\Gamma(\lambda)\left\lceil\mathfrak{h}_{n_{\alpha}}(\lambda), \quad \lambda \in \mathbb{R}, \quad m \in \mathbb{N}_{0}, \quad \alpha \in\{l, r\},\right.
$$

is unitarily equivalent to $\Gamma_{n_{\alpha}}$. Using $\Gamma Z \Gamma=Z^{*}$ we get $\Gamma_{m_{\alpha}} Z_{m_{\alpha} n_{\varkappa}} \Gamma_{n_{\varkappa}}=Z_{n_{\varkappa} m_{\alpha}}^{*}$. Hence

$$
\Gamma_{m_{\alpha}}(\lambda) Z_{m_{\alpha} n_{\varkappa}}(\lambda) \Gamma_{n_{\varkappa}}(\lambda)=Z_{n_{\varkappa} m_{\alpha}}(\lambda)^{*}, \quad \lambda \in \mathbb{R} .
$$

If $X$ is trace class operator, then $\operatorname{tr}(\Gamma X \Gamma)=\overline{\operatorname{tr}(X)}$. Using that we find

$$
\frac{\sigma_{m_{\alpha} n_{\varkappa}}(\lambda)=\overline{\operatorname{tr}\left(\Gamma_{n_{\varkappa}}(\lambda) Z_{m_{\alpha} n_{\varkappa}}(\lambda)^{*} Z_{m_{\alpha} n_{\varkappa}}(\lambda) \Gamma_{n_{\varkappa}}(\lambda)\right)}}{\operatorname{tr}\left(\Gamma_{n_{\varkappa}}(\lambda) Z_{m_{\alpha} n_{\varkappa}}(\lambda)^{*} \Gamma_{m_{\alpha}} \Gamma_{m_{\alpha}} Z_{m_{\alpha} n_{\varkappa}}(\lambda) \Gamma_{n_{\varkappa}}(\lambda)\right)}=
$$

From (2.43) we obtain

$$
\sigma_{m_{\alpha} n_{\varkappa}}(\lambda)=\overline{\operatorname{tr}\left(Z_{n_{\varkappa} m_{\alpha}}(\lambda) Z_{n_{\varkappa} m_{\alpha}}(\lambda)^{*}\right)}=\sigma_{n_{\varkappa} m_{\alpha}}(\lambda), \quad \lambda \in \mathbb{R},
$$

which proves (i).

(ii) Again without loss of generality we can assume that $U$ acts only $\mathfrak{H}^{a c}\left(H_{0}\right)$. Since $U$ commutes with $H_{0}$ there is a measurable family $\{U(\lambda)\}_{\lambda \in \mathbb{R}}$ of unitary operators acting on $\mathfrak{h}(\lambda)$ such that the multiplication operator induced by $\{U(\lambda)\}_{\lambda \in \mathbb{R}}$ is unitarily equivalent to $U$. Since $U \mathfrak{H}_{n_{\alpha}}=\mathfrak{H}_{n_{\alpha^{\prime}}}$ we have $U(\lambda) \mathfrak{h}_{n_{\alpha}}(\lambda)=\mathfrak{h}_{n_{\alpha^{\prime}}}(\lambda)$, $\lambda \in \mathbb{R}$. Hence

$$
\begin{gathered}
\sigma_{m_{\alpha} n_{\varkappa}}(\lambda)=\operatorname{tr}\left(U(\lambda) Z_{m_{\alpha} n_{\varkappa}}(\lambda)^{*} Z_{m_{\alpha} n_{\varkappa}}(\lambda) U(\lambda)^{*}\right)= \\
\operatorname{tr}\left(U(\lambda) Z_{m_{\alpha}, n_{\varkappa}}(\lambda)^{*} U(\lambda)^{*} U(\lambda) Z_{m_{\alpha}, n_{\varkappa}}(\lambda) U(\lambda)^{*}\right) .
\end{gathered}
$$

Hence

$$
\sigma_{m_{\alpha} n_{\varkappa}}(\lambda)=\operatorname{tr}\left(P_{n_{\varkappa^{\prime}}} U(\lambda) Z(\lambda)^{*} U(\lambda)^{*} P_{m_{\alpha^{\prime}}}(\lambda) U(\lambda) Z(\lambda) U(\lambda)^{*} P_{n_{\varkappa^{\prime}}}(\lambda)\right) .
$$

Since $U$ commutes with $Z$ we find

$$
\sigma_{m_{\alpha} n_{\varkappa}}(\lambda)=\operatorname{tr}\left(P_{n_{\varkappa^{\prime}}} Z(\lambda)^{*} P_{m_{\alpha^{\prime}}}(\lambda) Z(\lambda) P_{n_{\varkappa^{\prime}}}(\lambda)\right)=\sigma_{m_{\alpha^{\prime}} n_{\varkappa^{\prime}}}(\lambda), \quad \lambda \in \mathbb{R} .
$$

which proves (ii).

\subsection{Spectral properties of $H$ : second part}

Since we have full information on the spectral properties of $H_{0}$ we can use this to show that $H$ has no singular continuous spectrum. Crucial for that is the following lemma: with the help of [6, Cor. IV.15.19], which establishes existence and completeness of wave operators and absence of singular continuous spectrum through a time-falloff method. We cite it as a Lemma for convenience, with slight simplifications that suffice for our purpose. 
Lemma 2.15 ([6, Corollary IV.15.19]) Let $\left\{H_{0}, H\right\}$ be a scattering system and let $\Lambda$ be a closed countable set. Let $F_{+}$and $F_{-}$be two self-adjoint operators such that $F_{+}+F_{-}=P_{H_{0}}^{a c}$ and

$$
s-\lim _{t \rightarrow \infty} e^{\mp i t H_{0}} F_{ \pm} e^{ \pm i t H_{0}}=0 .
$$

If $(H-i)^{-1}-\left(H_{0}-i\right)^{-1} \in \mathfrak{L}_{\infty}(\mathfrak{H}),\left(1-P_{H_{0}}^{a c}\right) \gamma\left(H_{0}\right) \in \mathfrak{L}_{\infty}(\mathfrak{H})$, and

$$
\left|\int_{0}^{ \pm \infty} \mathrm{d} t\left\|\left(\left(H_{0}-i\right)^{-1}-(H-i)^{-1}\right) e^{-i t H_{0}} \gamma\left(H_{0}\right) F_{ \pm}\right\|\right|<\infty
$$

for all $\gamma \in C_{0}^{\infty}(\mathbb{R} \backslash \Lambda)$, then $W_{ \pm}\left(H, H_{0}\right)$ exist and are complete and $\sigma_{s c}(H)=$ $\sigma_{s c}\left(H_{0}\right)=\emptyset$. Furthermore, each eigenvalue of $H$ and $H_{0}$ in $\mathbb{R} \backslash \Lambda$ is of finite multiplicity and these eigenvalues accumulate at most at points of $\Lambda$ or at $\pm \infty$.

We already know that the wave operators exist and are complete since the resolvent difference is trace class. Hence, we need Lemma 2.15 only to prove the following proposition.

Proposition 2.16 The Hamiltonian $H$ defined by 2.21) has no singular continuous spectrum, that is, $\sigma_{s c}(H)=\emptyset$.

Proof. At first we have to construct the operators $F_{ \pm}$. To this end, let $\mathcal{F}: L^{2}(\mathbb{R}) \rightarrow$ $L^{2}(\mathbb{R})$ be the usual Fourier transform, i.e

$$
(\mathcal{F} f)(\mu):=\widehat{f}(\mu):=\frac{1}{\sqrt{2 \pi}} \int_{\mathbb{R}} e^{-i \mu x} f(x) d x, \quad f \in L^{2}(\mathbb{R}, d x), \quad \mu \in \mathbb{R} .
$$

Further, let $\Pi_{ \pm}$be the orthogonal projection onto $L^{2}\left(\mathbb{R}_{ \pm}\right)$in $L^{2}(\mathbb{R})$. We set

$$
F_{ \pm}=\Phi^{*} \mathcal{F} \Pi_{ \pm} \mathcal{F}^{*} \Phi
$$

where $\Phi$ is given by (2.42). We immediately obtain $F_{-}+F_{+}=P_{a c}\left(H_{0}\right)$. We still have to show that

$$
s-\lim _{t \rightarrow \infty}\left\|e^{\mp i t H_{0}} \Phi^{*} \mathcal{F} \Pi_{ \pm} \mathcal{F}^{*} \Phi e^{ \pm i t H_{0}} f\right\|=0
$$

for $f \in \mathfrak{H}^{a c}\left(H_{0}\right)$. We prove the relation only for $F_{+}$since the proof for $F_{-}$is essentially identical. We have

$$
\left(\Pi_{+} \mathcal{F}^{*} \Phi e^{i t H_{0}} f\right)(x)=(2 \pi)^{-\frac{1}{2}} \chi_{\mathbb{R}_{+}}(x) \int_{\mathbb{R}} \mathrm{d} \mu e^{i(x+t) \mu} \widehat{f}(\mu)=\chi_{\mathbb{R}_{+}}(x) \psi(x+t)
$$

with $\psi=\mathcal{F} \widehat{f}$. Now

$$
\begin{aligned}
& \left\|e^{-i t H_{0}} \Phi^{*} \mathcal{F} \Pi_{+} \mathcal{F}^{*} \Phi e^{i t H_{0}} f\right\|^{2}= \\
& \|\| \Pi_{+} \mathcal{F}^{*} \Phi e^{i t H_{0}} f \|^{2}=\int_{\mathbb{R}_{+}} \mathrm{d} x|\psi(x+t)|^{2}=\int_{t}^{\infty} \mathrm{d} x|\psi(x)|^{2} \stackrel{t \rightarrow \infty}{\longrightarrow} 0 .
\end{aligned}
$$


Concerning the compactness condition, we already know that $(H-i)^{-1}-\left(H_{0}-i\right)^{-1} \in$ $\mathfrak{L}_{1}(\mathfrak{H}) \subset \mathfrak{L}_{\infty}(\mathfrak{H})$ from Proposition 2.9. Let

$$
\Lambda=\bigcup_{n \in \mathbb{N}_{0}}\left\{v_{l}+n \omega, v_{r}+n \omega, v_{l}+4+n \omega, v_{r}+4+n \omega\right\},
$$

which is closed and countable. We know from Corollary 2.11 that $H_{0}$ has no singular continuous spectrum and the eigenvalues are of finite multiplicity. It follows that $(1-$ $\left.P_{a c}\left(H_{0}\right)\right) \gamma\left(H_{0}\right)$ is compact for every $\gamma \in C_{0}^{\infty}(\mathbb{R} \backslash \Lambda)$. The remaining assumption of Lemma 2.15 is

$$
\left|\int_{0}^{ \pm \infty} \mathrm{d} t\left\|\left((H-i)^{-1}-\left(H_{0}-i\right)^{-1}\right) \gamma\left(H_{0}\right) e^{-i t H_{0}} F_{ \pm}\right\|\right|<\infty .
$$

If we can prove this, then we immediately obtain that $H$ has no singular continuous spectrum. Now $(H-i)^{-1}-\left(H_{0}-i\right)^{-1}=(H-i)^{-1}\left(V_{e l}+V_{p h}\right)\left(H_{0}-i\right)^{-1}$. But $(H-i)^{-1}$ is bounded,

$$
\operatorname{ran}\left(F_{ \pm}\right) \subset \mathfrak{H}^{a c}\left(H_{0}\right)=\left(\mathfrak{h}_{l}^{e l} \oplus \mathfrak{h}_{r}^{e l}\right) \otimes \mathfrak{h}^{p h},
$$

and $V_{p h} P^{a c}\left(H_{0}\right)=0$. Also, $V_{e l}=v_{e l} \otimes I_{\mathfrak{h}^{p h}}$ and

$$
\operatorname{ker}\left(v_{e l}\right)^{\perp} \subset \mathbb{C} \delta_{1}^{l} \oplus \mathfrak{h}_{S} \oplus \mathbb{C} \delta_{1}^{r} .
$$

Hence, it suffices to prove

$$
\left|\int_{0}^{ \pm \infty} \mathrm{d} t\left\|P_{1}^{\alpha}\left(H_{0}-i\right)^{-1} \gamma\left(H_{0}\right) e^{-i t H_{0}} F_{ \pm}\right\|\right|<\infty
$$

$\alpha \in\{l, r\}$, where $P_{1}^{\alpha}=p_{1}^{\alpha} \otimes I_{\mathfrak{h}^{p h}}$ and $p_{1}^{\alpha}$ is the orthogonal projection onto $\mathfrak{h}_{\alpha}^{e l}$. In the following we treat only the case $F_{+}$. The calculations for $F_{-}$are completely analogous. We use that $\Phi$ maps $H_{0}^{a c}$ into the multiplication operator $\mathcal{M}$ induced by $\lambda$. Hence we get

$$
\begin{aligned}
& \left\|P_{1}^{\alpha} \widetilde{\gamma}\left(H_{0}\right) e^{-i t H_{0}} \Phi^{*} \mathcal{F} f\right\|=\left\|P_{1}^{\alpha} \Phi^{*} \Phi \widetilde{\gamma}\left(H_{0}\right) e^{-i t H_{0}} \Phi^{*} \mathcal{F} f\right\|= \\
& \quad=(2 \pi)^{-\frac{1}{2}}\left(\sum_{n \in \mathbb{N}_{0}}\left|\int_{\delta_{\alpha, n}} \mathrm{~d} \lambda g_{\alpha}(1, \lambda-n \omega) \widetilde{\gamma}(\lambda) \int_{\mathbb{R}_{+}} \mathrm{d} x e^{-i \lambda(x+t)} f(x)\right|^{2}\right)^{\frac{1}{2}}
\end{aligned}
$$

where $\operatorname{supp}(f) \subseteq \mathbb{R}_{+}, \widetilde{\gamma}(\lambda):=(\lambda-i)^{-1} \gamma(\lambda), \lambda \in \mathbb{R}$, and $\delta_{\alpha, n}:=\left[v_{\alpha}+n \omega_{0}, v_{\alpha}+\right.$ $n \omega+4]$. Notice that $\widetilde{\gamma}(\lambda) \in C_{0}^{\infty}(\mathbb{R} \backslash \Lambda)$. We find

$$
\begin{aligned}
& \int_{\delta_{j, n}} \mathrm{~d} \lambda g_{\alpha}(1, \lambda-n \omega) \widetilde{\gamma}(\lambda) \int_{\mathbb{R}_{+}} \mathrm{d} x e^{-i \lambda(x+t)} f(x)= \\
& \int_{v_{\alpha}}^{v_{\alpha}+4} \mathrm{~d} \lambda g_{\alpha}(1, \lambda) \widetilde{\gamma}(\lambda+n \omega) \int_{\mathbb{R}_{+}} \mathrm{d} x e^{-i(\lambda+n \omega)(x+t)} f(x)
\end{aligned}
$$


which yields

$$
\begin{aligned}
& \left\|P_{1}^{\alpha} \Phi^{*} \Phi \widetilde{\gamma}\left(H_{0}\right) e^{-i t H_{0}} \Phi^{*} \mathcal{F} f\right\|= \\
& \quad=(2 \pi)^{-\frac{1}{2}}\left(\sum_{n \in \mathbb{N}_{0}} \mid \int_{v_{\alpha}}^{v_{\alpha}+4} \mathrm{~d} \lambda g_{\alpha}(1, \lambda) \widetilde{\gamma}\left(\lambda+n \omega_{0}\right) \times\right. \\
& \left.\left.\quad \int_{\mathbb{R}_{+}} \mathrm{d} x e^{-i\left(\lambda+n \omega_{0}\right)(x+t)} f(x)\right|^{2}\right)^{\frac{1}{2}} .
\end{aligned}
$$

Since the support of $\gamma(\lambda)$ is compact we get that the sum $\sum_{n \in \mathbb{N}_{0}}$ is finite. Changing the integrals we get

$$
\begin{aligned}
& \int_{\delta_{\alpha, n}} \mathrm{~d} \lambda g_{\alpha}(1, \lambda-n \omega) \widetilde{\gamma}(\lambda) \int_{\mathbb{R}_{+}} \mathrm{d} x e^{-i \lambda(x+t)} f(x)= \\
& \int_{\mathbb{R}_{+}} \mathrm{d} x f(x) e^{-i n \omega_{0}(x+t)} \int_{v_{\alpha}}^{v_{\alpha}+4} \mathrm{~d} \lambda g_{\alpha}(1, \lambda) \widetilde{\gamma}(\lambda+n \omega) e^{-i \lambda(x+t)}
\end{aligned}
$$

Integrating by parts $m$-times we obtain

$$
\begin{aligned}
& \int_{\delta_{\alpha, n}} \mathrm{~d} \lambda g_{\alpha}(1, \lambda-n \omega) \widetilde{\gamma}(\lambda) \int_{\mathbb{R}_{+}} \mathrm{d} x e^{-i \lambda(x+t)} f(x)= \\
& \quad(-i)^{m} \int_{\mathbb{R}_{+}} \mathrm{d} x f(x) \frac{e^{-i n \omega(x+t)}}{(x+t)^{m}} \int_{v_{\alpha}}^{v_{\alpha}+4} \mathrm{~d} \lambda e^{-i \lambda(x+t)} \frac{d^{m}}{d \lambda^{m}}\left(g_{\alpha}(1, \lambda) \widetilde{\gamma}(\lambda+n \omega)\right)
\end{aligned}
$$

Hence

$$
\begin{aligned}
\left|\int_{\delta_{\alpha, n}} \mathrm{~d} \lambda g_{\alpha}(1, \lambda-n \omega) \widetilde{\gamma}(\lambda) \int_{\mathbb{R}_{+}} \mathrm{d} x e^{-i \lambda(x+t)} f(x)\right|^{2} & \\
\leq & C_{n}^{2}\left(\int_{\mathbb{R}_{+}} \mathrm{d} x|f(x)| \frac{1}{(x+t)^{m}}\right)^{2}
\end{aligned}
$$

which yields

$$
\begin{array}{r}
\left|\int_{\delta_{\alpha, n}} \mathrm{~d} \lambda g_{\alpha}(1, \lambda-n \omega) \widetilde{\gamma}(\lambda) \int_{\mathbb{R}_{+}} \mathrm{d} x e^{-i \lambda(x+t)} f(x)\right|^{2} \\
\leq C_{n}^{2} \frac{1}{t^{(2 m-1)}}\|f\|^{2}
\end{array}
$$

for $m \in \mathbb{N}$ where

$$
C_{n}:=\int_{v_{\alpha}}^{v_{\alpha}+4} \mathrm{~d} \lambda \mid \frac{d^{m}}{d \lambda^{m}}\left(g_{\alpha}(1, \lambda) \widetilde{\gamma}(\lambda+n \omega) \mid\right) .
$$

Notice that $C_{n}=0$ for sufficiently large $n \in \mathbb{N}$. Therefore

$$
\left\|P_{1}^{\alpha} \widetilde{\gamma}\left(H_{0}\right) e^{-i t H_{0}} \Phi^{*} \mathcal{F} f\right\| \leq\left(\sum_{n \in \mathbb{N}_{0}} C_{n}^{2}\right)^{1 / 2} \frac{1}{t^{m-1 / 2}}\|f\|, \quad f \in L^{2}\left(\mathbb{R}_{+}, d x\right),
$$

which shows that $\left\|P_{1}^{\alpha} \widetilde{\gamma}\left(H_{0}\right) e^{-i t H_{0}} F_{+}\right\| \in L^{1}\left(\mathbb{R}_{+}, d t\right)$ for $m \geq 2$. 


\section{Landauer-Büttiker formula and applications}

\subsection{Landauer-Büttiker formula}

The abstract Landauer-Büttiker formula can be used to calculate flows through devices. Usually one considers a pair $\mathcal{S}=\left\{K, K_{0}\right\}$ be of self-adjoint operators where the unperturbed Hamiltonian $K_{0}$ describes a totally decoupled system, that means, the inner system is closed and the leads are decoupled from it, while the perturbed Hamiltonian $K$ describes the system where the leads are coupled to the inner system. An important ingredient is system $\mathcal{S}=\left\{K, K_{0}\right\}$ is represents a complete scattering or even a trace class scattering system.

In [1] an abstract Landauer-Büttiker formula was derived in the framework of a trace class scattering theory for semi-bounded self-adjoint operators which allows to reproduce the results of [18] and [7] rigorously. In [13] the results of [1] were generalized to non-semi-bounded operators. Following [1] we consider a trace class scattering system $\mathcal{S}=\left\{K, K_{0}\right\}$. We recall that $\mathcal{S}=\left\{K, K_{0}\right\}$ is called a trace class scattering system if the resolvent difference of $K$ and $K_{0}$ belongs to the trace class. If $\mathcal{S}=\left\{K, K_{0}\right\}$ is a trace class scattering system, then the wave operators $W_{ \pm}\left(K, K_{0}\right)$ exists and are complete. The scattering operator is defined by $S\left(K, K_{0}\right):=W_{+}\left(K, K_{0}\right)^{*} W_{-}\left(K, K_{0}\right)$. The main ingredients besides the trace class scattering system $\mathcal{S}=\left\{K, K_{0}\right\}$ are the density and the charge operators $\rho$ and $Q$, respectively.

The density operator $\rho$ is a non-negative bounded self-adjoint operator commuting with $K_{0}$. The charge $Q$ is a bounded self-adjoint operator commuting also with $K_{0}$. If $K$ has no singular continuous spectrum, then the current related to the density operator $\rho$ and the charge $Q$ is defined by

$$
J_{\rho, Q}^{\mathcal{S}}=-i \operatorname{tr}\left(W_{-}\left(K, K_{0}\right) \rho W_{-}\left(K, K_{0}\right)^{*}[K, Q]\right)
$$

where $[K, Q]$ is the commutator of $K$ and $Q$. In fact, the commutator $[K, Q]$ might be not defined. In this case the regularized definition

$$
J_{\rho, Q}^{\mathcal{S}}=-i \operatorname{tr}\left(W_{-}\left(K, K_{0}\right)\left(I+K_{0}^{2}\right) \rho W_{-}\left(K, K_{0}\right)^{*} \frac{1}{K-i}[K, Q] \frac{1}{K+i}\right)
$$

is used where it is assumed that $\left(I+K_{0}^{2}\right) \rho$ is a bounded operator. Since the condition $(H-i)^{-1}[H, Q](H+i)^{-1} \in \mathfrak{L}_{1}(\mathfrak{H})$ is satisfied the definition (3.2) makes sense. By $\mathfrak{L}_{1}(\mathfrak{H})$ is the ideal of trace class operators is denoted.

Let $K_{0}$ be self-adjoint operator on the separable Hilbert space $\mathfrak{K}$. We call $\rho$ be a density operator for $K_{0}$ if $\rho$ is a bounded non-negative self-adjoint operator commuting with $K_{0}$. Since $\rho$ commutes with $K_{0}$ one gets that $\rho$ leave invariant the subspace $\mathfrak{K}^{a c}\left(K_{0}\right)$. We set

$$
\rho_{a c}:=\rho \uparrow \mathfrak{K}^{a c}\left(K_{0}\right) .
$$

call $\rho_{a c}$ the $a c$-density part of $\rho$.

A bounded self-adjoint operator $Q$ commuting with $K_{0}$ is called a charge. If $Q$ is a charge, then

$$
Q_{a c}:=Q\left\lceil\mathfrak{K}^{a c}\left(K_{0}\right) .\right.
$$


is called its $a c$-charge part.

Let $\Pi\left(K_{0}^{a c}\right)=\left\{L^{2}(\mathbb{R}, d \lambda, \mathfrak{k}(\lambda)), \mathcal{M}, \Phi\right\}$ be a spectral representation of $K_{0}^{a c}$. If $\rho$ is a density operator, then there is a measurable family $\left\{\rho_{a c}(\lambda)\right\}_{\lambda \in \mathbb{R}}$ of bounded self-adjoint operators such that the multiplication operator

$$
\left(\mathcal{M}_{\rho_{a c}} \widehat{f}\right)(\lambda):=\rho_{a c}(\lambda) \widehat{f}(\lambda), \quad \widehat{f} \in \operatorname{dom}\left(M_{\rho_{a c}}\right):=L^{2}(\mathbb{R}, d \lambda, \mathfrak{k}(\lambda)),
$$

is unitarily equivalent to $a c$-part $\rho_{a c}$, that is, $\mathcal{M}_{\rho_{a c}}=\Phi \rho_{a c} \Phi^{*}$. In particular this yields that ess-sup $\operatorname{sef}_{\lambda \in \mathbb{R}}\left\|\rho_{a c}(\lambda)\right\|_{\mathcal{B}(\mathfrak{k}(\lambda)}=\left\|\rho_{a c}\right\|_{\mathcal{B}\left(\mathfrak{K}^{a c}\left(K_{0}\right)\right)}$. In the following we call $\left\{\rho_{a c}(\lambda)\right\}_{\lambda \in \mathbb{R}}$ the density matrix of $\rho_{a c}$.

Similarly, one gets that if $Q$, then there is a measurable family $\left\{Q_{a c}(\lambda)\right\}_{\lambda \in \mathbb{R}}$ of bounded self-adjoint operators such that the multiplication operator

$$
\begin{aligned}
\left(\mathcal{M}_{Q_{a c}} \widehat{f}\right)(\lambda) & :=Q_{a c}(\lambda) \widehat{f}(\lambda), \\
\widehat{f} \in \operatorname{dom}\left(Q_{a c}\right) & :=\left\{f \in L^{2}(\mathbb{R}, d \lambda, \mathfrak{k}(\lambda)): Q_{a c}(\lambda) \widehat{f}(\lambda) \in L^{2}(\mathbb{R}, d \lambda, \mathfrak{k}(\lambda))\right\},
\end{aligned}
$$

is unitarily equivalent to $Q_{a c}$, i.e. $\mathcal{M}_{Q_{a c}}=\Phi Q_{a c} \Phi^{*}$. In particular, one has

$$
\operatorname{ess-sup}_{\lambda \in \mathbb{R}}\left\|Q_{a c}(\lambda)\right\|_{\mathcal{B}(\mathfrak{k}(\lambda))}=\left\|Q_{a c}\right\|_{\mathcal{B}\left(\mathfrak{K}^{a c}\left(K_{0}\right)\right)} .
$$

If $Q$ is a charge, then the family $\left\{Q_{a c}(\lambda)\right\}_{\lambda \in \mathbb{R}}$ is called the charge matrix of the $a c$-part of $Q$.

Let $\mathcal{S}=\left\{K, K_{0}\right\}$ be a trace scattering system. By $\{S(\lambda)\}_{\lambda \in \mathbb{R}}$ we denote the scattering matrix which corresponds to the scattering operator $S\left(K, K_{0}\right)$ with respect to the spectral representation $\Pi\left(K_{0}^{a c}\right)$. The operator $T:=S\left(K, K_{0}\right)-P^{a c}\left(K_{0}\right)$ is called the transmission operator. By $\{T(\lambda)\}_{\lambda \in \mathbb{R}}$ we denote the transmission which is related to the transmission operator. Scattering and transmission matrix are related by $S(\lambda)=T_{\mathfrak{k}(\lambda)}+T(\lambda)$ for a.e. $\lambda \in \mathbb{R}$. Notice that $T(\lambda)$ belongs for to the trace class a.e. $\lambda \in \mathbb{R}$.

Theorem 3.1 ([13, Corollary 2.14]) Let $\mathcal{S}:=\left\{K, K_{0}\right\}$ be a trace class scattering system and let $\{S(\lambda)\}_{\lambda \in \mathbb{R}}$ be the scattering matrix of $\mathcal{S}$ with respect to the spectral representation $\Pi\left(K_{0}^{a c}\right)$. Further let $\rho$ and $Q$ be density and charge operators and let $\left\{\rho_{a c}(\lambda)\right\}_{\lambda \in \mathbb{R}}$ and $\left\{Q_{a c}(\lambda)\right\}_{\lambda \in \mathbb{R}}$ be the density and charge matrices of the ac-parts $\rho_{a c}$ and charge $Q_{a c}$ with respect to $\Pi\left(K_{0}^{a c}\right)$, respectively. If $\left(I+K_{0}^{2}\right) \rho$ is bounded, then the current $J_{\rho, Q}^{\mathcal{S}}$ defined by (3.2) admits the representation

$$
J_{\rho, Q}^{\mathcal{S}}=\frac{1}{2 \pi} \int_{\mathbb{R}} \operatorname{tr}\left(\rho_{a c}(\lambda)\left(Q_{a c}(\lambda)-S^{*}(\lambda) Q_{a c}(\lambda) S(\lambda)\right)\right) \mathrm{d} \lambda
$$

where the integrand on the right hand side and the current $J_{\rho, Q}^{S}$ satisfy the estimate

$$
\begin{aligned}
& \left|\operatorname{tr}\left(\rho_{a c}(\lambda)\left(Q_{a c}(\lambda)-S^{*}(\lambda) Q_{a c}(\lambda) S(\lambda)\right)\right)\right| \leq \\
& 4\|\rho(\lambda)\|_{\mathfrak{L}(\mathfrak{k}(\lambda))}\|T(\lambda)\|_{\mathfrak{L}_{1}(\mathfrak{k}(\lambda))}\|Q(\lambda)\|_{\mathfrak{L}(\mathfrak{k}(\lambda))}
\end{aligned}
$$

for a.e. $\lambda \in \mathbb{R}$ and

$$
\left|J_{\rho, Q}^{\mathcal{S}}\right| \leq C_{0}\left\|(H+i)^{-1}-\left(H_{0}+i\right)^{-1}\right\|_{\mathfrak{L}_{1}(\mathfrak{K})}
$$

where $C_{0}:=\frac{2}{\pi}\left\|\left(1+H_{0}^{2}\right) \rho\right\|_{\mathfrak{L}(\mathfrak{K})}$. 
In applications not every charge $Q$ is a bounded operator. We say the self-adjoint operator $Q$ commuting with $K_{0}$ is a $p$-tempered charge if $Q\left(H_{0}-i\right)^{-p}$ is a bounded operator for $p \in \mathbb{N}_{0}$. As above we can introduce $Q_{a c}:=Q\left\lceil\operatorname{dom}(Q) \cap \mathfrak{K}^{a c}\left(K_{0}\right)\right.$. It turns out that $Q E_{K_{0}}(\Delta)$ is a bounded operator for any bounded Borel set $\Delta$. This yields that the corresponding charge matrix $\left\{Q_{a c}(\lambda)\right\}_{\lambda \in \mathbb{R}}$ is a measurable family of bounded self-adjoint operators such that

$$
\operatorname{ess-sup}_{\lambda \in \mathbb{R}}\left(1+\lambda^{2}\right)^{p / 2}\left\|Q_{a c}(\lambda)\right\|_{\mathfrak{L}(\mathfrak{k}(\lambda))}<\infty .
$$

To generalize the current $J_{\rho, Q}^{S}$ to tempered charges $Q$ one uses the fact that $Q(\Delta):=$ $Q E_{K_{0}}(\Delta)$ is a charge for any bounded Borel set $\Delta$. Hence the current $J_{\rho, Q(\Delta)}^{\mathcal{S}}$ is welldefined by (3.2) for any bounded Borel set $\Delta$. Using Theorem 3.1 one gets that for $p$-tempered charges the limit

$$
J_{\rho, Q}^{\mathcal{S}}:=\lim _{\Delta \rightarrow \mathbb{R}} J_{\rho, Q(\Delta)}^{\mathcal{S}}
$$

exists provided $\left(H_{0}-i\right)^{p+2} \rho$ is a bounded operator. This gives rise for the following corollary.

Corollary 3.2 Let the assumptions of the Theorem 3.1 be satisfied. If for some $p \in \mathbb{N}_{0}$ the operator $\left(H_{0}-i\right)^{p+2} \rho$ is bounded and $Q$ is a p-tempered charge for $K_{0}$, then the current defined by (3.7) admits the representation (3.4) where the right hand side of (3.4) satisfies the estimate (3.5). Moreover, the current $J_{\rho, Q}^{\mathcal{S}}$ can be estimated by

$$
\left|J_{\rho, Q}^{\mathcal{S}}\right| \leq C_{p}\left\|(H+i)^{-1}-\left(H_{0}+i\right)^{-1}\right\|_{\mathfrak{L}_{1}(\mathfrak{K})}
$$

where $C_{p}:=\frac{2}{\pi}\left\|\left(1+H_{0}^{2}\right)^{p+2 / 2} \rho\right\|_{\mathfrak{L}(\mathfrak{K})}\left\|Q\left(I+H_{0}^{2}\right)^{-p / 2}\right\|_{\mathfrak{L}(\mathfrak{K})}$.

At first glance the formula (3.4) is not very similar to the original Landauer-Büttiker formula of [7, 18]. To make the formula more convenient we recall that a standard application example for the Landauer-Büttiker formula is the so-called black-box model, cf. [1]. In this case the Hilbert space $\mathfrak{K}$ is given by

$$
\mathfrak{K}=\mathfrak{K}_{S} \oplus \bigoplus_{j=1}^{N} \mathfrak{K}_{j}, \quad 2 \leq N<\infty .
$$

and $K_{0}$ by

$$
K_{0}=K_{S} \oplus \bigoplus_{j=1}^{N} K_{j}, \quad 2 \leq N<\infty .
$$

The Hilbert space $\mathfrak{K}_{S}$ is called the sample or dot and $K_{S}$ is the sample or dot Hamiltonian. The Hilbert spaces $\mathfrak{K}_{j}$ are called reservoirs or leads and $K_{j}$ are the reservoir or lead Hamiltonians. For simplicity we assume that the reservoir Hamiltonians $K_{j}$ are absolutely continuous and the sample Hamiltonian $K_{S}$ has point spectrum. A typical choice for the density operator is

$$
\rho=f_{S}\left(K_{S}\right) \oplus \bigoplus_{j=1}^{N} f_{j}\left(K_{j}\right),
$$


where $f_{S}(\cdot)$ and $f_{j}(\cdot)$ are non-negative bounded Borel functions, and for the charge

$$
Q=g_{S}\left(H_{s}\right) \oplus \bigoplus_{j=1}^{N} g_{j}\left(H_{j}\right)
$$

where $g_{S}(\cdot)$ and $g_{j}(\cdot)$ a bounded Borel functions. Making this choice the LandauerBüttiker formula (3.4) takes the form

$$
J_{\rho, Q}^{\mathcal{S}}=\frac{1}{2 \pi} \sum_{j, k=1}^{N} \int_{\mathbb{R}}\left(f_{j}(\lambda)-f_{k}(\lambda)\right) g_{j}(\lambda) \sigma_{j k}(\lambda) d \lambda
$$

where

$$
\sigma_{j k}(\lambda):=\operatorname{tr}\left(T_{j k}(\lambda)^{*} T_{j k}(\lambda)\right), \quad j, k=1, \ldots, N, \quad \lambda \in \mathbb{R},
$$

are called the total transmission probability from reservoir $k$ to reservoir $j$, cf. [1]. We call it the cross-section of the scattering process going from channel $k$ to channel $j$ at energy $\lambda \in \mathbb{R} .\left\{T_{j k}(\lambda)\right\}_{\lambda \in \mathbb{R}}$ is called the transmission matrix from channel $k$ to channel $j$ at energy $\lambda \in \mathbb{R}$ with respect to the spectral representation $\Pi\left(K_{0}^{a c}\right)$. We note that $\left\{T_{j k}(\lambda)\right\}_{\lambda \in \mathbb{R}}$ corresponds to the transmission operator

$$
T_{j k}:=P_{j} T\left(K, K_{0}\right) P_{k}, \quad T\left(K, K_{0}\right):=S\left(K, K_{0}\right)-P^{a c}\left(K_{0}\right),
$$

acting from the reservoir $k$ to reservoir $j$ where $T\left(K, K_{0}\right)$ is called the transmission operator. Let $\{T(\lambda)\}_{\lambda \in \mathbb{R}}$ be the transmission matrix. Following [1] the current $J_{\rho, Q}^{\mathcal{S}}$ given by 3.13 is directed from the reservoirs into the sample.

The quantity $\|T(\lambda)\|_{\mathfrak{L}_{2}}=\operatorname{tr}\left(T(\lambda)^{*} T(\lambda)\right)$ is well-defined and is called the crosssection of the scattering system $\mathcal{S}$ at energy $\lambda \in \mathbb{R}$. Notice that

$$
\sigma(\lambda)=\|T(\lambda)\|_{\mathfrak{L}_{2}}=\operatorname{tr}\left(T(\lambda)^{*} T(\lambda)\right)=\sum_{j, k=1}^{N} \sigma_{j k}(\lambda) . \quad \lambda \in \mathbb{R},
$$

We point out that the channel cross-sections $\sigma_{j k}(\lambda)$ admit the property

$$
\sum_{j=1}^{N} \sigma_{j k}(\lambda)=\sum_{j=1}^{N} \sigma_{k j}(\lambda), \quad \lambda \in \mathbb{R}
$$

which is a consequence of the unitarity of the scattering matrix. Moreover, if there is a conjugation $J$ such that $K J=J K$ and $K_{0} J=J K_{0}$ holds, that is, if the scattering system $\mathcal{S}$ is time reversible symmetric, then we have even more, namely, it holds

$$
\sigma_{j k}(\lambda)=\sigma_{k j}(\lambda), \quad \lambda \in \mathbb{R} .
$$

Usually the Landauer-Büttiker formula 3.13 is used to calculated the electron current entering the reservoir $j$ from the sample. In this case one has to choose $Q:=$ $Q_{j}^{e l}:=-\mathfrak{e} P_{j}$ where $P_{j}$ is the orthogonal projection form $\mathfrak{K}$ onto $\mathfrak{K}_{j}$ and $\mathfrak{e}>0$ is the magnitude of the elementary charge. This is equivalent to choose $g_{j}(\lambda)=-\mathfrak{e}$ and 
$g_{k}(\lambda)=0$ for $k \neq j, \lambda \in \mathbb{R}$. Doing so we get the Landauer-Büttiker formula simplifies to

$$
J_{\rho, Q_{j}^{e l}}^{\mathcal{S}}=-\frac{\mathfrak{e}}{2 \pi} \sum_{k=1}^{N} \int_{\mathbb{R}}\left(f_{j}(\lambda)-f_{k}(\lambda)\right) \sigma_{j k}(\lambda) d \lambda .
$$

To restore the original Landauer-Büttiker formula one sets

$$
f_{j}(\lambda)=f\left(\lambda-\mu_{j}\right), \quad \lambda \in \mathbb{R},
$$

where $\mu_{j}$ is the chemical potential of the reservoir $\mathfrak{K}_{j}$ and $f(\cdot)$ is a bounded nonnegative Borel function called the distribution function. This gives to the formula

$$
J_{\rho, Q_{j}^{e l}}^{\mathcal{S}}=-\frac{\mathfrak{e}}{2 \pi} \sum_{k=1}^{N} \int_{\mathbb{R}}\left(f\left(\lambda-\mu_{j}\right)-f\left(\lambda-\mu_{k}\right)\right) \sigma_{j k}(\lambda) d \lambda .
$$

In particular, if we choose one

$$
f(\lambda):=f_{F D}(\lambda):=\frac{1}{1+e^{\beta \lambda}}, \quad \beta>0, \quad \lambda \in \mathbb{R},
$$

where $f_{F D}(\cdot)$ is the Fermi-Dirac distribution function, and inserting (3.21) into (3.20) we obtain

$$
J_{\rho, Q_{j}^{e l}}^{\mathcal{S}}=-\frac{\mathfrak{e}}{2 \pi} \sum_{k=1}^{N} \int_{\mathbb{R}}\left(f_{F D}\left(\lambda-\mu_{j}\right)-f_{F D}\left(\lambda-\mu_{k}\right)\right) \sigma_{j k}(\lambda) d \lambda .
$$

If we have only two reservoirs, then they are usually denoted by $l$ (left) and $r$ (right). Let $j=l$ and $k=r$. Then

$$
J_{\rho, Q_{l}^{e l}}^{\mathcal{S}}=-\frac{\mathfrak{e}}{2 \pi} \int_{\mathbb{R}}\left(f_{F D}\left(\lambda-\mu_{l}\right)-f_{F D}\left(\lambda-\mu_{r}\right)\right) \sigma_{l r}(\lambda) d \lambda .
$$

One easily checks that $J_{\rho, Q_{l}}^{S} \leq 0$ if $\mu_{l} \geq \mu_{r}$. That means, the current is leaving the left reservoir and is entering the right one which is accordance with physical intuition.

Example 3.3 Notice that $s_{c}:=\left\{h^{e l}, h_{0}^{e l}\right\}$ is a $\mathfrak{L}_{1}$ scattering system. The Hamiltonian $h^{e l}$ takes into account the effect of coupling of reservoirs or leads $\mathfrak{h}_{l}:=l^{2}(\mathbb{N})$ and $\mathfrak{h}_{r}:=l^{2}(\mathbb{N})$ to the sample $\mathfrak{h}_{S}=\mathbb{C}^{2}$ which is also called the quantum dot. The leads Hamiltonian are given by $h_{\alpha}^{e l}=-\Delta^{D}+v_{\alpha}, \alpha=l, r$. The sample or quantum dot Hamiltonian is given by $h_{S}^{e l}$. The wave operators are given by

$$
w_{ \pm}\left(h^{e l}, h_{0}^{e l}\right):=s-\lim _{t \rightarrow \infty} e^{i t h^{e l}} e^{-i t h_{0}^{e l}} P^{a c}\left(h_{0}^{e l}\right)
$$

The scattering operator is given by $s_{c}:=w_{+}\left(h^{e l}, h_{0}^{e l}\right)^{*} w_{-}\left(h^{e l}, h_{0}^{e l}\right)$. Let $\Pi\left(h_{0}^{e l, a c}\right)$ the spectral representation of $h_{0}^{e l, a c}$ introduced in Section2.6 If $\rho^{e l}$ and $q^{e l}$ are density and charge operators for $h_{0}^{e l}$, then the Landauer-Büttiker formula takes the form

$$
J_{\rho^{e l}, q^{e l}}^{s_{c}}=\frac{1}{2 \pi} \int_{\mathbb{R}} \operatorname{tr}\left(\rho_{a c}^{e l}(\lambda)\left(q_{a c}^{e l}-s_{c}(\lambda)^{*} q_{a c}^{e l}(\lambda) s_{c}(\lambda)\right)\right)
$$


where $\left\{s_{c}(\lambda)\right\}_{\lambda \in \mathbb{R}},\left\{q^{e l}(\lambda)\right\}_{\lambda \in \mathbb{R}}$ and $\left\{\rho^{e l}(\lambda)\right\}_{\lambda \in \mathbb{R}}$ are the scattering, charge and density matrices with respect to $\Pi\left(h_{0}^{e l, a c}\right)$, respectively. The condition that $\left(\left(h_{0}^{e l}\right)^{2}+\right.$ $\left.I_{\mathfrak{h}^{e l}}\right) \rho^{e l}$ is a bounded operator is superfluous because $h_{0}^{e l}$ is a bounded operator. For the same reason we have that every $p$-tempered charge $q^{e l}$ is in fact a charge, that means, $q^{e l}$ is a bounded self-adjoint operator.

The scattering system $s_{c}$ is a black-box model with reservoirs $\mathfrak{h}_{l}^{e l}$ and $\mathfrak{h}_{r}^{e l}$. Choosing

$$
\rho^{e l}=f_{l}\left(h^{e l}\right) \oplus f_{S}\left(h_{S}^{e l}\right) \oplus f_{r}\left(h_{r}^{e l}\right)
$$

where $f_{\alpha}(\cdot), \alpha=l, r$, are bounded Borel functions, and

$$
q^{e l}=g_{l}\left(h_{l}^{e l}\right) \oplus g_{S}\left(h_{S}^{e l}\right) \oplus g_{r}\left(h_{r}^{e l}\right),
$$

where $g_{\alpha}(\cdot), \alpha \in\{l, r\}$, are locally bounded Borel functions, then from (3.13) it follows that

$$
J_{\rho^{e l}, q^{e l}}^{s_{c}}=\frac{1}{2 \pi} \sum_{\substack{\alpha, \varkappa \in\{l, r\} \\ \alpha \neq \varkappa}} \int_{\mathbb{R}}\left(f_{\alpha}(\lambda)-f_{\varkappa}(\lambda)\right) g_{\alpha}(\lambda) \sigma_{c}(\lambda) d \lambda
$$

where $\left\{\sigma_{c}(\lambda)\right\}_{\lambda \in \mathbb{R}}$ is the channel cross-section from left to right and vice versa. Indeed, let $\left\{t_{c}(\lambda)\right\}_{\lambda \in \mathbb{R}}$ the transition matrix which corresponds to the transition operator $t_{c}:=s_{c}-I_{\mathfrak{h} e l}$. Obviously, one has $t_{c}(\lambda)=I_{\mathfrak{h}(\lambda)}-s_{c}(\lambda), \lambda \in \mathbb{R}$. Let $\left\{p_{\alpha}^{e l}(\lambda)\right\}_{\lambda \in \mathbb{R}}$ be the matrix which corresponds to the orthogonal projection $p_{\alpha}^{e l}$ from $\mathfrak{h}^{\text {el }}$ onto $\mathfrak{h}_{\alpha}^{\text {el }}$. Further, let $t_{r l}^{c}(\lambda):=p_{r}^{e l}(\lambda) t_{c}(\lambda) p_{l}^{e l}$ and $t_{l r}^{c}:=p_{l}^{e l}(\lambda) t_{c}(\lambda) p_{r}^{e l}$. Notice that both quantities are in fact scalar functions. Obviously, the channel cross-sections $\sigma_{l r}^{c}(\lambda)$ and $\sigma_{r l}^{c}(\lambda)$ at energy $\lambda \in \mathbb{R}$ are given by $\sigma_{c}(\lambda):=\sigma_{l r}^{c}(\lambda)=\left|t_{l r}^{c}(\lambda)\right|^{2}=\left|t_{r l}^{c}(\lambda)\right|^{2}=\sigma_{r l}^{c}(\lambda), \lambda \in \mathbb{R}$.

In particular, if $g_{l}(\lambda)=1$ and $g_{r}=0$, then

$$
J_{\rho^{e l}, q_{l}^{e l}}^{s_{c}}=\frac{1}{2 \pi} \int_{\mathbb{R}}\left(f_{l}(\lambda)-f_{r}(\lambda)\right) \sigma_{c}(\lambda) d \lambda,
$$

and $q_{l}^{e l}:=p_{l}^{e l}$. Following [1] $J_{\rho^{e},}^{s_{c}}, q_{l}^{e l}$ denotes the current entering the quantum dot from the left lead.

\subsection{Application to the $J C L$-model}

Let $S=\left\{H, H_{0}\right\}$ be now the $J C L$-model. Further, let $\rho$ and $Q$ be a density operator and a charge for $H_{0}$, respectively. Under these assumptions the current $J_{\rho, Q}^{S}$ is defined by

$$
J_{\rho, Q}^{\mathcal{S}}:=-i \operatorname{tr}\left(W_{-}\left(H, H_{0}\right)\left(I+H_{0}^{2}\right) \rho W_{-}\left(H, H_{0}\right)^{*} \frac{1}{H-i}[H, Q] \frac{1}{H+i}\right),
$$

and admits representation (3.4). If $Q$ is a $p$-tempered charge and $\left(H_{0}-i\right)^{p+2} \rho$ is a bounded operator, then the current $J_{\rho, Q}^{S}$ is defined in accordance with 3.77 and the Landauer-Büttiker formula (3.4) is valid, too. 
We introduce the intermediate scattering system $\mathcal{S}_{c}:=\left\{H, H_{c}\right\}$ where

$$
H_{c}:=h^{e l} \otimes I_{\mathfrak{h}^{p h}}+I_{\mathfrak{h}^{e l}} \otimes h^{p h}=H_{0}+V_{e l} .
$$

The Hamiltonian $H_{c}$ describes the coupling of the leads to quantum dot but under the assumption that the photon interaction is not switched on.

Obviously, $\mathcal{S}_{p h}:=\left\{H, H_{c}\right\}$ and $\mathcal{S}_{c}:=\left\{H_{c}, H_{0}\right\}$ are $\mathfrak{L}_{1}$-scattering systems. The corresponding scattering operators are denote by $S_{p h}$ and $S_{c}$, respectively. Let $\Pi\left(H_{c}^{a c}\right)=\left\{L^{2}\left(\mathbb{R}, d \lambda, \mathfrak{h}_{c}(\lambda)\right), \mathcal{M}, \Phi_{c}\right\}$ of $H_{c}^{a c}$ be a spectral representation of $H_{c}$. The scattering matrix of the scattering system $\left\{H, H_{c}\right\}$ with respect to $\Pi\left(H_{c}^{a c}\right)$ is denoted by $\left\{S_{p h}(\lambda)\right\}_{\lambda \in \mathbb{R}}$. The scattering matrix of the scattering system $\left\{H_{c}, H_{0}\right\}$ with respect to $\Pi\left(H_{0}^{a c}\right)=\left\{L^{2}\left(\mathbb{R}, d \lambda, \mathfrak{h}_{0}(\lambda)\right), \mathcal{M}, \Phi_{0}\right\}$ is denoted by $\left\{S_{c}(\lambda)\right\}_{\lambda \in \mathbb{R}}$.

Since $\mathcal{S}_{c}$ is a $\mathfrak{L}_{1}$-scattering system the wave operators $W_{ \pm}\left(H_{c}, H_{0}\right)$ exists and are complete and since $\Phi_{c} W_{ \pm}\left(H_{c}, H_{0}\right) \Phi_{0}^{*}$ commute with $\mathcal{M}$, there is a measurable families $\left\{W_{ \pm}(\lambda)\right\}_{\lambda \in \mathbb{R}}$ of isometries acting from $\mathfrak{h}_{0}(\lambda)$ onto $\mathfrak{h}_{c}(\lambda)$ for a.e. $\lambda \in \mathbb{R}$ such that

$$
\left(\Phi_{c} W_{ \pm}\left(H_{c}, H_{0}\right) \Phi_{0}^{*} \widehat{f}\right)(\lambda)=W_{ \pm}(\lambda) \widehat{f}(\lambda), \quad \lambda \in \mathbb{R}, \quad \widehat{f} \in L^{2}\left(\mathbb{R}, d \lambda, \mathfrak{h}_{0}(\lambda)\right) .
$$

The families $\left\{W_{ \pm}(\lambda)\right\}_{\lambda \in \mathbb{R}}$ are called wave matrices.

A straightforward computation shows that $\widehat{S}_{p h}:=W_{+}\left(H_{c}, H_{0}\right)^{*} S_{p h} W_{+}\left(H_{c}, H_{0}\right)$ commutes with $H_{0}$. Hence, with respect to the spectral representation $\Pi\left(H_{0}^{a c}\right)$ the operator $\widehat{S}_{p h}$ is unitarily equivalent to a multiplication induced by a measurable family $\left\{\widehat{S}_{p h}(\lambda)\right\}_{\lambda \in \mathbb{R}}$ of unitary operators in $\mathfrak{h}_{0}(\lambda)$. A straightforward computation shows that

$$
\widehat{S}_{p h}(\lambda)=W_{+}(\lambda)^{*} S_{p h}(\lambda) W_{+}(\lambda)
$$

for a.e. $\lambda \in \mathbb{R}$. Roughly speaking, $\left\{\widehat{S}_{p h}(\lambda)\right\}_{\lambda \in \mathbb{R}}$ is the scattering matrix of $S_{p h}$ with respect to the spectral representation $\Pi\left(H_{0}^{a c}\right)$.

Furthermore, let

$$
\rho^{c}:=W_{-}\left(H_{c}, H_{0}\right) \rho W_{-}\left(H_{c}, H_{0}\right)^{*}
$$

and

$$
Q^{c}:=W_{+}\left(H_{c}, H_{0}\right) Q W_{+}\left(H_{c}, H_{0}\right)^{*} .
$$

The operators $\rho^{c}$ and $Q^{c}$ are density and tempered charge operators for the scattering system $\mathcal{S}_{p h}$. Indeed, one easily verifies that $\rho^{c}$ and $Q^{c}$ are commute with $H_{c}$. Moreover, $\rho^{c}$ is non-negative. Furthermore, if $Q$ is a charge, then $Q^{c}$ is a charge, too. This gives rise to introduce the currents $J_{\rho, Q}^{c}:=J_{\rho, Q}^{S_{c}}$,

$$
J_{\rho, Q}^{c}:=-i \operatorname{tr}\left(W_{-}\left(H_{c}, H_{0}\right) \rho W_{-}\left(H_{c}, H_{0}\right)^{*} \frac{1}{H_{c}-i}\left[H_{c}, Q\right] \frac{1}{H_{c}+i}\right),
$$

and $J_{\rho, Q}^{p h}:=J_{\rho^{c}, Q^{c}}^{S_{p h}}$

$$
J_{\rho, Q}^{p h}:=-i \operatorname{tr}\left(W_{-}\left(H, H_{c}\right) \rho^{c} W_{-}\left(H, H_{c}\right)^{*} \frac{1}{H-i}\left[H, Q^{c}\right] \frac{1}{H+i}\right)
$$


which are well defined. If $Q$ is $p$-tempered charge and $\left(H_{0}-i\right)^{p+2} \rho$ is a bounded operator, then one easily checks that $Q^{c}$ is a $p$-tempered charge and $\left(H_{c}-i\right)^{p+2} \rho^{c}$ is a bounded operator. Hence the definition of the currents $J_{\rho^{c}, Q^{c}}^{S_{c}}$ can be extended to this case and the Landauer-Büttiker formula (3.4) holds.

Finally we note that the corresponding matrices $\left\{\rho_{a c}^{c}(\lambda)\right\}_{\lambda \in \mathbb{R}}$ and $\left\{Q_{a c}^{c}(\lambda)\right\}_{\lambda \in \mathbb{R}}$ are related to the matrices $\left\{\rho_{a c}(\lambda)\right\}_{\lambda \in \mathbb{R}}$ and $\left\{Q_{a c}(\lambda)\right\}_{\lambda \in \mathbb{R}}$ by

$$
\rho_{a c}^{c}(\lambda)=W_{-}(\lambda) \rho_{a c}(\lambda) W_{-}(\lambda)^{*} \quad \text { and } \quad Q_{a c}^{c}(\lambda)=W_{+}(\lambda) Q_{a c}(\lambda) W_{+}(\lambda)^{*}
$$

for a.e. $\lambda \in \mathbb{R}$.

Proposition 3.4 (Current decomposition) Let $\mathcal{S}=\left\{H, H_{0}\right\}$ be the JCL-model. Further, let $\rho$ and $Q$ be a density operator and a p-tempered charge, $p \in \mathbb{N}_{0}$, for $H_{0}$, respectively. If $\left(H_{0}-i\right)^{p+2} \rho$ is a bounded operator, then the decomposition

$$
J_{\rho, Q}^{\mathcal{S}}=J_{\rho, Q}^{c}+J_{\rho, Q}^{p h}
$$

holds where $J_{\rho, Q}^{c}$ and $J_{\rho, Q}^{p h}$ are given by (3.31) and (3.32).

In particular, let $\left\{S_{c}(\lambda)\right\}_{\lambda \in \mathbb{R}},\left\{\rho_{a c}(\lambda)\right\}_{\lambda \in \mathbb{R}}$ and $\left\{Q_{a c}(\lambda)\right\}_{\lambda \in \mathbb{R}}$ be scattering, density and charge matrices of $S_{c}, \rho$ and $Q$ with respect to $\Pi\left(H_{0}^{a c}\right)$ and let $\left\{S_{p h}(\lambda)\right\}_{\lambda \in \mathbb{R}}$, $\left\{\rho_{a c}^{c}(\lambda)\right\}_{\lambda \in \mathbb{R}}$ and $\left\{Q_{a c}^{c}(\lambda)\right\}_{\lambda \in \mathbb{R}}$ be the scattering, density and charge matrices of the scattering operator $S_{p h}$, density operator $\rho^{c}$, cf. (3.29), and charge operator $Q^{c}$, $c f$. (3.30), with respect to the spectral representation $\Pi\left(H_{c}^{a c}\right\}$. Then the representations

$$
\begin{aligned}
J_{\rho, Q}^{c} & :=\frac{1}{2 \pi} \int_{\mathbb{R}} \operatorname{tr}\left(\rho_{a c}(\lambda)\left(Q_{a c}(\lambda)-S_{c}(\lambda)^{*} Q_{a c}(\lambda) S_{c}(\lambda)\right) d \lambda,\right. \\
J_{\rho, Q}^{p h} & :=\frac{1}{2 \pi} \int_{\mathbb{R}} \operatorname{tr}\left(\rho_{a c}^{c}(\lambda)\left(Q_{a c}^{c}(\lambda)-S_{p h}(\lambda)^{*} Q_{a c}^{c}(\lambda) S_{p h}(\lambda)\right)\right) d \lambda,
\end{aligned}
$$

take place.

Proof. Since $\mathcal{S}_{c}$ and $\mathcal{S}_{p h}$ are $\mathfrak{L}_{1}$-scattering systems from Theorem 3.1 the representations (3.35) and (3.36) are easily follow. Taking into account 3.33) we get

$$
\begin{aligned}
& \operatorname{tr}\left(\rho_{a c}^{c}(\lambda)\left(Q_{a c}^{c}(\lambda)-S_{p h}(\lambda)^{*} Q_{a c}^{c}(\lambda) S_{p h}(\lambda)\right)\right)= \\
& \quad \operatorname{tr}\left(W_{-}(\lambda) \rho_{a c} W_{-}(\lambda)^{*}\left(W_{+}(\lambda) Q_{a c}(\lambda) W_{+}(\lambda)-S_{p h}(\lambda)^{*} Q_{a c}^{c}(\lambda) S_{p h}(\lambda)\right)\right) .
\end{aligned}
$$

Using $S_{c}(\lambda)=W_{+}(\lambda)^{*} W_{-}(\lambda)$ we find

$$
\begin{aligned}
& \operatorname{tr}\left(\rho_{a c}^{c}(\lambda)\left(Q_{a c}^{c}(\lambda)-S_{p h}(\lambda)^{*} Q_{a c}^{c}(\lambda) S_{p h}(\lambda)\right)\right)=\operatorname{tr}\left(\rho_{a c}(\lambda) \times\right. \\
& \left.\quad\left(S_{c}(\lambda)^{*} Q_{a c}(\lambda) S_{c}(\lambda)-W_{-}(\lambda)^{*} S_{p h}(\lambda)^{*} W_{+}(\lambda) Q_{a c}(\lambda) W_{+}(\lambda)^{*} S_{p h}(\lambda) W_{-}(\lambda)\right)\right) .
\end{aligned}
$$

Since $\left\{H_{c}, H_{0}\right\}$ and $\left\{H, H_{c}\right\}$ are $\mathfrak{L}_{1}$-scattering systems the existence of the wave operators $W_{ \pm}\left(H, H_{c}\right)$ and $W_{ \pm}\left(H_{c}, H_{0}\right)$ follows. Using the chain rule we find $W_{ \pm}\left(H, H_{0}\right)=W_{ \pm}\left(H, H_{c}\right) W_{ \pm}\left(H_{c}, H_{0}\right)$ which yields

$$
\begin{aligned}
S & =W_{+}\left(H, H_{0}\right)^{*} W_{+}\left(H, H_{0}\right) \\
& =W_{+}\left(H_{c}, H_{0}\right)^{*} W_{+}\left(H, H_{c}\right) W_{-}\left(H, H_{c}\right) W_{-}\left(H_{c}, H_{0}\right) \\
& =W_{+}\left(H_{c}, H_{0}\right)^{*} S_{p h} W_{-}\left(H_{c}, H_{0}\right) .
\end{aligned}
$$


Hence the scattering matrix $\{S(\lambda)\}_{\lambda \in \mathbb{R}}$ of $\left\{H, H_{0}\right\}$ admits the representation

$$
S(\lambda)=W_{+}(\lambda)^{*} S_{p h}(\lambda) W_{-}(\lambda), \quad \lambda \in \mathbb{R} .
$$

Inserting 3.38 into 3.37 we get

$$
J_{\rho, Q}^{p h}=\frac{1}{2 \pi} \int_{\mathbb{R}} \operatorname{tr}\left(\rho_{a c}(\lambda)\left(S_{c}(\lambda)^{*} Q_{a c}(\lambda) S_{c}(\lambda)-S(\lambda)^{*} Q_{a c}(\lambda) S(\lambda)\right)\right) d \lambda
$$

Using (3.39) we obtain

$$
J_{\rho, Q}^{c}+J_{\rho, Q}^{p h}=\frac{1}{2 \pi} \int_{\mathbb{R}} \operatorname{tr}\left(\rho_{a c}(\lambda)\left(Q_{a c}(\lambda)-S(\lambda)^{*} Q_{a c}(\lambda) S(\lambda)\right)\right) d \lambda .
$$

Finally, taking into account (3.4) we obtain (3.34).

\section{Remark 3.5}

(i) The current $J_{\rho, Q}^{c}$ is due to the coupling of the leads to the quantum dot and is therefore called the contact induced current.

(ii) The current $J_{\rho, Q}^{p h}$ is due to the interaction of photons with electrons and is therefore called the photon induced current. Notice the this current is calculated under the assumption that the leads already contacted to the dot.

Corollary 3.6 Let the assumptions of Proposition 3.4 be satisfied. With respect to the spectral representation $\Pi\left(H_{0}^{a c}\right)$ of $H_{0}^{a c}$ the photon induced current $J_{\rho, Q}^{p h}$ can be represented by

$$
J_{\rho, Q}^{p h}:=\frac{1}{2 \pi} \int_{\mathbb{R}} \operatorname{tr}\left(\widehat{\rho}_{a c}(\lambda)\left(Q_{a c}(\lambda)-\widehat{S}_{p h}(\lambda)^{*} Q_{a c}(\lambda) \widehat{S}_{p h}(\lambda)\right)\right) d \lambda
$$

where the measurable families $\left\{\widehat{S}_{p h}(\lambda)\right\}_{\lambda \in \mathbb{R}}$ and $\left\{\widehat{\rho}_{a c}(\lambda)\right\}_{\lambda \in \mathbb{R}}$ are given by (3.28) and

$$
\widehat{\rho}_{a c}(\lambda):=S_{c}(\lambda) \rho_{a c}(\lambda) S_{c}(\lambda)^{*} \quad \lambda \in \mathbb{R},
$$

respectively.

Proof. Using (3.33) and $S_{c}(\lambda)=W_{+}(\lambda)^{*} W_{-}(\lambda)$ we find

$$
\begin{array}{r}
\operatorname{tr}\left(\rho_{a c}^{c}(\lambda)\left(Q_{a c}^{c}(\lambda)-S_{p h}(\lambda)^{*} Q_{a c}^{c}(\lambda) S_{p h}(\lambda)\right)\right)=\operatorname{tr}\left(S_{c}(\lambda) \rho_{a c}(\lambda) S_{c}(\lambda)^{*} \times\right. \\
\left.\left(Q_{a c}(\lambda)-W_{+}(\lambda)^{*} S_{p h}(\lambda)^{*} W_{+}(\lambda) Q_{a c}(\lambda) W_{+}(\lambda)^{*} S_{p h}(\lambda) W_{+}(\lambda)\right)\right) .
\end{array}
$$

Taking into account the representations (3.28) and (3.41) we get

$$
\begin{aligned}
& \operatorname{tr}\left(\rho_{a c}^{c}(\lambda)\left(Q_{a c}^{c}(\lambda)-S_{p h}(\lambda)^{*} Q_{a c}^{c}(\lambda) S_{p h}(\lambda)\right)\right)= \\
& \quad \operatorname{tr}\left(S_{c}(\lambda) \rho_{a c}(\lambda) S_{c}(\lambda)^{*}\left(Q_{a c}(\lambda)-\widehat{S}_{p h}(\lambda)^{*} Q_{a c}(\lambda) \widehat{S}_{p h}(\lambda)\right)\right)
\end{aligned}
$$

which immediately yields 3.40 .

Remark 3.7 In the following we call $\left\{\widehat{\rho}_{a c}(\lambda)\right\}_{\lambda \in \mathbb{R}}$, cf. 3.41, the photon modified electron density matrix. Notice that $\left\{\widehat{\rho}_{a c}(\lambda)\right\}_{\lambda \in \mathbb{R}}$ might be non-diagonal even if the electron density matrix $\left\{\rho_{a c}(\lambda)\right\}_{\lambda \in \mathbb{R}}$ is diagonal. 


\section{Analysis of currents}

In the following we analyze currents $J_{\rho, Q}^{c}$ and $J_{\rho, Q}^{p h}$ under the assumption that $\rho$ and $Q$ have the tensor product structure

$$
\rho=\rho^{e l} \otimes \rho^{p h} \quad \text { and } \quad Q=q^{e l} \otimes q^{p h}
$$

where $\rho^{e l}$ and $\rho^{p h}$ as well as $q^{e l}$ and $q^{p h}$ are density operators and (tempered) charges for $h_{0}^{e l}$ and $h^{p h}$, respectively. Since $\rho^{p h}$ commutes with $h^{p h}$, which is discrete, the operator $\rho^{p h}$ has the form

$$
\rho^{p h}=\rho^{p h}(n)\left(\cdot, \Upsilon_{n}\right) \Upsilon_{n}, \quad n \in \mathbb{N}_{0},
$$

where $\rho^{p h}(n)$ are non-negative numbers. Similarly, $q^{p h}$ can be represented by

$$
q^{p h}=q^{p h}(n)\left(\cdot, \Upsilon_{n}\right) \Upsilon_{n}, \quad n \in \mathbb{N}_{0},
$$

where $q^{p h}(n)$ are real numbers.

Lemma 4.1 Let $\mathcal{S}=\left\{H, H_{0}\right\}$ be the JCL-model. Assume that $\rho \neq 0$ and $Q$ have the structure (4.1) where $\rho^{\text {el }}$ is a density operator and $q^{e l}$ is a charge for $h_{0}^{e l}$.

(i) The operator $\left(H_{0}-i\right)^{p+2} \rho, p \in \mathbb{N}_{0}$, is bounded if and only if the condition

$$
\sup _{n \in \mathbb{N}_{0}} \rho^{p h}(n) n^{p+2}<\infty
$$

is satisfied.

(ii) The charge $Q$ is p-tempered if and only if

$$
\sup _{n \in \mathbb{N}}\left|q^{p h}(n)\right| n^{-p}<\infty .
$$

is valid

Proof. (i) The operator $\left(H_{0}-i\right)^{p+2} \rho$ admits the representation

$$
\left(H_{0}-i\right)^{p+2} \rho=\bigoplus_{p \in \mathbb{N}_{0}} \rho^{p h}(n)\left(h_{0}^{e l}+n \omega-i\right)^{p+2} \rho^{e l} .
$$

We have

$$
\begin{aligned}
\left\|\left(H_{0}-i\right)^{p+2} \rho\right\|_{\mathfrak{L}(\mathfrak{H})} & =\sup _{p \in \mathbb{N}_{0}} \rho^{p h}(n)\left\|\left(h_{0}^{e l}+n \omega-i\right)^{p+2} \rho^{e l}\right\|_{\mathfrak{L}\left(\mathfrak{h}^{e l}\right)} \\
& =\sup _{p \in \mathbb{N}_{0}} \rho^{p h}(n) n^{p+2} n^{-(p+2)}\left\|\left(h_{0}^{e l}+n \omega-i\right)^{p+2} \rho^{e l}\right\|_{\mathfrak{L}\left(\mathfrak{h}^{e l}\right)} .
\end{aligned}
$$

Since $\lim _{n \rightarrow \infty} n^{-(p+2)}\left\|\left(h_{0}^{e l}+n \omega-i\right)^{p+2} \rho^{e l}\right\|_{\mathfrak{L}\left(\mathfrak{h}^{e l}\right)}=\omega^{p+2}\left\|\rho^{e l}\right\|_{\mathfrak{L}\left(\mathfrak{h}^{e l}\right)}$ we get for sufficiently large $n \in \mathbb{N}_{0}$ that

$$
\frac{\omega^{p+2}}{2}\left\|\rho^{e l}\right\|_{\mathfrak{L}\left(\mathfrak{h}^{e l}\right)} \leq n^{-(p+2)}\left\|\left(h_{0}^{e l}+n \omega-i\right)^{p+2} \rho^{e l}\right\|_{\mathfrak{L}\left(\mathfrak{h}^{e l}\right)} .
$$


Using that and (4.6) we immediately obtain (4.4). Conversely, from (4.6) and 4.4) we obtain that $\left(H_{0}-i\right)^{p+2} \rho$ is a bounded operator.

(ii) As above we have

$$
Q\left(H_{0}-i\right)^{-p}=\bigoplus_{n \in \mathbb{N}_{0}} q^{p h}(n) q^{e l}
$$

Hence

$$
\left\|Q\left(H_{0}-i\right)^{-p}\right\|_{\mathfrak{L}(\mathfrak{H})}=\sup _{n \in \mathbb{N}_{0}} \mid q^{p h}(n)\left\|q^{e l}\left(h_{0}^{e l}+n \omega-i\right)^{-p}\right\|_{\mathfrak{L}\left(\mathfrak{h}^{e l}\right)} .
$$

Since $\lim _{n \rightarrow \infty} n^{p}\left\|\left(h_{0}^{e l}+n \omega-i\right)^{-p}\right\|_{\mathfrak{L}\left(\mathfrak{h}^{e l}\right)}=\omega^{-p}\left\|q^{e l}\right\|_{\mathfrak{L}\left(\mathfrak{h}^{e l}\right)}$ we get similarly as above that (4.5) holds. The converse is obvious.

\subsection{Contact induced current}

Let us recall that $\mathcal{S}_{c}=\left\{H_{c}, H_{0}\right\}$ is a $\mathfrak{L}_{1}$-scattering system. An obvious computations shows that

$$
W_{ \pm}\left(H_{c}, H_{0}\right)=w_{ \pm}\left(h^{e l}, h_{0}^{e l}\right) \otimes I_{\mathfrak{h}^{p h}}
$$

where $w_{ \pm}\left(h^{e l}, h_{0}^{e l}\right)$ is given by (3.24). Hence

$$
S_{c}=s_{c} \otimes I_{\mathfrak{h}^{p h}}, \quad \text { where } \quad s_{c}:=w_{+}\left(h_{c}^{e l}, h_{0}^{e l}\right)^{*} w_{-}\left(h_{c}^{e l}, h_{0}^{e l}\right) .
$$

Proposition 4.2 Let $\mathcal{S}=\left\{H, H_{0}\right\}$ be the JCL-model. Assume that $\rho$ and $Q$ are given by (4.1) where $\rho^{e l}$ and $q^{\text {el }}$ are density and charge operators for $h_{0}^{e l}$ and $\rho^{p h}$ and $q^{p h}$ for $h^{p h}$, respectively. If for some $p \in \mathbb{N}_{0}$ the conditions (4.4) and (4.5) are satisfied, then the current $J_{\rho, Q}^{c}$ is well defined and admits the representation

$$
J_{\rho, Q}^{c}=\gamma J_{\rho^{e l}, q^{e l}}^{s_{c}}, \quad \gamma:=\sum_{n \in \mathbb{N}_{0}} q^{p h}(n) \rho^{p h}(n)
$$

where $J_{\rho^{e l}, q^{e l}}^{s_{c}}$ is defined by 3.2). In particular, if $\operatorname{tr}\left(\rho^{p h}\right)=1$ and $q^{p h}=I_{\mathfrak{h}^{p h}}$, then $J_{\rho, Q}^{c}=J_{\rho^{e l}, q^{e l}}^{s_{c}}$.

Proof. First of all we note that by lemma 4.1 the operator $\left(H_{0}-i\right)^{p+2} \rho$ is bounded and $Q$ is $p$-tempered. Hence the current $J_{\rho, Q}^{S_{c}}$ is correctly defined and the Landauer-Büttiker formula (3.4) is valid.

With respect to the spectral representation $\Pi\left(H_{0}^{a c}\right)$ of Lemma2.12 the charge matrix $\left\{Q_{a c}(\lambda)\right\}_{\lambda \in \mathbb{R}}$ of $Q_{a c}=q_{a c}^{e l} \otimes q^{p h}$ admits the representation

$$
Q_{a c}(\lambda)=\bigoplus_{n \in \mathbb{N}_{0}} q_{a c}^{e l}(\lambda-n \omega) q^{p h}(n), \quad \lambda \in \mathbb{R} .
$$


Since $S_{c}=s_{c} \otimes I_{\mathfrak{h}^{p h}}$ the scattering matrix $\left\{S_{c}(\lambda)\right\}_{\lambda \in \mathbb{R}}$ admits the representation

$$
S_{c}(\lambda)=\bigoplus_{n \in \mathbb{N}_{0}} s_{c}(\lambda-n \omega), \quad \lambda \in \mathbb{R}
$$

Hence

$$
\begin{aligned}
& Q_{a c}(\lambda)-S_{c}(\lambda)^{*} Q_{a c}(\lambda) S_{c}(\lambda)= \\
& \bigoplus_{n \in \mathbb{N}_{0}} q^{p h}(n)\left(q_{a c}^{e l}(\lambda-n \omega)-s_{c}(\lambda-n \omega)^{*} q_{a c}^{e l}(\lambda-\omega n) s_{c}(\lambda-n \omega)\right) .
\end{aligned}
$$

Moreover, the density matrix $\left\{\rho_{a c}(\lambda)\right\}_{\lambda \in \mathbb{R}}$ admits the representation

$$
\rho_{a c}(\lambda)=\bigoplus_{n \in \mathbb{N}_{0}} \rho^{p h}(n) \rho_{a c}^{e l}(\lambda-n \omega)
$$

Inserting 4.10) into 4.9] we find

$$
\begin{aligned}
& \rho^{a c}(\lambda)\left(Q_{a c}(\lambda)-S_{c}(\lambda)^{*} Q_{a c}(\lambda) S_{c}(\lambda)\right)=\bigoplus_{n \in \mathbb{N}_{0}} q^{p h}(n) \rho^{p h}(n) \times \\
& \quad \rho_{a c}^{e l}(\lambda-n \omega)\left(q_{a c}^{e l}(\lambda-\omega n)-s_{c}(\lambda-n \omega)^{*} q_{a c}^{e l}(\lambda-\omega n) s_{c}(\lambda-n \omega)\right)
\end{aligned}
$$

Since $\gamma=\sum_{n \in \mathbb{N}_{0}} q^{p h}(n) \rho^{p h}(n)$ is absolutely convergent by (4.4) and (4.5) we obtain that

$$
\begin{aligned}
& \operatorname{tr}\left(\rho^{a c}(\lambda)\left(Q_{a c}(\lambda)-S_{c}(\lambda)^{*} Q_{a c}(\lambda) S_{c}(\lambda)\right)\right)=\sum_{n \in \mathbb{N}_{0}} q^{p h}(n) \rho^{p h}(n) \times \\
& \operatorname{tr}\left(\rho_{a c}^{e l}(\lambda-n \omega)\left(q_{a c}^{e l}(\lambda-\omega n)-s_{c}(\lambda-n \omega)^{*} q_{a c}^{e l}(\lambda-\omega n) s_{c}(\lambda-n \omega)\right)\right)
\end{aligned}
$$

Obviously, we have

$$
\begin{gathered}
\left|\operatorname{tr}\left(\rho_{a c}^{e l}(\lambda-n \omega)\left(q_{a c}^{e l}(\lambda-\omega n)-s_{c}(\lambda-n \omega)^{*} q_{a c}^{e l}(\lambda-\omega n) s_{c}(\lambda-n \omega)\right)\right)\right| \leq \\
4\left\|\rho_{a c}^{e l}(\lambda-n \omega)\right\|_{\mathfrak{L}\left(\mathfrak{h}_{n}(\lambda)\right)}\left\|q_{a c}^{e l}(\lambda-n \omega)\right\|_{\mathfrak{L}\left(\mathfrak{h}_{n}(\lambda)\right)}, \quad \lambda \in \mathbb{R} .
\end{gathered}
$$

We insert (4.11) into the Landauer-Büttiker formula (3.35). Using (4.4) and (4.5) as well as

$$
\int_{\mathbb{R}}\left\|\rho_{a c}^{e l}(\lambda)\right\|_{\mathfrak{L}\left(\mathfrak{h}_{n}(\lambda)\right)}\left\|q_{a c}^{e l}(\lambda)\right\|_{\mathfrak{L}\left(\mathfrak{h}_{n}(\lambda)\right)} d \lambda<\infty
$$

we see that we can interchange the integral and the sum. Doing so we get

$$
\begin{aligned}
J_{\rho, Q}^{c} & =\sum_{n \in \mathbb{N}_{0}} q^{p h}(n) \rho^{p h}(n) \frac{1}{2 \pi} \int_{\mathbb{R}} \operatorname{tr}\left(\rho_{a c}^{e l}(\lambda-n \omega) \times\right. \\
& \left.\left(q_{a c}^{e l}(\lambda-\omega n)-s_{c}(\lambda-n \omega)^{*} q_{a c}^{e l}(\lambda-\omega n) s_{c}(\lambda-n \omega)\right)\right) d \lambda .
\end{aligned}
$$

Using (3.25) we prove 4.7).

If $\operatorname{tr}\left(\rho^{p h}\right)=1$, then $\sum_{\mathbb{N}_{0}} \rho^{p h}(n)=1$. Further, if $\rho^{p h}=I_{\mathfrak{h}^{p h}}$, then $q^{p h}(n)=1$. Hence $\gamma=1$. 


\subsection{Photon induced current}

To calculate the current $J_{\rho, Q}^{p h}$ we used the representation 3.40). We set

$$
\widehat{S}_{m n}^{p h}(\lambda):=P_{m}(\lambda) \widehat{S}_{p h}(\lambda)\left\lceil\mathfrak{h}_{n}(\lambda), \quad \lambda \in \mathbb{R} .\right.
$$

where $\left\{\widehat{S}_{p h}(\lambda)\right\}_{\lambda \in \mathbb{R}}$ is defined by $(3.28)$ and $P_{m}(\lambda)$ is the orthogonal projection from $\mathfrak{h}(\lambda)$, cf. [2.41), onto $\mathfrak{h}_{m}(\lambda):=\mathfrak{h}^{\text {el }}(\lambda-m \omega), \lambda \in \mathbb{R}$.

Proposition 4.3 Let $\mathcal{S}=\left\{H, H_{0}\right\}$ be the JCL-model. Assume that $\rho$ and $Q$ are given by (4.1) where $\rho^{e l}$ and $q^{e l}$ are density and charge operators for $h_{0}^{e l}$ and $\rho^{p h}$ and $q^{p h}$ for $h^{p h}$, respectively. If for some $p \in \mathbb{N}_{0}$ the conditions (4.4) and (4.5) are satisfied, then the current $J_{\rho, Q}^{p h}$ is well-defined and admits the representation

$$
\begin{aligned}
J_{\rho, Q}^{p h}= & \sum_{m \in \mathbb{N}_{0}} \rho^{p h}(m) \sum_{n \in \mathbb{N}_{0}} q^{p h}(n) \frac{1}{2 \pi} \int_{\mathbb{R}} d \lambda \operatorname{tr}\left(\widehat{\rho}_{a c}^{e l}(\lambda-m \omega) \times\right. \\
& \left.\left(q_{a c}^{e l}(\lambda-n \omega) \delta_{m n}-\widehat{S}_{n m}^{p h}(\lambda)^{*} q_{a c}^{e l}(\lambda-n \omega) \widehat{S}_{n m}^{p h}(\lambda)\right)\right) .
\end{aligned}
$$

where $\left\{\hat{\rho}_{a c}^{e l}(\lambda)\right\}_{\lambda \in \mathbb{R}}$ is the photon modified electron density defined, cf. [3.41), which takes the form

$$
\hat{\rho}_{a c}^{e l}(\lambda)=s_{c}(\lambda) \rho^{e l}(\lambda) s_{c}(\lambda)^{*}, \quad \lambda \in \mathbb{R} .
$$

Proof. By Lemma 4.1 we get that that the charge $Q$ is $p$-tempered and $\left(H_{0}-i\right)^{p} \rho$ is a bounded operator. By Corollary 3.2 the current $J_{\rho, Q}^{p h}:=J_{\rho^{c}, Q^{c}}^{S_{p h}}$ is well-defined.

Since $\left(Q_{a c}(\lambda)-\widehat{S}_{p h}(\lambda)^{*} Q_{a c}(\lambda) \widehat{S}_{p h}(\lambda)\right)$ is a trace class operator for $\lambda \in \mathbb{R}$ we get from (3.40) and (4.10) that

$$
\begin{aligned}
& \operatorname{tr}\left(\widehat{\rho}_{a c}(\lambda)\left(Q_{a c}(\lambda)-\widehat{S}_{p h}(\lambda)^{*} Q_{a c}(\lambda) \widehat{S}_{p h}(\lambda)\right)\right)=\sum_{m \in \mathbb{N}_{0}} \rho^{p h}(m) \times \\
& \quad \operatorname{tr}\left(\widehat{\rho}^{e l}(\lambda-m \omega) P_{m}(\lambda)\left(Q_{a c}(\lambda)-\widehat{S}_{p h}(\lambda)^{*} Q_{a c}(\lambda) \widehat{S}_{p h}(\lambda)\right) P_{m}(\lambda)\right)
\end{aligned}
$$

Further we have

$$
\begin{aligned}
P_{m}(\lambda) & \left(Q_{a c}(\lambda)-\widehat{S}_{p h}(\lambda)^{*} Q_{a c}(\lambda) \widehat{S}_{p h}(\lambda)\right) P_{m}(\lambda) \\
& =q^{p h}(m)\left(q^{e l}(\lambda-m \omega)-P_{m}(\lambda) \widehat{S}_{p h}(\lambda)^{*} Q_{a c}(\lambda) \widehat{S}_{p h}(\lambda)\right) P_{m}(\lambda) \\
& =q^{p h}(m) q^{e l}(\lambda-m \omega)-\sum_{n \in \mathbb{N}_{0}} q^{p h}(n) \widehat{S}_{n m}^{p h}(\lambda)^{*} q^{e l}(\lambda-n \omega) \widehat{S}_{n m}^{p h}(\lambda)
\end{aligned}
$$

for $\lambda \in \mathbb{R}$ where $\widehat{S}_{n m}^{p h}(\lambda)^{*}:=P_{n}(\lambda) \widehat{S}_{p h}(\lambda) P_{m}(\lambda), \lambda \in \mathbb{R}$. Notice that $\sum_{n \in \mathbb{N}_{0}}$ is a sum with a finite number of summands. Hence

$$
\begin{aligned}
& \operatorname{tr}\left(\widehat{\rho}_{a c}(\lambda)\left(Q_{a c}(\lambda)-\widehat{S}_{p h}(\lambda)^{*} Q_{a c}(\lambda) \widehat{S}_{p h}(\lambda)\right)\right)=\sum_{m \in \mathbb{N}_{0}} \rho^{p h}(m) \sum_{n \in \mathbb{N}_{0}} q^{p h}(n) \times \\
& \operatorname{tr}\left(\widehat{\rho}^{e l}(\lambda-m \omega)\left(q^{e l}(\lambda-m \omega) \delta_{m n}-\widehat{S}_{n m}^{p h}(\lambda)^{*} q^{e l}(\lambda-n \omega) \widehat{S}_{n m}^{p h}(\lambda)\right)\right)
\end{aligned}
$$


We are going to show that

$$
\begin{aligned}
\sum_{m \in \mathbb{N}_{0}} \rho^{p h}(m) & \sum_{n \in \mathbb{N}_{0}}\left|q^{p h}(n)\right| \int_{\mathbb{R}} \mid \operatorname{tr}\left(\widehat{\rho}^{e l}(\lambda-m \omega) \times\right. \\
& \left.\left(q^{e l}(\lambda-m \omega) \delta_{m n}-\widehat{S}_{n m}^{p h}(\lambda)^{*} q^{e l}(\lambda-n \omega) \widehat{S}_{n m}^{p h}(\lambda)\right)\right) \mid d \lambda<\infty .
\end{aligned}
$$

Obviously one has the estimate

$$
\begin{aligned}
& \left|\operatorname{tr}\left(\widehat{\rho}^{e l}(\lambda-m \omega)\left(q^{e l}(\lambda-m \omega) \delta_{m n}-\widehat{S}_{n m}^{p h}(\lambda)^{*} q^{e l}(\lambda-n \omega) \widehat{S}_{n m}^{p h}(\lambda)\right)\right)\right| \leq \\
& 2\left\|\widehat{\rho}^{e l}(\lambda-m \omega)\right\|_{\mathfrak{L}\left(\mathfrak{h}_{m}(\lambda)\right)}\left(\left\|q^{e l}(\lambda-m \omega)\right\|_{\mathfrak{L}\left(\mathfrak{h}_{m}(\lambda)\right)} \delta_{n m}+\left\|q^{e l}(\lambda-n \omega)\right\|_{\mathfrak{L}\left(\mathfrak{h}_{n}(\lambda)\right)}\right) .
\end{aligned}
$$

Further, we get

$$
\begin{gathered}
\int_{\lambda \in \mathbb{R}}\left\|\hat{\rho}^{e l}(\lambda-m \omega)\right\|_{\mathfrak{L}\left(\mathfrak{h}_{m}(\lambda)\right)}\left\|q^{e l}(\lambda-m \omega)\right\|_{\mathfrak{L}\left(\mathfrak{h}_{m}(\lambda)\right)} \delta_{n m} \leq \\
\int_{\lambda \in \mathbb{R}}\left\|\hat{\rho}^{e l}(\lambda)\right\|_{\mathfrak{L}\left(\mathfrak{h}_{m}(\lambda)\right)}\left\|q^{e l}(\lambda)\right\|_{\mathfrak{L}\left(\mathfrak{h}_{m}(\lambda)\right)} d \lambda
\end{gathered}
$$

and

$$
\begin{gathered}
\int_{\mathbb{R}}\left\|\hat{\rho}^{e l}(\lambda-m \omega)\right\|_{\mathfrak{L}\left(\mathfrak{h}_{m}(\lambda)\right)}\left\|q^{e l}(\lambda-n \omega)\right\|_{\mathfrak{L}\left(\mathfrak{h}_{n}(\lambda)\right)} d \lambda \leq \\
\left\|q_{a c}^{e l}\right\|_{\mathfrak{L}\left(\mathfrak{h}^{e l}\right)} \int_{\lambda \in \mathbb{R}}\left\|\hat{\rho}^{e l}(\lambda-(m-n) \omega)\right\|_{\mathfrak{L}\left(\mathfrak{h}_{m-n}(\lambda)\right)} d \lambda
\end{gathered}
$$

If the conditions (4.4) and 4.5) are satisfied, then

$$
\sum_{m \in \mathbb{N}_{0}} \rho^{p h}(m)\left|q^{p h}(m)\right| \int_{\mathbb{R}}\left\|\widehat{\rho}^{e l}(\lambda)\right\|_{\mathfrak{L}\left(\mathfrak{h}_{m}(\lambda)\right)}\left\|q^{e l}(\lambda)\right\|_{\mathfrak{L}\left(\mathfrak{h}_{m}(\lambda)\right)} d \lambda<\infty
$$

Further, we have

$$
\begin{aligned}
& \sum_{m \in \mathbb{N}_{0}} \rho^{p h}(m) \sum_{n \in \mathbb{N}_{0}}\left|q^{p h}(n)\right| \int_{\lambda \in \mathbb{R}}\left\|\hat{\rho}^{e l}(\lambda-(m-n) \omega)\right\|_{\mathfrak{L}\left(\mathfrak{h}_{m-n}(\lambda)\right)} d \lambda \leq \\
& \left(v_{\max }-v_{\min }+4\right)\left\|\rho_{a c}^{e l}\right\|_{\mathfrak{L}\left(\mathfrak{h}^{e l}\right)} \sum_{m \in \mathbb{N}_{0}} \rho^{p h}(m) \sum_{|m-n| \leq d_{\max }}\left|q^{p h}(n)\right|<\infty
\end{aligned}
$$

where $d_{\max }$ is introduced by Lemma2.12 To prove

$$
\sum_{m \in \mathbb{N}_{0}} \rho^{p h}(m) \sum_{|m-n| \leq d_{\max }}\left|q^{p h}(n)\right|<\infty
$$

we use again (4.4) and (4.5). The last step admits to interchange the integral and the sums which immediately proves 4.12 
Corollary 4.4 Let $\mathcal{S}=\left\{H, H_{0}\right\}$ be the JCL-model. Assume that $\rho$ and $Q$ are given by (4.1) where $\rho^{e l}$ and $q^{e l}$ are density and charge operators for $h_{0}^{e l}$ and $\rho^{p h}$ and $q^{p h}$ for $h^{p h}$, respectively. If $\rho^{e l}$ is an equilibrium state, i.e. $\rho^{e l}=f^{e l}\left(h_{0}^{e l}\right)$, then

$$
\begin{aligned}
J_{\rho, Q}^{p h}= & \sum_{m, n \in \mathbb{N}_{0}} q^{p h}(n) \frac{1}{2 \pi} \int_{\mathbb{R}}\left(\rho^{p h}(n) f^{e l}(\lambda-n \omega)-\rho^{p h}(m) f^{e l}(\lambda-m \omega)\right) \times \\
& \operatorname{tr}\left(\widehat{S}_{n m}^{p h}(\lambda)^{*} q_{a c}^{e l}(\lambda-n \omega) \widehat{S}_{n m}^{p h}(\lambda)\right) d \lambda .
\end{aligned}
$$

Proof. From (4.12) we get

$$
\begin{aligned}
J_{\rho, Q}^{p h}= & \sum_{n \in \mathbb{N}_{0}} q^{p h}(n) \sum_{m \in \mathbb{N}_{0}} \rho^{p h}(m) \frac{1}{2 \pi} \int_{\mathbb{R}} d \lambda f^{e l}(\lambda-m \omega) \times \\
& \operatorname{tr}\left(q_{a c}^{e l}(\lambda-n \omega) \delta_{m n}-\widehat{S}_{n m}^{p h}(\lambda)^{*} q_{a c}^{e l}(\lambda-n \omega) \widehat{S}_{n m}^{p h}(\lambda)\right) .
\end{aligned}
$$

Hence

$$
\begin{aligned}
& J_{\rho, Q}^{p h}=\sum_{n \in \mathbb{N}_{0}} q^{p h}(n) \frac{1}{2 \pi} \int_{\mathbb{R}} d \lambda \sum_{m \in \mathbb{N}_{0}} \rho^{p h}(m) f^{e l}(\lambda-m \omega) \times \\
& \operatorname{tr}\left(q_{a c}^{e l}(\lambda-n \omega) \delta_{m n}-\widehat{S}_{n m}^{p h}(\lambda)^{*} q_{a c}^{e l}(\lambda-n \omega) \widehat{S}_{n m}^{p h}(\lambda)\right) .
\end{aligned}
$$

This gives

$$
\begin{aligned}
J_{\rho, Q}^{p h}= & \sum_{n \in \mathbb{N}_{0}} q^{p h}(n) \frac{1}{2 \pi} \int_{\mathbb{R}} d \lambda\left(\rho^{p h}(n) f^{e l}(\lambda-n \omega) \operatorname{tr}\left(q_{a c}^{e l}(\lambda-n \omega)\right)-\right. \\
& \left.\sum_{m \in \mathbb{N}_{0}} \rho^{p h}(m) f^{e l}(\lambda-m \omega) \operatorname{tr}\left(\widehat{S}_{n m}^{p h}(\lambda)^{*} q_{a c}^{e l}(\lambda-n \omega) \widehat{S}_{n m}^{p h}(\lambda)\right)\right) .
\end{aligned}
$$

Since

$$
\begin{gathered}
\sum_{m \in \mathbb{N}_{0}} \rho^{p h}(m) f^{e l}(\lambda-m \omega) \operatorname{tr}\left(\widehat{S}_{n m}^{p h}(\lambda)^{*} q_{a c}^{e l}(\lambda-n \omega) \widehat{S}_{n m}^{p h}(\lambda)\right)= \\
\sum_{m \in \mathbb{N}_{0}}\left(\rho^{p h}(m) f^{e l}(\lambda-m \omega)-\rho^{p h}(n) f^{e l}(\lambda-n \omega)\right) \times \\
\operatorname{tr}\left(\widehat{S}_{n m}^{p h}(\lambda)^{*} q_{a c}^{e l}(\lambda-n \omega) \widehat{S}_{n m}^{p h}(\lambda)\right)+ \\
\rho^{p h}(n) f^{e l}(\lambda-n \omega) \sum_{m \in \mathbb{N}_{0}} \operatorname{tr}\left(\widehat{S}_{n m}^{p h}(\lambda)^{*} q_{a c}^{e l}(\lambda-n \omega) \widehat{S}_{n m}^{p h}(\lambda)\right)
\end{gathered}
$$

Inserting this into (4.15) we obtain 4.14). 


\section{Electron and photon currents}

\subsection{Electron current}

To calculate the electron current induced by contacts and photons contact we make the following choice throughout this section. We set

$$
Q_{\alpha}^{e l}:=q_{\alpha}^{e l} \otimes q^{p h}, \quad q_{\alpha}^{e l}:=-\mathfrak{e} p_{\alpha}^{e l} \quad \text { and } \quad q^{p h}:=I_{\mathfrak{h}^{p h}}, \quad \alpha \in\{l, r\},
$$

where $p_{\alpha}^{e l}$ denotes the orthogonal projection from $\mathfrak{h}^{e l}$ onto $\mathfrak{h}_{\alpha}^{e l}$. By $\mathfrak{e}>0$ we denote the magnitude of the elementary charge. Since $p_{\alpha}^{e l}$ commutes with $h_{\alpha}^{e l}$ one easily verifies that $Q_{\alpha}^{e l}$ commutes with $H_{0}$ which shows that $Q_{\alpha}^{e l}$ is a charge. Following [1] the flux related to $Q_{\alpha}^{e l}$ gives us the electron current $J_{\rho, Q_{\alpha}^{e l}}^{\mathcal{S}}$ entering the lead $\alpha$ from the sample. Notice $Q_{\alpha}^{e l}=-\mathfrak{e} P_{\alpha}$ where $P_{\alpha}$ is the orthogonal projection from $\mathfrak{H}$ onto $\mathfrak{H}_{\alpha}:=\mathfrak{h}_{\alpha}^{e l} \otimes$ $\mathfrak{h}^{p h}$. Since $q^{p h}=I_{\mathfrak{h}^{p h}}$ the condition 4.5) is immediately satisfied for any $p \geq 0$.

Let $f(\cdot): \mathbb{R} \longrightarrow \mathbb{R}$ be a non-negative bounded measurable function. We set

$$
\rho^{e l}=\rho_{l}^{e l} \oplus \rho_{S}^{e l} \oplus \rho_{r}^{e l}, \quad \rho_{\alpha}^{e l}:=f\left(h_{\alpha}^{e l}-\mu_{\alpha}\right), \quad \alpha \in\{l, r\} .
$$

and $\rho=\rho^{e l} \otimes \rho^{p h}$. By $\mu_{\alpha}$ the chemical potential of the lead $\alpha$ is denoted. In applications one sets $f(\lambda):=f_{F D}(\lambda), \lambda \in \mathbb{R}$, where $f_{F D}(\lambda)$ is the so-called Fermi-Dirac distribution given by (3.21). If $\beta=\infty$, then $f_{F D}(\lambda):=\chi_{\mathbb{R}_{-}}(\lambda), \lambda \in \mathbb{R}$. Notice that $\left[\rho^{e l}, p^{e l}\right]=0$. For $\rho^{p h}$ we choose the Gibbs state

$$
\rho^{p h}:=\frac{1}{Z} e^{-\beta h^{p h}}, \quad Z=\operatorname{tr}\left(e^{-\beta h^{p h}}\right)=\frac{1}{1-e^{-\beta \omega}},
$$

Hence $\rho^{p h}=\left(1-e^{-\beta \omega}\right) e^{-\beta h^{p h}}$. If $\beta=\infty$, then $\rho^{p h}:=\left(\cdot, \Upsilon_{0}\right) \Upsilon_{0}$. Obviously, $\operatorname{tr}\left(\rho^{p h}\right)=1$. We note that $\rho^{p h}(n)=\left(1-e^{-\beta \omega}\right) e^{-n \beta \omega}, n \in \mathbb{N}_{0}$, satisfies the condition (4.4) for any $p \geq 0$. Obviously, $\rho_{0}=\rho^{e l} \otimes \rho^{p h}$ is a density operator for $H_{0}$.

Definition 5.1 Let $\mathcal{S}=\left\{H, H_{0}\right\}$ be the $J C L$-model. If $Q:=Q_{\alpha}^{e l}$, where $Q_{\alpha}^{e l}$ is given by (5.1), and $\rho:=\rho_{0}:=\rho^{e l} \otimes \rho^{p h}$, where $\rho^{e l}$ and $\rho^{p h}$ are given by (5.2) and (5.3), then $J_{\rho_{0}, Q_{\alpha}^{e l}}^{e l}:=J_{\rho_{0}, Q_{\alpha}^{e l}}^{\mathcal{S}}$ is called the electron current entering the lead $\alpha$. The currents $J_{\rho_{0}, Q_{\alpha}^{e l}}^{c}$ and $J_{\rho_{0}, Q_{\alpha}^{e l}}^{p h}$ are called the contact induced and photon induced electron currents.

\subsubsection{Contact induced electron current}

The following proposition immediately follows from Proposition 4.2

Proposition 5.2 Let $S=\left\{H, H_{0}\right\}$ be the JCL-model. Then the contact induced electron current $J_{\rho_{0}, Q_{\alpha}^{e l}}^{c}, \alpha \in\{l, r\}$, is given by $J_{\rho_{0}, Q_{\alpha}^{e l}}^{c}=J_{\rho^{e l}, q_{\alpha}^{e l}}^{s_{c}}$. In particular, one has

$$
J_{\rho_{0}, Q_{\alpha}^{e l}}^{c}=-\frac{\mathfrak{e}}{2 \pi} \int_{\mathbb{R}}\left(f\left(\lambda-\mu_{\alpha}\right)-f\left(\lambda-\mu_{\varkappa}\right) \sigma_{c}(\lambda) d \lambda, \quad \alpha, \varkappa \in\{l, r\}, \quad \alpha \neq \varkappa,\right.
$$

where $\left\{\sigma_{c}(\lambda)\right\}_{\lambda \in \mathbb{R}}$ is the channel cross-section from left to the right of the scattering system $s_{c}=\left\{h^{\text {el }}, h_{0}^{e l}\right\}$, cf. Example 3.3 . 
Proof. Since $\operatorname{tr}\left(\rho^{p h}\right)=1$ it follows from Proposition 4.2 that $J_{\rho_{0}, Q_{\alpha}^{e l}}^{c}=J_{\rho^{e l}, q_{\alpha}^{e l}}^{s_{c}}$. From (3.26), cf. Example 3.3, we find (5.4).

If $\mu_{l}>\mu_{r}$ and $f(\cdot)$ is decreasing, then $J_{\rho_{0}, Q_{l}^{e l}}^{c}<0$. Hence the electron contact current is going from the left lead to the right which is in accordance with the physical intuition. In particular, this is valid for the Fermi-Dirac distribution.

Proposition 5.3 Let $\mathcal{S}=\left\{H, H_{0}\right\}$ be the JCL-model. Further, let $\rho^{e l}$ and $\rho^{p h}$ be given by (5.2) and (5.3), respectively. If the charge $Q_{\alpha}^{e l}$ is given by (5.1), then the following holds:

(E) If $\mu_{l}=\mu_{r}$, then $J_{\rho_{0}, Q_{\alpha}^{e l}}^{c}=0, \alpha \in\{l, r\}$.

(S) If $v_{l} \geq v_{r}+4$, then $J_{\rho_{0}, Q_{\alpha}^{e l}}^{c}=0, \alpha \in\{l, r\}$, even if $\mu_{l} \neq \mu_{l}$.

(C) If $e_{0}^{S}=\delta_{0}^{S}$ and $e_{1}^{S}=\delta_{1}^{S}$, then $J_{\rho_{0}, Q_{\alpha}^{e l}}^{c}=0, \alpha \in\{l, r\}$, even if $\mu_{l} \neq \mu_{l}$.

Proof. (E) If $\mu_{l}=\mu_{r}$, then $f\left(\lambda-\mu_{l}\right)=f\left(\lambda-\mu_{r}\right)$. Applying formula (5.4) we obtain $J_{\rho_{0}, Q_{\alpha}^{e l}}^{c}=0$.

(S) If $v_{l} \geq v_{r}+4$, then $h_{0}^{e l, a c}$ has simple spectrum. Hence the scattering matrix $\left\{s_{c}(\lambda)\right\}_{\lambda \in \mathbb{R}}$ of the scattering system $s_{c}=\left\{h^{e l}, h_{0}^{e l}\right\}$ is a scalar function which immediately yields $\sigma_{c}(\lambda)=0, \lambda \in \mathbb{R}$, which yields $J_{\rho_{0}, Q_{\alpha}^{e l}}^{c}=0$.

(C) In this case the Hamiltonian $h^{e l}$ decomposes into a direct sum of two Hamiltonians which do not interact. Hence the scattering matrix of $\left\{s_{c}(\lambda)\right\}_{\lambda \in \mathbb{R}}$ of the scattering system $s_{c}=\left\{h^{e l}, h_{0}^{e l}\right\}$ is diagonal which immediately yields $J_{\rho_{0}, Q_{\alpha}^{e l}}^{c}=0$.

\subsubsection{Photon induced electron current}

To analyze (4.12) is hopeless if we make no assumptions concerning $\rho^{e l}$ and the scattering operator $s_{c}$. The simplest assumptions is that $\rho^{e l}$ and $s_{c}$ commute. In this case we get $\widehat{\rho}^{e l}(\lambda)=\rho^{e l}(\lambda), \lambda \in \mathbb{R}$.

Lemma 5.4 Let $S=\left\{H, H_{0}\right\}$ be the JCL-model. Further let $\rho^{e l}$ be given by (5.2). If one of the cases $(E),(S)$ or $(C)$ of Proposition 5.3 is realized, then the $\rho^{\text {el }}$ and $s_{c}$ commute.

Proof. If $(E)$ holds, then $\rho^{e l}=f\left(h_{0}^{e l}\right)$ which yields $\left[\rho^{e l}, s_{c}\right]=0$. If $(S)$ is valid, then the scattering matrix $\left\{s_{c}(\lambda)\right\}_{\lambda \in \mathbb{R}}$ is a scalar function which shows $\left[\rho^{e l}, s_{c}\right]=0$. Finally, if $(C)$ is realized, then the scattering matrix $\left\{s_{c}(\lambda)\right\}_{\lambda \in \mathbb{R}}$ diagonal. Since the $\rho^{e l}$ is given by (5.2) we get $\left[\rho^{e l}, s_{c}\right]=0$.

We are going to calculate the current $J_{\rho_{0}, Q_{\alpha}^{\text {el }}}^{p h}$, see 4.12). Obviously, we have $P_{\alpha}(\lambda)=\sum_{n \in \mathbb{N}_{0}} p_{\alpha}^{e l}(\lambda-n \omega)$ and $I_{\mathfrak{h}(\lambda)}=P_{l}(\lambda)+P_{r}(\lambda), \lambda \in \mathbb{R}$. We set

$$
P_{n_{\alpha}}(\lambda):=P_{\alpha}(\lambda) P_{n}(\lambda)=P_{n}(\lambda) P_{\alpha}(\lambda)=p_{\alpha}^{e l}(\lambda-n \omega), \alpha \in\{l, r\},
$$


$n \in \mathbb{N}_{0}, \lambda \in \mathbb{R}$. In the following we use the notation $\widehat{T}_{p h}(\lambda)=\widehat{S}_{p h}(\lambda)-I_{\mathfrak{h}(\lambda)}$, $\lambda \in \mathbb{R}$, where $\left\{\widehat{T}_{p h}(\lambda)\right\}_{\lambda \in \mathbb{R}}$ is called the transition matrix and $\left\{\widehat{S}_{p h}(\lambda)\right\}_{\lambda \in \mathbb{R}}$ is given by (3.28). We set

$$
\widehat{T}_{k_{\alpha} m_{\varkappa}}^{p h}(\lambda):=P_{k_{\alpha}}(\lambda) \widehat{T}_{p h}(\lambda) P_{m_{\varkappa}}(\lambda), \quad \lambda \in \mathbb{R}, \quad \alpha, \varkappa \in\{l, r\}, \quad k, m \in \mathbb{N}_{0} .
$$

and

$$
\widehat{\sigma}_{k_{\alpha} m_{\varkappa}}^{p h}(\lambda)=\operatorname{tr}\left(\widehat{T}_{k_{\alpha} m_{\varkappa}}^{p h}(\lambda)^{*} \widehat{T}_{k_{\alpha} m_{\varkappa}}^{p h}(\lambda)\right), \quad \lambda \in \mathbb{R},
$$

which is the cross-section between the channels $k_{\alpha}$ and $m_{\varkappa}$.

Proposition 5.5 Let $\mathcal{S}=\left\{H, H_{0}\right\}$ be the JCL-model.

(i) If $\rho^{\text {el }}$ commutes with the scattering operator $s_{c}$ and $q^{e l}$, then

$$
\begin{aligned}
& J_{\rho_{0}, Q_{\alpha}^{e l}}^{p h}=-\sum_{\substack{m, n \in \mathbb{N}_{0} \\
\varkappa \in\{l, r\}}} \frac{\mathfrak{e}}{2 \pi} \int_{\mathbb{R}} \times \\
& \left(\rho^{p h}(n) f\left(\lambda-\mu_{\alpha}-n \omega\right)-\rho^{p h}(m) f\left(\lambda-\mu_{\varkappa}-m \omega\right)\right) \widehat{\sigma}_{n_{\alpha} m_{\varkappa}}^{p h}(\lambda) d \lambda .
\end{aligned}
$$

(ii) If in addition $\mathcal{S}=\left\{H, H_{0}\right\}$ is time reversible symmetric, then

$$
\begin{gathered}
J_{\rho_{0}, Q_{\alpha}^{e l}}^{p h}=-\sum_{m, n \in \mathbb{N}_{0}} \frac{\mathfrak{e}}{2 \pi} \int_{\mathbb{R}} \times \\
\left(\rho^{p h}(n) f\left(\lambda-\mu_{\alpha}-n \omega\right)-\rho^{p h}(m) f\left(\lambda-\mu_{\alpha^{\prime}}-m \omega\right)\right) \widehat{\sigma}_{n_{\alpha} m_{\alpha^{\prime}}}^{p h}(\lambda) d \lambda, \\
\alpha, \alpha^{\prime} \in\{l, r\}, \alpha \neq \alpha^{\prime} .
\end{gathered}
$$

Proof. (i) Let us assume that

$$
q^{e l}=\sum_{\varkappa \in\{l, r\}} g_{\varkappa}\left(h_{\varkappa}^{e l}\right),
$$

Notice that

$$
q_{a c}^{e l}(\lambda)=\sum_{\varkappa \in\{l, r\}} g_{\varkappa}(\lambda) p_{\varkappa}^{e l}(\lambda), \quad \lambda \in \mathbb{R} .
$$

Inserting (5.8) into 4.12) and using $q^{p h}=I_{\mathfrak{h}^{p h}}$ we get

$$
\begin{aligned}
J_{\rho_{0}, Q}^{p h}= & \sum_{\substack{m \in \mathbb{N}_{0} \\
\alpha \in\{l, r\}}} \rho^{p h}(m) \sum_{\substack{n \in \mathbb{N}_{0} \\
\varkappa \in\{l, r\}}} \frac{1}{2 \pi} \int_{\mathbb{R}} d \lambda \phi_{\alpha}(\lambda-m \omega) g_{\varkappa}(\lambda-n \omega) \times \\
& \operatorname{tr}\left(p_{\alpha}^{e l}(\lambda-m \omega)\left(p_{\varkappa}^{e l}(\lambda-n \omega) \delta_{m n}-\widehat{S}_{n m}^{p h}(\lambda)^{*} p_{\varkappa}^{e l}(\lambda-n \omega) \widehat{S}_{n m}^{p h}(\lambda)\right)\right)
\end{aligned}
$$

where for simplicity we have set

$$
\phi_{\alpha}(\lambda):=f\left(\lambda-\mu_{\alpha}\right), \quad \lambda \in \mathbb{R}, \quad n \in \mathbb{N}_{0}, \quad \alpha \in\{l, r\} .
$$


Obviously, we have

$$
\begin{aligned}
J_{\rho_{0}, Q}^{p h}= & \sum_{\substack{n \in \mathbb{N}_{0} \\
\varkappa \in\{l, r\}}} \rho^{p h}(n) \frac{1}{2 \pi} \int_{\mathbb{R}} d \lambda \phi_{\varkappa}(\lambda-n \omega) g_{\varkappa}(\lambda-n \omega) \operatorname{tr}\left(p_{\varkappa}^{e l}(\lambda-n \omega)\right)- \\
& \sum_{\substack{n \in \mathbb{N}_{0}, \varkappa \in\{l, r\}}} \sum_{\substack{m \in \mathbb{N}_{0} \\
\alpha \in\{l, r\}}} \rho^{p h}(m) \frac{1}{2 \pi} \int_{\mathbb{R}} d \lambda \phi_{\alpha}(\lambda-m \omega) g_{\varkappa}(\lambda-n \omega) \times \\
& \operatorname{tr}\left(p_{\alpha}^{e l}(\lambda-m \omega) \widehat{S}_{n m}^{p h}(\lambda)^{*} p_{\varkappa}^{e l}(\lambda-n \omega) \widehat{S}_{n m}^{p h}(\lambda) p_{\alpha}^{e l}(\lambda-m \omega)\right) .
\end{aligned}
$$

Since the scattering matrix $\left\{\widehat{S}^{p h}(\lambda)\right\}_{\lambda \in \mathbb{R}}$ is unitary we have

$$
p_{\varkappa}^{e l}(\lambda-n \omega)=\sum_{\substack{m \in \mathbb{N}_{0} \\ \alpha \in\{l, r\}}} p_{\varkappa}^{e l}(\lambda-n \omega) \widehat{S}_{m n}^{p h}(\lambda)^{*} p_{\alpha}^{e l}(\lambda-m \omega) \widehat{S}_{m n}^{p h}(\lambda) p_{\varkappa}^{e l}(\lambda-n \omega)
$$

for $n \in \mathbb{N}_{0}$ and $\varkappa \in\{l, r\}$. Inserting (5.11) into (5.10) we find

$$
\begin{aligned}
J_{\rho_{0}, Q}^{p h}= & \sum_{\substack{n \in \mathbb{N}_{0} \\
\varkappa \in\{l, r\}}} \sum_{\substack{m \in \mathbb{N}_{0} \\
\alpha \in\{l, r\}}} \rho^{p h}(n) \frac{1}{2 \pi} \int_{\mathbb{R}} d \lambda \phi_{\varkappa}(\lambda-n \omega) g_{\varkappa}(\lambda-n \omega) \times \\
& \operatorname{tr}\left(p_{\varkappa}^{e l}(\lambda-n \omega) \widehat{S}_{n m}^{p h}(\lambda)^{*} p_{\alpha}^{e l}(\lambda-m \omega) \widehat{S}_{m n}^{p h}(\lambda) p_{\varkappa}^{e l}(\lambda-n \omega)\right)- \\
& \sum_{\substack{n \in \mathbb{N}_{0} \\
\varkappa \in\{l, r\}}} \sum_{\substack{m \in \mathbb{N}_{0} \\
\alpha \in\{l, r\}}} \rho^{p h}(m) \frac{1}{2 \pi} \int_{\mathbb{R}} d \lambda \phi_{\alpha}(\lambda-m \omega) g_{\varkappa}(\lambda-n \omega) \times \\
& \operatorname{tr}\left(p_{\alpha}^{e l}(\lambda-m \omega) \widehat{S}_{n m}^{p h}(\lambda)^{*} p_{\varkappa}^{e l}(\lambda-n \omega) \widehat{S}_{n m}^{p h}(\lambda) p_{\alpha}^{e l}(\lambda-m \omega)\right) .
\end{aligned}
$$

Using the notation (5.5) we find

$$
\begin{aligned}
J_{\rho_{0}, Q}^{p h}= & \sum_{\substack{n \in \mathbb{N}_{0} \\
\varkappa \in\{l, r\}}} \sum_{\substack{m \in \mathbb{N}_{0} \\
\alpha \in\{l, r\}}} \rho^{p h}(n) \frac{1}{2 \pi} \int_{\mathbb{R}} d \lambda \phi_{\varkappa}(\lambda-n \omega) g_{\varkappa}(\lambda-n \omega) \widehat{\sigma}_{m_{\alpha} n_{\varkappa}}^{p h}(\lambda)- \\
& \sum_{\substack{n \in \mathbb{N}_{0} \\
\varkappa \in\{l, r\}}} \sum_{\substack{m \in \mathbb{N}_{0} \\
\alpha \in\{l, r\}}} \rho^{p h}(m) \frac{1}{2 \pi} \int_{\mathbb{R}} d \lambda \phi_{\alpha}(\lambda-m \omega) g_{\varkappa}(\lambda-n \omega) \widehat{\sigma}_{n_{\varkappa}}^{p h}(\lambda):
\end{aligned}
$$

By 3.16 we find

$$
\sum_{\substack{m \in \mathbb{N}_{0} \\ \alpha \in\{l, r\}}} \widehat{\sigma}_{m_{\alpha} n_{\varkappa}}^{p h}(\lambda)=\sum_{\substack{m \in \mathbb{N}_{0} \\ \alpha \in\{l, r\}}} \widehat{\sigma}_{n_{\varkappa} m_{\alpha}}^{p h}(\lambda) \quad \lambda \in \mathbb{R} .
$$

Using that we get

$$
\begin{aligned}
& J_{\rho_{0}, Q}^{p h}=\sum_{\substack{m, n \in \mathbb{N}_{0} \\
\alpha, \varkappa \in\{l, r\}}} \frac{1}{2 \pi} \int_{\mathbb{R}} \times \\
& \left(\rho^{p h}(n) \phi_{\varkappa}(\lambda-n \omega)-\rho^{p h}(m) \phi_{\alpha}(\lambda-m \omega)\right) g_{\varkappa}(\lambda-n \omega) \widehat{\sigma}_{n_{\varkappa} m_{\alpha}}^{p h}(\lambda) d \lambda .
\end{aligned}
$$


Setting $g_{\alpha}(\lambda)=-\mathfrak{e}$ and $g_{\varkappa}(\lambda) \equiv 0, \varkappa \neq \alpha$, we obtain (5.6).

(ii) A straightforward computation shows that

$$
\begin{gathered}
\sum_{n, m \in \mathbb{N}_{0}} \int_{\mathbb{R}}\left(\rho^{p h}(n) f\left(\lambda-\mu_{a}-n \omega\right)-\rho^{p h}(m) f\left(\lambda-\mu_{a}-m \omega\right)\right) \widehat{\sigma}_{n_{\alpha} m_{\alpha}}^{p h}(\lambda) d \lambda= \\
\sum_{n, m \in \mathbb{N}_{0}} \int_{\mathbb{R}}\left(\rho^{p h}(m) f\left(\lambda-\mu_{a}-m \omega\right)-\rho^{p h}(n) f\left(\lambda-\mu_{a}-n \omega\right)\right) \widehat{\sigma}_{m_{\alpha} n_{\alpha}}^{p h}(\lambda) d \lambda
\end{gathered}
$$

Since $\sigma_{m_{\alpha} n_{\alpha}}^{p h}(\lambda)=\sigma_{n_{\alpha} m_{\alpha}}^{p h}(\lambda), \lambda \in \mathbb{R}$, we get

$$
\begin{aligned}
& \sum_{n, m \in \mathbb{N}_{0}} \int_{\mathbb{R}}\left(\rho^{p h}(n) f\left(\lambda-\mu_{a}-n \omega\right)-\rho^{p h}(m) f\left(\lambda-\mu_{a}-m \omega\right)\right) \widehat{\sigma}_{n_{\alpha}}^{p h} m_{\alpha}(\lambda) d \lambda= \\
& -\sum_{n, m \in \mathbb{N}_{0}} \int_{\mathbb{R}}\left(\rho^{p h}(n) f\left(\lambda-\mu_{a}-n \omega\right)-\rho^{p h}(m) f\left(\lambda-\mu_{a}-m \omega\right)\right) \widehat{\sigma}_{n_{\alpha}}^{p h} m_{\alpha}(\lambda) d \lambda
\end{aligned}
$$

which yields

$$
\sum_{n, m \in \mathbb{N}_{0}} \int_{\mathbb{R}}\left(\rho^{p h}(n) f\left(\lambda-\mu_{a}-n \omega\right)-\rho^{p h}(m) f\left(\lambda-\mu_{a}-m \omega\right)\right) \widehat{\sigma}_{n_{\alpha}}^{p h} m_{\alpha}(\lambda) d \lambda=0 .
$$

Using that we get immediately the representation (5.7) from (5.6).

Corollary 5.6 Let $\mathcal{S}=\left\{H, H_{0}\right\}$ be the JCL-model.

(i) If the cases cases $(E),(S)$ or $(C)$ of Proposition 5.3 are realized, then the representation (5.6) holds.

(ii) If the case (E) of Proposition 5.3 is realized and the system $\mathcal{S}=\left\{H, H_{0}\right\}$ is time reversible symmetric, then

$$
\begin{aligned}
& J_{\rho_{0}, Q_{\alpha}^{e l}}^{p h}= \\
& -\sum_{m, n \in \mathbb{N}_{0}} \frac{\mathfrak{e}}{2 \pi} \int_{\mathbb{R}}\left(\rho^{p h}(n) f(\lambda-\mu-n \omega)-\rho^{p h}(m) f(\lambda-\mu-m \omega)\right) \widehat{\sigma}_{n_{\alpha} m_{\alpha^{\prime}}}^{p h}(\lambda) d \lambda
\end{aligned}
$$

$n \in \mathbb{N}_{0}, \alpha \in\{l, r\}$ where $\mu:=\mu_{l}=\mu_{r}$ and $\alpha \neq \alpha^{\prime}$.

(iii) If the case (E) of Proposition 5.3 is realized and the system $\mathcal{S}=\left\{H, H_{0}\right\}$ is time reversible and mirror symmetric, then $J_{\rho_{0}, Q_{\alpha}^{e l}}^{p h}=0$.

Proof. (i) The statement follows from Proposition 5.5 (i) and Lemma 5.4

(ii) Setting $\mu_{\alpha}=\mu_{\alpha^{\prime}}$ formula (5.13) follows (5.7).

(iii) If $\mathcal{S}=\left\{H, H_{0}\right\}$ is time reversible and mirror symmetric we get from Lemma 2.14(ii) that $\widehat{\sigma}_{n_{\alpha} m_{\alpha^{\prime}}}^{p h}(\lambda)=\widehat{\sigma}_{n_{\alpha^{\prime}} m_{\alpha}}^{p h}(\lambda), \lambda \in \mathbb{R}, n, m \in \mathbb{N}_{0}, \alpha, \alpha^{\prime} \in\{l, r\}, \alpha \neq \alpha^{\prime}$. Using that we get from (5.13) that

$$
\begin{aligned}
& J_{\rho_{0}, Q_{\alpha}^{e l}}^{p h}= \\
& -\sum_{m, n \in \mathbb{N}_{0}} \frac{\mathfrak{e}}{2 \pi} \int_{\mathbb{R}}\left(\rho^{p h}(n) f(\lambda-\mu-n \omega)-\rho^{p h}(m) f(\lambda-\mu-m \omega)\right) \widehat{\sigma}_{n_{\alpha^{\prime}} m_{\alpha}}^{p h}(\lambda) d \lambda .
\end{aligned}
$$


Interchanging $m$ and $n$ we get

$$
\begin{aligned}
& J_{\rho_{0}, Q_{\alpha}^{e l}}^{p h}= \\
& -\sum_{m, n \in \mathbb{N}_{0}} \frac{\mathfrak{e}}{2 \pi} \int_{\mathbb{R}}\left(\rho^{p h}(m) f(\lambda-\mu-m \omega)-\rho^{p h}(n) f(\lambda-\mu-n \omega)\right) \widehat{\sigma}_{m_{\alpha^{\prime}} n_{\alpha}}^{p h}(\lambda) d \lambda .
\end{aligned}
$$

Using that $S$ is time reversible symmetric we get from Lemma2.14(i) that

$$
\begin{aligned}
& J_{\rho_{0}, Q_{\alpha}^{e l}}^{p h}= \\
& -\sum_{m, n \in \mathbb{N}_{0}} \frac{\mathfrak{e}}{2 \pi} \int_{\mathbb{R}}\left(\rho^{p h}(m) f(\lambda-\mu-m \omega)-\rho^{p h}(n) f(\lambda-\mu-n \omega)\right) \widehat{\sigma}_{n_{\alpha} m_{\alpha^{\prime}}}^{p h}(\lambda) d \lambda .
\end{aligned}
$$

which shows that $J_{\rho_{0}, Q_{\alpha}^{e l}}^{p h}=-J_{\rho_{0}, Q_{\alpha}^{e l}}^{p h}$. Hence $J_{\rho_{0}, Q_{\alpha}^{e l}}^{p h}=0$.

We note that by Proposition 5.3 the contact induced current is zero, i.e. $J_{\rho_{0}, Q_{\alpha}^{e l}}^{c}=0$. Hence, if the $\mathcal{S}$ is time reversible and mirror symmetric, then the total current is zero, i.e. $J_{\rho_{0}, Q_{\alpha}^{e l}}^{\mathcal{S}}=0$.

Remark 5.7 Let the case $(E)$ of Proposition 5.3 be realized, that is, $\mu_{l}=\mu_{r}$. Moreover, we assume for simplicity that $0=: v_{r} \leq v:=v_{l}$.

(i) If $\beta=\infty$, then $\rho^{p h}(n)=\delta_{0 n}, n \in \mathbb{N}_{0}$. From (5.6) we immediately get that $J_{\rho^{e l}, Q_{\alpha}^{e l}}^{p h}=0$. That means, if the temperature is zero, then the photon induced electron current is zero.

(ii) The photon induced electron current might be zero even if $\beta<\infty$. Indeed, let $\mathcal{S}=\left\{H, H_{0}\right\}$ be time reversible symmetric and let the case (E) be realized. If $\omega \geq v+4$ and, then $\mathfrak{h}^{e l}(\lambda):=\mathfrak{h}_{n}^{e l}(\lambda)=\mathfrak{h}^{e l}(\lambda-n \omega), n \in \mathbb{N}_{0}$. Hence one always has $n=m$ in formula (5.13) which immediately yields $J_{\rho_{0}, Q_{\alpha}^{e l}}^{p h}=0$.

(iii) The photon induced electron current might be different from zero. Indeed, let $\mathcal{S}=\left\{H, H_{0}\right\}$ be time reversible symmetric and let $v=2$ and $\omega=4$, then one gets that to calculate the $J_{\rho_{0}, Q_{l}^{e l}}^{p h}$ one has to take into account $m=n+1$ in formula (5.13). Therefore we find

$$
\begin{aligned}
& J_{\rho_{0}, Q_{l}^{e l}}^{p h}=-\sum_{n \in \mathbb{N}_{0}} \frac{\mathfrak{e}}{2 \pi} \int_{\mathbb{R}} d \lambda \times \\
& \left(\rho^{p h}(n) f(\lambda-\mu-n \omega)-\rho^{p h}(n+1) f(\lambda-\mu-(n+1) \omega)\right) \widehat{\sigma}_{n_{l}(n+1)_{r}}^{p h}(\lambda) .
\end{aligned}
$$

If $\rho^{p h}$ is given by (5.3) and $f(\lambda)=f_{F D}(\lambda)$, cf. [3.21], then one easily verifies that

$$
\frac{\partial}{\partial x} \rho^{p h}(x) f_{F D}(\lambda-\mu-x \omega)<0, \quad x, \mu, \lambda \in \mathbb{R} .
$$

Hence $\rho^{p h}(n) f_{F D}(\lambda-\mu-n \omega)$ is decreasing in $n \in \mathbb{N}_{0}$ for $\lambda, \mu \in \mathbb{R}$ which yields $\left(\rho^{p h}(n) f(\lambda-\mu-n \omega)-\rho^{p h}(n+1) f(\lambda-\mu-(n+1) \omega)\right) \geq 0$. 
Therefore $J_{\rho_{0}, Q_{l}^{e l}}^{p h} \leq 0$ which means that the photon induced current leaves the left-hand side and enters the right-hand side. In fact $J_{\rho_{0}, Q_{l}^{e l}}^{p h}=0$ implies that $\widehat{\sigma}_{n_{l}(n+1)_{n}}^{p h}(\lambda)=0$ for $n \in \mathbb{N}_{0}$ and $\lambda \in \mathbb{R}$ which means that there is no scattering from the left-hand side to the right one and vice versa which can be excluded generically.

\subsection{Photon current}

The photon current is related to the charge

$$
Q:=Q^{p h}=-I_{\mathfrak{h} e l} \otimes \mathfrak{n},
$$

where $\mathfrak{n}=\mathrm{d} \Gamma(1)=b^{*} b$ is the photon number operator on $\mathfrak{h}^{p h}=\mathfrak{F}_{+}(\mathbb{C})$, which is selfadjoint and commutes with $h^{p h}$. It follows that $Q^{p h}$ is also self-adjoint and commutes with $H_{0}$. It is not bounded, but since $\operatorname{dom}(\mathfrak{n})=\operatorname{dom}\left(h^{p h}\right)$, it is immediately obvious that $Q^{p h}\left(H_{0}+\theta\right)^{-1}$ is bounded, whence $\mathfrak{N}$ is a tempered charge. Its charge matrix with respect to the spectral representation $\Pi\left(H_{0}^{a c}\right)$ of Lemma2.12 is given by

$$
Q_{a c}^{p h}(\lambda)=-\bigoplus_{n \in \mathbb{N}_{0}} n P_{n}(\lambda)
$$

We recall that $P_{n}(\lambda)$ is the orthogonal projection form $\mathfrak{h}(\lambda)$ onto $\mathfrak{h}_{n}(\lambda)=\mathfrak{h}^{e l}(\lambda-n \omega)$, $\lambda \in \mathbb{R}$. We are going to calculate the photon current or, how it is also called, the photon production rate.

\subsubsection{Contact induced photon current}

The following proposition is in accordance with the physical intuition.

Proposition 5.8 Let $\mathcal{S}=\left\{H, H_{0}\right\}$ be the JCL-model. Then $J_{\rho_{0}, Q^{p h}}^{c}=0$.

Proof. We note that $q_{a c}^{e l}(\lambda)=I_{\mathfrak{h} e l(\lambda)}, \lambda \in \mathbb{R}$. Inserting this into (3.25) we get $J_{\rho^{e l}, q^{e l}}^{s_{c}}=0$. Applying Proposition 4.2 we prove $J_{\rho_{0}, Q^{p h}}^{c}=0$.

The result reflects the fact that the lead contact does not contributed to the photon current which is plausible.

\subsubsection{Photon current}

From the Proposition 5.8 we get that only the photon induced photon current $J_{\rho_{0}, Q^{p h}}^{p h}$ contributes to the photon current $J_{\rho_{0}, Q^{p h}}^{\mathcal{S}}$. Since $J_{\rho_{0}, Q^{p h}}^{\mathcal{S}}=J_{\rho_{0}, Q^{p h}}^{p h}$ we call $J_{\rho_{0}, Q^{p h}}^{p h}$ simply the photon current.

We set

Using the notation $\widehat{T}_{n m}^{p h}(\lambda):=P_{n}(\lambda) \widehat{T}_{p h}(\lambda)\left\lceil\mathfrak{h}^{e l}(\lambda-m \omega), \lambda \in \mathbb{R}, m, n \in \mathbb{N}_{0}\right.$.

$$
\widetilde{T}_{n m}^{p h}(\lambda)=\widehat{T}_{n m}^{p h}(\lambda) s_{c}(\lambda-m \omega), \quad \lambda \in \mathbb{R}, \quad m, n \in \mathbb{N}_{0}
$$


and

$$
\widetilde{T}_{n_{\varkappa} m_{\alpha}}^{p h}(\lambda):=P_{n_{\varkappa}}(\lambda) \widetilde{T}_{n m}^{p h}(\lambda)\left\lceil\mathfrak{h}_{\alpha}^{e l}(\lambda-m \omega), \quad \lambda \in \mathbb{R},\right.
$$

$m, n \in \mathbb{N}_{0}, \alpha, \varkappa \in\{l, r\}$, as well as $\widetilde{\sigma}_{n_{\varkappa} m_{\alpha}}^{p h}(\lambda):=\operatorname{tr}\left(\widetilde{T}_{n_{\varkappa} m_{\alpha}}^{p h}(\lambda)^{*} \widetilde{T}_{n_{\varkappa} m_{\alpha}}^{p h}(\lambda)\right), \lambda \in \mathbb{R}$.

Proposition 5.9 Let $\mathcal{S}=\left\{H, H_{0}\right\}$ be the JCL-model.

(i) Then

$$
J_{\rho_{0}, Q^{p h}}^{p h}=\sum_{\substack{m, n \in \mathbb{N}_{0} \\ \alpha, \varkappa \in\{l, r\}}}(n-m) \rho^{p h}(m) \frac{1}{2 \pi} \int_{\mathbb{R}} f\left(\lambda-\mu_{\alpha}-m \omega\right) \widetilde{\sigma}_{n_{\varkappa} m_{\alpha}}^{p h}(\lambda) d \lambda
$$

(ii) If $\rho^{e l}$ commutes with $s_{c}$, then

$$
J_{\rho_{0}, Q^{p h}}^{p h}=\sum_{\substack{m, n \in \mathbb{N}_{0} \\ \alpha, \varkappa \in\{l, r\}}}(n-m) \rho^{p h}(m) \frac{1}{2 \pi} \int_{\mathbb{R}} f\left(\lambda-\mu_{\alpha}-m \omega\right) \widehat{\sigma}_{n_{\varkappa} m_{\alpha}}^{p h}(\lambda) d \lambda
$$

(iii) If $\rho^{e l}$ commutes with $s_{c}$ and $S=\left\{H, H_{0}\right\}$ is time reversible symmetric, then

$$
\begin{aligned}
& J_{\rho_{0}, Q^{p h}}^{p h}=\sum_{\substack{m, n \in \mathbb{N}_{0}, n>m \\
\varkappa, \alpha \in\{l, r\}}} \frac{1}{2 \pi} \int_{\mathbb{R}} d \lambda \times \\
& (n-m)\left(\rho^{p h}(m) f\left(\lambda-\mu_{\alpha}-m \omega\right)-\rho^{p h}(n) f\left(\lambda-\mu_{\varkappa}-n \omega\right)\right) \widehat{\sigma}_{n_{\varkappa} m_{\alpha}}^{p h}(\lambda)
\end{aligned}
$$

where $\alpha^{\prime} \in\{l, r\}$ and $\alpha^{\prime} \neq \alpha$.

Proof. (i) From (4.12) we get

$$
\begin{aligned}
J_{\rho_{0}, Q^{p h}}^{p h}= & -\sum_{m, n \in \mathbb{N}_{0}} n \rho^{p h}(m) \frac{1}{2 \pi} \int_{\mathbb{R}} d \lambda \operatorname{tr}\left(\widehat{\rho}_{a c}^{e l}(\lambda-m \omega) \times\right. \\
& \left.\left(P_{n}(\lambda) \delta_{m n}-\widehat{S}_{n m}^{p h}(\lambda)^{*} q_{a c}^{e l}(\lambda-n \omega) \widehat{S}_{n m}^{p h}(\lambda)\right)\right) .
\end{aligned}
$$

Hence

$$
\begin{aligned}
J_{\rho_{0}, Q^{p h}}^{p h} & =-\sum_{m \in \mathbb{N}_{0}} m \rho^{p h}(m) \times \\
& \frac{1}{2 \pi} \int_{\mathbb{R}} \operatorname{tr}\left(\widehat{\rho}_{a c}^{e l}(\lambda-m \omega)\left(P_{m}(\lambda)-\widehat{S}_{m m}^{p h}(\lambda)^{*} P_{m}(\lambda) \widehat{S}_{m m}^{p h}(\lambda)\right)\right) d \lambda+ \\
& \sum_{\substack{m, n \in \mathbb{N}_{0} \\
m \neq n}} n \rho^{p h}(m) \frac{1}{2 \pi} \int_{\mathbb{R}} \operatorname{tr}\left(\widehat{\rho}_{a c}^{e l}(\lambda-m \omega) \widehat{S}_{n m}^{p h}(\lambda)^{*} P_{n}(\lambda) \widehat{S}_{n m}^{p h}(\lambda)\right) d \lambda .
\end{aligned}
$$


Using the relation $P_{m}(\lambda)=I_{\mathfrak{h}(\lambda)}-\sum_{n \in \mathbb{N}_{0}, m \neq n} P_{n}(\lambda), \lambda \in \mathbb{R}$, we get

$$
\begin{aligned}
& J_{\rho_{0}, Q^{p h}}^{p h}= \\
& -\sum_{\substack{m, n \in \mathbb{N}_{0} \\
m \neq n}} m \rho^{p h}(m) \frac{1}{2 \pi} \int_{\mathbb{R}} \operatorname{tr}\left(\widehat{\rho}_{a c}^{e l}(\lambda-m \omega)\left(\widehat{S}_{n m}^{p h}(\lambda)^{*} P_{n}(\lambda) \widehat{S}_{n m}^{p h}(\lambda)\right)\right) d \lambda+ \\
& \sum_{\substack{m, n \in \mathbb{N}_{0} \\
m \neq n}} n \rho^{p h}(m) \frac{1}{2 \pi} \int_{\mathbb{R}} \operatorname{tr}\left(\widehat{\rho}_{a c}^{e l}(\lambda-m \omega) \widehat{S}_{n m}^{p h}(\lambda)^{*} P_{n}(\lambda) \widehat{S}_{n m}^{p h}(\lambda)\right) d \lambda .
\end{aligned}
$$

Since $\widehat{T}_{p h}(\lambda)=\widehat{S}_{p h}(\lambda)-I_{\mathfrak{h}(\lambda)}, \lambda \in \mathbb{R}$, we find

$$
\begin{aligned}
& J_{\rho_{0}, Q^{p h}}^{p h}= \\
& -\sum_{m, n \in \mathbb{N}_{0}}(m-n) \rho^{p h}(m) \frac{1}{2 \pi} \int_{\mathbb{R}} \operatorname{tr}\left(\widehat{\rho}_{a c}^{e l}(\lambda-m \omega) \widehat{T}_{n m}^{p h}(\lambda)^{*} \widehat{T}_{n m}^{p h}(\lambda)\right) d \lambda .
\end{aligned}
$$

Using (4.13) and definition (5.14) one gets

$$
\begin{aligned}
& J_{\rho_{0}, Q^{p h}}^{p h}= \\
& -\sum_{m, n \in \mathbb{N}_{0}}(m-n) \rho^{p h}(m) \frac{1}{2 \pi} \int_{\mathbb{R}} \operatorname{tr}\left(\rho_{a c}^{e l}(\lambda-m \omega) \widetilde{T}_{n m}^{p h}(\lambda)^{*} \widetilde{T}_{n m}^{p h}(\lambda)\right) d \lambda .
\end{aligned}
$$

Since $\rho_{a c}^{e l}=\rho_{l}^{e l} \oplus \rho_{r}^{e l}$ where $\rho_{\alpha}^{e l}$ is given by (5.2) we find

$$
\begin{aligned}
& J_{\rho_{0}, Q^{p h}}^{p h}= \\
& -\sum_{\substack{m, n \in \mathbb{N}_{0} \\
\alpha, \varkappa \in\{l, r\}}}(m-n) \rho^{p h}(m) \frac{1}{2 \pi} \int_{\mathbb{R}} f\left(\lambda-\mu_{\alpha}-m \omega\right) \operatorname{tr}\left(\widetilde{T}_{n_{\varkappa} m_{\alpha}}^{p h}(\lambda)^{*} \widetilde{T}_{n_{\varkappa} m_{\alpha}}^{p h}(\lambda)\right) d \lambda
\end{aligned}
$$

where we have used (5.15). Using $\widetilde{\sigma}_{n_{\varkappa} m_{\alpha}}^{p h}(\lambda)=\operatorname{tr}\left(\widetilde{T}_{n_{\varkappa} m_{\alpha}}^{p h}(\lambda) * \widetilde{T}_{n_{\varkappa} m_{\alpha}}^{p h}(\lambda)\right)$ we prove (5.16.

(ii) If $\rho_{a c}^{e l}$ commutes with $s_{c}$, then $\widehat{\rho}_{a c}^{e l}(\lambda)=\rho_{a c}^{e l}(\lambda), \lambda \in \mathbb{R}$ which yields that one can replace $\widetilde{\sigma}_{n_{\varkappa} m_{\alpha}}^{p h}(\lambda)$ by $\widehat{\sigma}_{n_{\varkappa} m_{\alpha}}^{p h}(\lambda), \lambda \in \mathbb{R}$. Therefore (5.17) holds.

(iii) Obviously we have

$$
\begin{aligned}
& J_{\rho_{0}, Q^{p h}}^{p h}= \\
& \sum_{\substack{m, n \in \mathbb{N}_{0}, n>m \\
\alpha, \varkappa \in\{l, r\}}}(n-m) \rho^{p h}(m) \frac{1}{2 \pi} \int_{\mathbb{R}} f\left(\lambda-\mu_{\alpha}-m \omega\right) \widehat{\sigma}_{n_{\varkappa} m_{\alpha}}^{p h}(\lambda) d \lambda+ \\
& \sum_{\substack{m, n \in \mathbb{N}_{0}, n<m \\
\alpha, \varkappa \in\{l, r\}}}(n-m) \rho^{p h}(m) \frac{1}{2 \pi} \int_{\mathbb{R}} f\left(\lambda-\mu_{\alpha}-m \omega\right) \widehat{\sigma}_{n_{\varkappa} m_{\alpha}}^{p h}(\lambda) d \lambda .
\end{aligned}
$$


Moreover, a straightforward computation shows that

$$
\begin{aligned}
& \sum_{\substack{m, n \in \mathbb{N}_{0}, n<m \\
\alpha, \varkappa \in\{l, r\}}}(n-m) \rho^{p h}(m) \frac{1}{2 \pi} \int_{\mathbb{R}} f\left(\lambda-\mu_{\alpha}-m \omega\right) \widehat{\sigma}_{n_{\varkappa} m_{\alpha}}^{p h}(\lambda) d \lambda= \\
& \sum_{\substack{m, n \in \mathbb{N}_{0}, n>m \\
\alpha, \varkappa \in\{l, r\}}}(m-n) \rho^{p h}(n) \frac{1}{2 \pi} \int_{\mathbb{R}} f\left(\lambda-\mu_{\varkappa}-n \omega\right) \widehat{\sigma}_{m_{\alpha} n_{\varkappa}}^{p h}(\lambda) d \lambda .
\end{aligned}
$$

Since $\mathcal{S}=\left\{H, H_{0}\right\}$ is time reversible symmetric we find

$$
\begin{aligned}
& \sum_{\substack{m, n \in \mathbb{N}_{0}, n<m \\
\alpha, \varkappa \in\{l, r\}}}(n-m) \rho^{p h}(m) \frac{1}{2 \pi} \int_{\mathbb{R}} f\left(\lambda-\mu_{\alpha}-m \omega\right) \widehat{\sigma}_{m_{\alpha}}^{p h} n_{\varkappa}(\lambda) d \lambda= \\
& \sum_{\substack{m, n \in \mathbb{N}_{0}, n>m \\
\alpha, \varkappa \in\{l, r\}}}(m-n) \rho^{p h}(n) \frac{1}{2 \pi} \int_{\mathbb{R}} f\left(\lambda-\mu_{\varkappa}-n \omega\right) \widehat{\sigma}_{n_{\varkappa} m_{\alpha}}^{p h}(\lambda) d \lambda .
\end{aligned}
$$

Inserting 5.20 into 5.19) we obtain 5.18).

Corollary 5.10 Let $\mathcal{S}=\left\{H, H_{0}\right\}$ be the JCL-model and let $f=f_{F D}$. If case $(E)$ of Proposition 5.3 is realized and $\mathcal{S}=\left\{H, H_{0}\right\}$ is time reversible symmetric, then $J_{\rho_{0}, Q^{p h}}^{p h} \geq 0$.

Proof. We set $\mu:=\mu_{l}=\mu_{r}$. One has

$$
\begin{aligned}
& \rho^{p h}(m) f(\lambda-\mu-m \omega)-\rho^{p h}(n) f(\lambda-\mu-n \omega)= \\
& \quad e^{-m \beta \omega}\left(1-e^{-(n-m) \beta \omega}\right) f_{F D}(\lambda-\mu-m \omega) f_{F D}(\lambda-\mu-n \omega) \geq 0
\end{aligned}
$$

for $n>m$. From (5.18) we get $J_{\rho_{0}, Q^{p h}}^{p h} \geq 0$.

Remark 5.11 Let us comment the results. If $J_{\rho_{0}, Q^{p h}}^{p h} \geq 0$, then system $\mathcal{S}$ is called light emitting. Similarly, if $J_{\rho_{0}, Q^{p h}}^{p h} \leq 0$, then we call it light absorbing. Of course if $\mathcal{S}$ is light emitting and absorbing, then $J_{\rho_{0}, Q^{p h}}^{p h}=0$.

(i) If $\beta=\infty$, then $\rho^{p h}(m)=\delta_{0 m}, m \in \mathbb{N}_{0}$. Inserting this into 5.16 we get

$$
J_{\rho_{0}, Q^{p h}}^{p h}=\sum_{\substack{n \in \mathbb{N}_{0} \\ \alpha, \varkappa \in\{l, r\}}} n \frac{1}{2 \pi} \int_{\mathbb{R}} f\left(\lambda-\mu_{\alpha}\right) \widetilde{\sigma}_{n_{\varkappa} 0_{\alpha}}^{p h}(\lambda) d \lambda \geq 0
$$

Hence $S$ is light emitting. 
(ii) Let us show $\mathcal{S}$ might be light emitting even if $\beta<\infty$. We consider the case $(E)$ of Proposition 5.3. If $\mathcal{S}$ is time reversible symmetric, then it follows from Corollary 5.10 that the system is light emitting.

If the system $\mathcal{S}$ is time reversible and mirror symmetric, then $J_{\rho_{0}, Q_{\alpha}^{e l}}^{p h}=0, \alpha \in$ $\{l, r\}$, by Corollary 5.6(iii). Since $J_{\rho_{0}, Q^{e l}}^{c}=0$ by Proposition 5.3 we get that $J_{\rho_{0}, Q_{\alpha}^{e l}}^{\mathcal{S}}=0$ but the photon current is larger than zero. So our $J C L$-model is light emitting by a zero total electron current $J_{\rho_{0}, Q_{\alpha}^{e l}}^{\mathcal{S}}$.

Let $v_{r}=0, v_{l}=2$ and $\omega=4$. Hence $\mathcal{S}$ is not mirror symmetric. Then we get from Remark 5.7 (iii) that $J_{\rho_{0}, Q_{l}^{e l}}^{p h}=-J_{\rho_{0}, Q_{r}^{e l}}^{p h} \leq 0$. Hence there is an electron current from the left to the right lead. Notice that by Proposition $5.3 J_{\rho_{0}, Q_{l}^{e l}}^{c}=0$. Hence $J_{\rho_{0}, Q_{l}^{e l}}^{\mathcal{S}} \leq 0$.

(iii) To realize a light absorbing situation we consider the case $(S)$ of Proposition 5.3 and assume that $\mathcal{S}$ is time reversible symmetric. Notice that by Lemma $5.4 s_{c}$ commutes with $\rho^{e l}$. We make the choice

$$
v_{r}=0, \quad v_{l} \geq 4, \quad \omega=v_{l}, \quad \mu_{l}=0, \quad \mu_{r}=\omega=v_{l} .
$$

It turns out that with respect to the representation (5.18) one has only to $m=$ $n-1, \varkappa=r$ and $\alpha=l$. Hence

$$
\begin{aligned}
& J_{\rho_{0}, Q^{p h}}^{p h}=\sum_{n \in \mathbb{N}} \frac{1}{2 \pi} \int_{\mathbb{R}} d \lambda \times \\
& \left(\rho^{p h}(n-1) f(\lambda-(n-1) \omega)-\rho^{p h}(n) f(\lambda-(n+1) \omega)\right) \widehat{\sigma}_{n_{l}(n-1)_{r}}^{p h}(\lambda)
\end{aligned}
$$

Since $f(\lambda)=f_{F D}(\lambda)$ we find

$$
\begin{gathered}
\rho^{p h}(n-1) f(\lambda-(n-1) \omega)-\rho^{p h}(n) f(\lambda-(n+1) \omega)= \\
\rho^{p h}(n-1) f(\lambda-(n-1) \omega) f(\lambda-(n+1) \omega) \times \\
\left(1+e^{\beta(\lambda-(n+1) \omega)}-e^{-\beta \omega}\left(1+e^{\beta(\lambda-\omega(n-1))}\right)\right)
\end{gathered}
$$

or

$$
\begin{aligned}
& \rho^{p h}(n-1) f(\lambda-(n-1) \omega)-\rho^{p h}(n) f(\lambda-(n+1) \omega)= \\
& \quad \rho^{p h}(n-1) f(\lambda-(n-1) \omega) f(\lambda-(n+1) \omega)\left(1-e^{-\beta \omega}\right)\left(1-e^{\beta(\lambda-\omega n)}\right) .
\end{aligned}
$$

Since $\lambda-n \omega \geq 0$ we find $\rho^{p h}(n-1) f(\lambda-(n-1) \omega)-\rho^{p h}(n) f(\lambda-(n+1) \omega) \leq 0$ which yields $J_{\rho_{0}, Q^{p h}}^{p h} \leq 0$.

To calculate $J_{\rho_{0}, Q_{l}^{e l}}^{p h}$ we use formula 5.7). Setting $\alpha=l$ we get $\alpha^{\prime}=r$ which yields

$$
\begin{aligned}
& J_{\rho_{0}, Q_{l}^{e l}}^{p h}=-\sum_{m, n \in \mathbb{N}_{0}} \frac{\mathfrak{e}}{2 \pi} \int_{\mathbb{R}} d \lambda \times \\
& \left(\rho^{p h}(n) f\left(\lambda-\mu_{r}-n \omega\right)-\rho^{p h}(m) f\left(\lambda-\mu_{l}-m \omega\right)\right) \widehat{\sigma}_{n_{l} m_{r}}^{p h}(\lambda),
\end{aligned}
$$


One checks that $\widehat{\sigma}_{0_{l} 0_{r}}^{p h}(\lambda)=0$ and $\widehat{\sigma}_{n_{l} m_{r}}^{p h}(\lambda)=0$ for $m \neq n+1, n \in \mathbb{N}$. Hence

$$
\begin{aligned}
& J_{\rho_{0}, Q_{l}^{e l}}^{p h}=-\sum_{n \in \mathbb{N}} \frac{\mathfrak{e}}{2 \pi} \int_{\mathbb{R}} d \lambda \times \\
& \left(\rho^{p h}(n) f\left(\lambda-\mu_{r}-n \omega\right)-\rho^{p h}(n-1) f\left(\lambda-\mu_{l}-(n+1) \omega\right)\right) \widehat{\sigma}_{n_{l}(n+1)_{r}}^{p h}(\lambda),
\end{aligned}
$$

Since $\mu_{r}=\omega$ and $\mu_{l}=0$ we find

$$
\begin{aligned}
& J_{\rho_{0}, Q_{l}^{e l}}^{p h}=-\sum_{n \in \mathbb{N}} \frac{\mathfrak{e}}{2 \pi} \int_{\mathbb{R}} \times \\
& f(\lambda-(n+1) \omega) \rho^{p h}(n-1)\left(1-e^{-\beta \omega}\right) \hat{\sigma}_{n_{l}(n+1)_{r}}^{p h}(\lambda) d \lambda \leq 0 .
\end{aligned}
$$

Hence there is a current going from the left to right induced by photons. We recall that $J_{\rho_{0}, Q_{l}^{e l}}^{c}=0$.

\section{Acknowledgments}

The first two authors would like to thank the University of Aalborg and the Centre de Physique Théorique - Luminy for hospitality and financial support. In particular, we thank Horia D. Cornean for making us familiar with the $J C L$-model.

\section{References}

[1] W. Aschbacher, V. Jakšić, Y. Pautrat, and C.-A. Pillet. Transport properties of quasi-free fermions. J. Math. Phys., 48(3):032101, 28, 2007.

[2] S. Attal, A. Joye, and C.-A. Pillet, editors. Open quantum systems. I, volume 1880 of Lecture Notes in Mathematics. Springer-Verlag, Berlin, 2006. The Hamiltonian approach, Lecture notes from the Summer School held in Grenoble, June 16-July 4, 2003.

[3] S. Attal, A. Joye, and C.-A. Pillet, editors. Open quantum systems. II, volume 1881 of Lecture Notes in Mathematics. Springer-Verlag, Berlin, 2006. The Markovian approach, Lecture notes from the Summer School held in Grenoble, June 16-July 4, 2003.

[4] S. Attal, A. Joye, and C.-A. Pillet, editors. Open quantum systems. III, volume 1882 of Lecture Notes in Mathematics. Springer-Verlag, Berlin, 2006. Recent developments, Lecture notes from the Summer School held in Grenoble, June 16-July 4, 2003.

[5] M. Baro, H.-Chr. Kaiser, H. Neidhardt, and J. Rehberg. A quantum transmitting Schrödinger-Poisson system. Rev. Math. Phys., 16(3):281-330, 2004. 
[6] H. Baumgärtel and M. Wollenberg. Mathematical scattering theory. AkademieVerlag, Berlin, 1983.

[7] M. Büttiker, Y. Imry, R. Landauer, and S. Pinhas. Generalized many-channel conductance formula with application to small rings. Phys. Rev. B, 31(10):62076215, May 1985.

[8] H. D. Cornean, P. Duclos, G. Nenciu, and R. Purice. Adiabatically switched-on electrical bias and the Landauer-Büttiker formula. J. Math. Phys., 49(10):102106, 20, 2008.

[9] H. D. Cornean, P. Duclos, and R. Purice. Adiabatic non-equilibrium steady states in the partition free approach. Ann. Henri Poincaré, 13(4):827-856, 2012.

[10] H. D. Cornean, C. Gianesello, and V. A. Zagrebnov. A partition-free approach to transient and steady-state charge currents. J. Phys. A, 43(47):474011, 15, 2010.

[11] H. D. Cornean, A. Jensen, and V. Moldoveanu. A rigorous proof of the LandauerBüttiker formula. J. Math. Phys., 46(4):042106, 28, 2005.

[12] H. D. Cornean, A. Jensen, and V. Moldoveanu. The Landauer-Büttiker formula and resonant quantum transport. In Mathematical physics of quantum mechanics, volume 690 of Lecture Notes in Phys., pages 45-53. Springer, Berlin, 2006.

[13] H. D. Cornean, H. Neidhardt, L. Wilhelm, and V. A. Zagrebnov. The Cayley transform applied to non-interacting quantum transport. arXiv, math-ph: 1212.4965v1, 2012. To appear in J. Funct. Anal.

[14] H. D. Cornean, H. Neidhardt, and V. A. Zagrebnov. The effect of time-dependent coupling on non-equilibrium steady states. Ann. Henri Poincaré, 10(1):61-93, 2009.

[15] M. Damak. On the spectral theory of tensor product Hamiltonians. J. Operator Theory, 55(2):253-268, 2006.

[16] C. Gerry and P. Knight. Introductory Quantum Optics. Cambridge University Press, Cambridge, 2005.

[17] T. Kato. Perturbation theory for linear operators. Classics in Mathematics. Springer-Verlag, Berlin, 1995. Reprint of the 1980 edition.

[18] R. Landauer. Spatial Variation of Currents and Fields Due to Localized Scatterers in Metallic Conduction. IBM J. Res. Develop., 1(3):223-231, 1957.

[19] G. Nenciu. Independent electron model for open quantum systems: LandauerBüttiker formula and strict positivity of the entropy production. J. Math. Phys., 48(3):033302, 8, 2007. 